\title{
Alpljabetirdjes Werzęidjuis
}

\section{berjenigen \\ Mitglieder Des Deutidjen Surijtentages}

meldye

Dem Beretn im Iăgre 1908 angehörel.

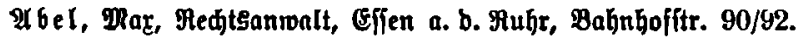

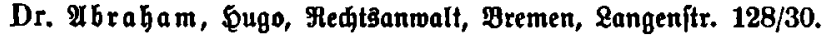

Dr. A brabam, B., Redtsanwalt beim Dberlanbesgeridt Fiel, F̧abritr. 12.

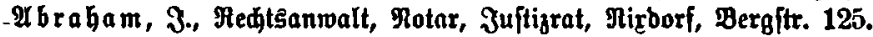

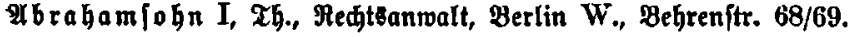

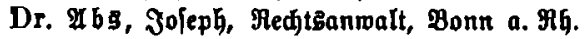

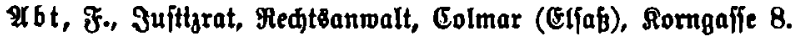

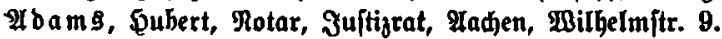

Dr. 2loler, Juftizrat, Cöln a. Mh.

Moler, Bertholb, Eanogeridgtsrat, Saijerstautern.

Dr. van alen, Redttsanmalt, Saargemünb.

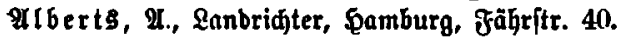

Alberts, Notar, Bemüb a. b. Gifel.

Dr. Arb r edt, Earl, Fechtäanmalt, Samburg, Br. Bāderftr. 15.

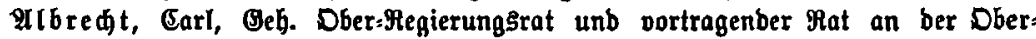
rednunģlammer, Botëbam, Eifenjartítr. 19.

Dr. Alexanber. $\{a$ be, Sugo, Juftijrat, Berlin W., Sanonierftr. 17-20.

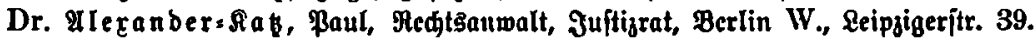

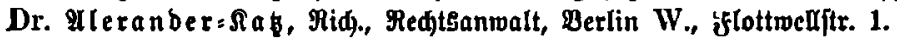

Dr. Allfeld, Bhilipp, tgl. Unid.: \&rofeffor, Erlangen, Biżmardftr. 14.

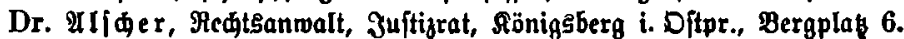

Dr. Als berg, Redtsanwalt, Berlin SW. 68, Bimmeritr. $88 \mathrm{II}$.

Dr. II nbrac, Landgeriftspräfibent, Geh. Dber=3uftizrat, Fiel, Frorftweg 12.

An nores, $\mathscr{A}$ mtstridter, St. Wentel (Bej. Trier).

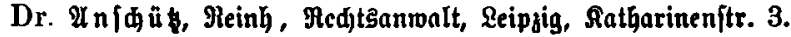

Anspacher, Arv. Liuji, studio, Mailand via St. Antonio.

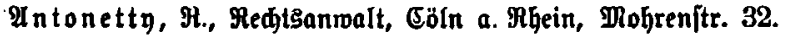

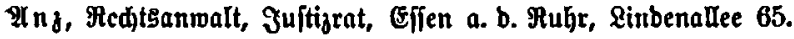

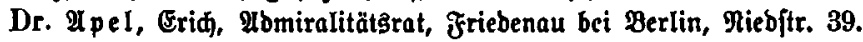

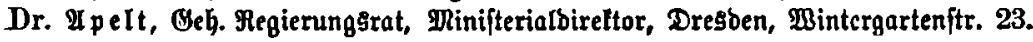

Dr. Ippel, Julius, Beridt3affeffor, Sarlsrube i. B., Raiferftr. 34a.

Dr. Mpt, Dac, Profeffor, Berlin W., Magbeburgerftr. 33.

Dr. Arrnbt, Al, Beh. Mat, Brofeffor, Rönigsberg.

Ilrnot, \&ubwig, Mechtsanwalt, Jujtizrat, ßerlin N., Lothringeritr. 44/45.

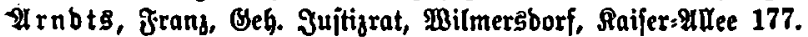


Dr. Ilrnots, Beh. 3ujtijrat, Ecipaig, Stephanitr. 10.

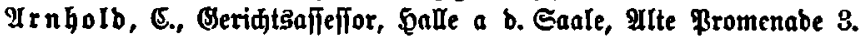

II $\mathrm{nG}$ olf, Mechtsanwalt unb Rotar, Eijitrin.

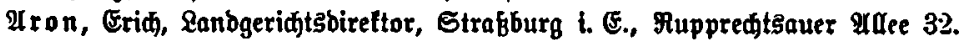

Hronius, Mrthur, Medtzanwalt, Juftizrat, Berlin W., Sdellingitr. 6 I.

Aronfogn, Mectsanmalt, Bromberg.

:Ironfohn, Ald., 3ujtizrat, Bertin W., Leipzigerjtr. 35.

Dr. Il f

Dr. If

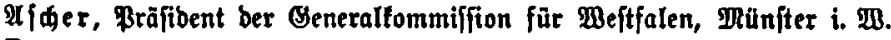

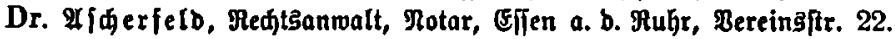

Dr. Af ftrafe 8.

Dr. Ifmann, Arnols Th., Fedtsanwalt, Berlin W. 66, פRauerítr. 2.

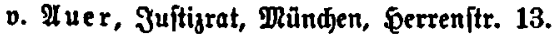

Dr. Aluerbad, Juftizrat, (Jöln a. Mb.

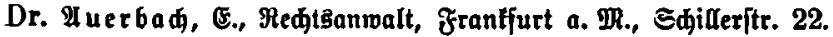

*uguft, פrbert, Fedtsanwalt, St. Sohann a. b. Saar, Mathausplał 5.

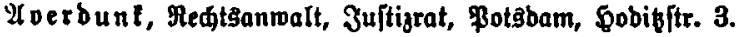

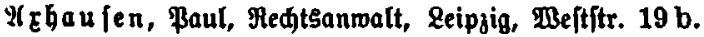

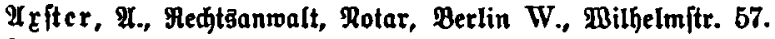

Dr. Ba a f由, Ernft, Bibliothetar ber Siommerj.s:Bibliothet, Samburg, Börfen:2urfaben.

Dr. Bader, Ftedtsanmalt, Amt3ridter a. D.; Stuttgart, \$aulinenitr. 32.

Dr. Badert, Guftav, Medtsanwalt, Mannbeim.

Dr. jur. Babłtübner, Dtto, Randgeridtgrat, Stettin, Raifer Wilgelmitr. 76.

Dr. Ball, Ernit, Redtsanwalt, Berlin W., Botsbameritr. 5n.

Dr. Ballhorn, Friebrid, Redtsanmalt, Berlin N., Chauffeeftr. 130.

Dr. Bamberg, Sugo, Redt5anmalt, Berlin NW. 64, Behrenftr. 22.

Dr. Bandel, Redtsanmalt, Strelno.

Dr. v. Bar, \&., Beb. Sujtijrat, Brof., Ssottingen.

Baebr, Medtsanmalt, Meinbed i. $\$$.

Dr. Baer, $\mathfrak{R}$. S., 3uftizrat, Franffurt a. Thain, Börjenjtr. 15.

Dr. Baer, R., Mechtsanwalt, Coburg, Spitalgafie 4.

Dr. Barban, \$., Redtanmalt, Reipjig, Ricolaiftr. 10.

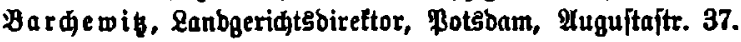

Bartel, Suịtijrat, Saargemün.

Bartning, Adolf, Medttsanwalt, Samburg, Br. Băderftr. 15.

Bartning, B., Amt3riđtcr, Rartsrube i. B., Serrenjtr. 1.

Baerwals, Suftigrat, Bromberg, Theaterplak 3.

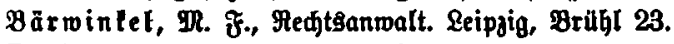

Dr. be 2 arq, Šuftigrat, Frantfurt a. Ilain.

Bafmmann, Meferendar, Bielefelb.

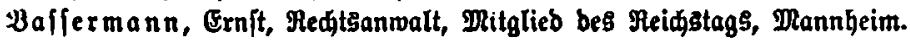

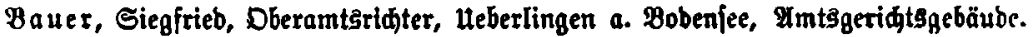

Ba uer, Jatob, FechtBanwalt, Berlin N., Dranienburgeritr. 48/49.

Ha uer, Ehr., Medtsanmalt, Freiburg i. 8 .

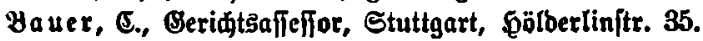

Dr. Bauer, Theobor, Frantfurt a. P., Dodenheimer Lanbftr. 75. 


\section{XVI}

Dr. Baumert, Medstganwalt, Rotar, Juftiłrat, Spanbau, Potsbameritr.

Baumgartner, Staatsanwalt, Sartsruthe $i .: B$.

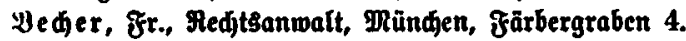

Deder, \&ubwig, Stabtrectitrat, Sarlöruhe i. 9 .

Dr. Beđerer, B., Hechtsanwalt, Berlin W., Dohrenftr. 13/14.

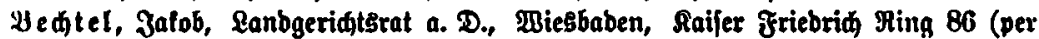
Ior. Dberitleutnant a. D. Th. v. Both).

1). Beder, Mobert, Meditzanwalt, 3ujtizrat, Düffelborf.

- Wider, Beorg, Meditzanmalt, Bertin 0., Brōie Grantfurteritr. 102.

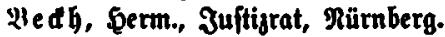

Dr. jur. Bedmann, Bilhelm, Lanbridtex, Dortinüb; Mmbtift. 68.

Dr. Beer, E., Mechtsanwalt, Berlin W., Bebrenjtr. 24.

Dr. Beer, Max, Medtsanmalt, Rotar, 3uftizrat, Berlins Steglił, Itbredts ftrafie 12.

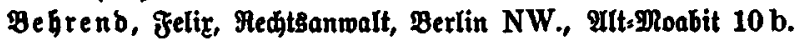

Dr. Befrenb, Midjarb, Megierungstat, Berlin 2 ., Biegenburgeritr. 1.

Bebrendt, Severin, Medtsamwalt, Berlin W., Botsbameritt. 19 I.

Beier, Eanbgeridatsbireftor, Breslau, Boetheitr. 16.

Dr. Bet finger, \$ugo, Rarlstube 1. 8., Raiferitr. 105.

Dr. Bendifer, Lanbgeridtstat, Sartsrube i. $\mathfrak{B}$.

Benber, Medtsanwalt, SarlEruke t. B., Saifer[tr. 197.

Benber, Notar, Sarlsrube i. 8 .

Benfen III, Fedtsanwalt, Sannover:

Dr. Berbig, Dr., Fedttanwalt, Berlin W., Friebridftr. 78.

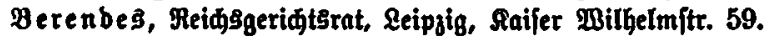

Ferger, Julius, Эuftizrat, Seipjig, Micolaiftr. 6.

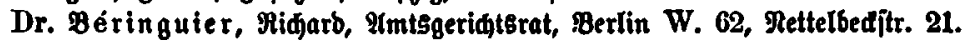

Berlin, Max, Dberlanbeşgeridtsrat, Rümberg, Frauentorpla 37.

Dr. Bernbt, Redtsanwalt, Stettin, Breiteftr. 6 I.

Dr. Berngeim, Seinrid, Redtsanwalt, Mhannbeim.

Werni, Ober=2lmtEridter, Sarlsrube i. $\mathscr{B}$., Sarlftr. 66.

Bernftein, 5., Suftizrat, Berlin N., Dranienburgerftr. 1.

Dr. Bernftein, Milh., 3uftigrat, Berlin NW., Dorotheenjtr. 65/66.

Dr. Befeler, 3uftizminifter, Berlin W., Milfelmitr. 65.

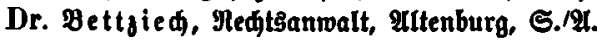

Dr. Beyerle, Conrab, Brofeffor, Böttingen.

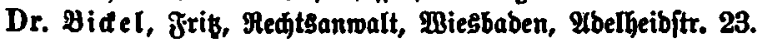

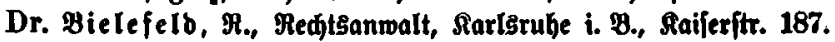

Bielf

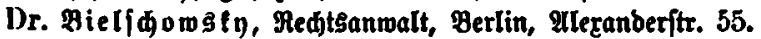

D. Dr. Bierling, Beh). Suftizrat, Profeffor, Oreifŝwalb.

Dr. Biermann, Brofeffor, Biefen.

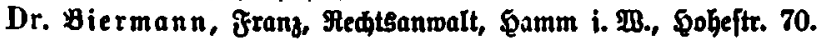

Dr. Binber, 3., Brofeffor, Erlangen.

Dr. Binbing, Sart, Bebeimrat, Brofeffor, Seipzig, Bismardftr. 6.

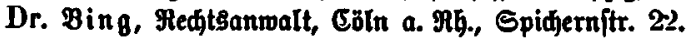

SHing, Dhax, Redtäanwalt, MRünden, Thal 67 II.

Dr. Binswanger, Pedtisanwalt, Jultizrat, Fugşurg, Bottgartftr. 6. 


\section{XVII}

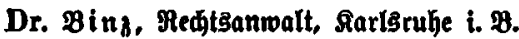

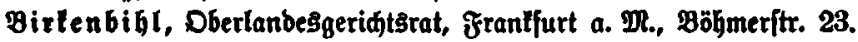

Dr. Birtmener, Beh. \$ofrut, Brofefjor, פeündjen, Diterwalbftr. 95.

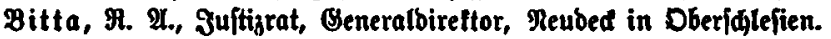

Dr. Bitter, Fedtsanwalt, Rotar, Fiel.

gittner, Amtstiditer, Dramburg.

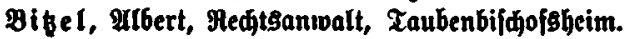

Blandmeifter, Lanbgeridtşbireltor, Berlin NW. 21, Zurmftr. 4.

Blafdfauer, H., Reditsanwalt, Berlin SW., Rommanbantenitr. 7/8.

Dr. Bleidjer, Stantsanmalt, Farlşuthe i. 8 .

Dr. :Iod, Arthur, Medtsanmalt, Bforjheim.

Dr. Blodi I, Reditsanmalt, Mündjen, Briennerítr. 4.

Dr. $\mathfrak{B l}$ l m, :., Mechtsanwalt, Mannநeim C. 33.

BI I me, Reidjgeridtstat, Leipgig, Satobitr. 5.

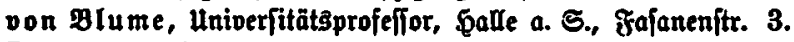

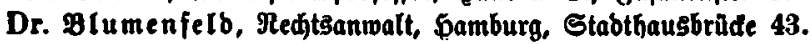

Bobreder, Redttsantwalt, Berlin C., Bontarbitr. 4.

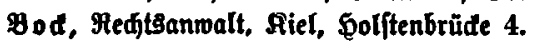

Pod, Mechtsanwalt, Magbeburg.

Bocdh, DR., Redtsanwalt, Rartsrube i. $\mathfrak{B}$.

Dr. Boedel, Friz, Redtsanmalt, Zena.

Bodelmann, Suftizrat, Riel, Solftenitr. 9.

Dr. Bobenftein, Regierungsafieffor, Effen=Яubr, griebridjitr. 2.

Dr. Bogen g, (S. A. E., Betlin W., DRartin Rutherftr. 74.

Bohm, Imtsgeridtstrat, Sannover, Đorffitr. 5.

Boebm, Redtęanwalt, Berlin W., Röthener[tr. 45.

Bo ju n ga , Seh. Sujtijrat, Medjtsanwalt, Motar, Sannover.

Dr. Bonbi, Jelix, Suftizrat, Reditsanmalt, Rotar, Dresben, Sohann Beorgens Ituee 9.

Bonné, C., Recttsanwalt, BabensBaben, Bernsbaderftr. 32.

I)r. von goenningbaufen, Mledtsanwalt, \$amburg.

Bongel, Meditsanmalt, Rotar, Dortmunb, Ofterheđrweg 61.

Borders, Mb., Beheimer 3uftizrat, Regtênmalt, Celle.

Dr. Borinsti, Redtsanmalt, Berlin W., Drohrenftr. 16.

Dr. Bornhat, Conrad, Profeffor, Berlin S.W., Blüderplał 2.

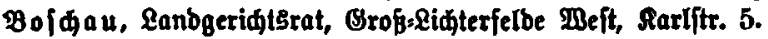

Dr. Boetbfe, Sammergeridtstat, Berlin W., In ber Mpofteltirdje 1.

Dr. Bovenfie jen, 21mtstidter, \&öbau \$.»r.

Boyens, Juftizrat, Reipzig.

Boylen, Imtsridter, Samburg 26, Mittelitr. 70.

Dr. Bradenboeft, Eb., Medtteanwalt, Đamburg, Or. Blciøen 5.

Dr. Bradenhoeft, Detavio, Redtsanwalt, Samburg, Gr. Bleiden 5.

Branbau, Meferenbar, Siel.

Branbes, Bürgermeifter, Glüditabt.

Branbt, Suftizrat, Redtకanwalt, Rotar, Siel, Solftenftr. 43, I.

Brauer, Juftizrat, Meditsanwalt, Rotar, Cofel i. Ob.:Sdjl.

Dr. Bra un, Beheimer 3ujtizrat, Medttanwalt, Berlin W., Behrenjtr. 3.

Braun, P., Redtsanwalt, Mebs, Madtftr. 
Dr. Breit, SameB, Redtsanwait, Dresben, Elifenftr. 10.

Brenten, SenatSpräfibent, \$amm i. $\mathfrak{B}$.

Brenneđ̛e, Carl, Senatşprăfibent, Berlin N.W., Caloinitr. 8, III.

BresIaucr, Bernharb, 3uftizeat, Berlin C., Saifer BillyeIm/tr. 3.

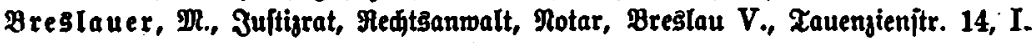

Dr. Brint, L., Notar, Mayen i. Mhlb.

Dr. Brike, Redtsanwalt, Bwidau i. S, Dhorik Srabenweg 7.

Dr. Brod, Medteanwalt, Berlin N., Ehauffeeftr. 123.

Dr. Brobni Georg, Brivatbojent, Salle a. S., Reidjarbftr. 17.

Dr. Brobnib, Redtanmalt, Berlin S.W., Jimmeritr. 21.

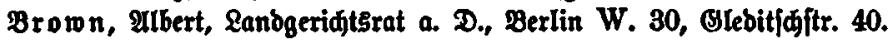

Brud, Beorg, Medtsanmalt. Berlin W., MRaueritr. 68.

Br ü đelmeier, Redtsanwalt, Reipjig.

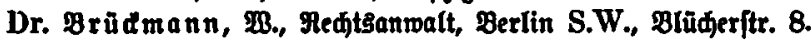

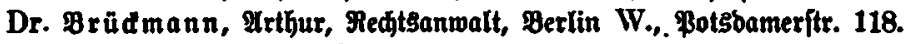

Br ühl, 3uftigrat, Berfin C., Burgitr. 28.

Brunf, Fectsanmalt, Subwigbbafer.

Dr. Brunner, Beh. Juftigrat, Profeffor, Berlin W. 62, \&utherftr. 36.

Brunstwis, S., Rechtsanmalt, Notar, Reuftreli.

Dr. Brunswig, Peter, 2lffeffor, Ditarbeiter im Sefretariat ber Deutidien Banf, Jriebenau, Mielandftr. 6 .

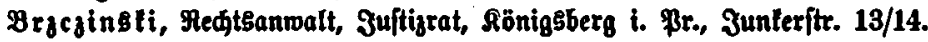

Pud, Oef. Dberoभiegierungstat, SarlBrulje i. 2 .

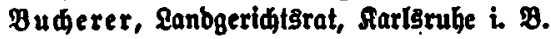

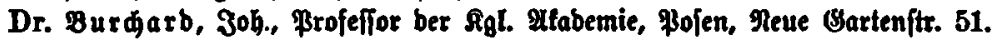

Burbad, MRar, Dberlanbesgeridtsrat, Röniggberg i. Pr., Sintertragheim 17.

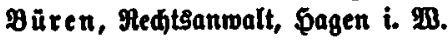

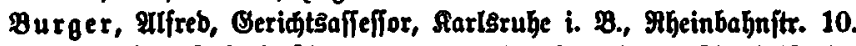

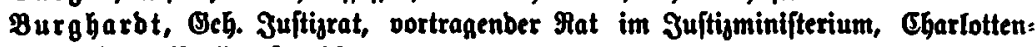
burg, Perlineritr. 23.

Burbheim, Maul, Medtsanmalt, Rotar, Sultigrat, Minben (Meftfalen).

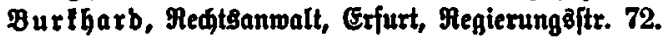

Dr. Bu\{d, Ebuarb, 3uftifrat, PR..@labbad.

Bufd, Rouis, Feidjggeridtsrat, Reipjig.

Dr. Büttner, Megierunggrat, Pot3bam, Reue Rönigitr. 21.

Dr. But, Th., Redtsanwalt, Rartsrube i. $\mathscr{D}$.

Butgengeiger, Eanggeridtsrat, Sartsrube i. $\mathscr{8}$.

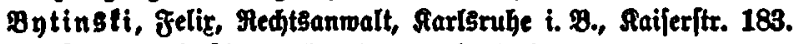

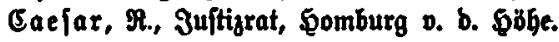

Caben I, QYlbert, Redtteanwalt, Eäln, Rattenbug 19.

Caben, \&ubwig, Redtsanwalt, Cöln a. Rh., Elijenftr. 18.

Dr. Eahn, Beh. Regationsrat, Berlin W. 12, Daienjtr. 5.

Dr. van Ealter, Profeffor, Strajburg i. C.

Dr. van Caltes, Brofeffor, Biejen.

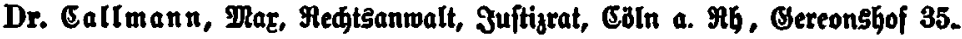

Dr. Eannebt, Earl, Redtsanwalt, Motar, Berlin W., Maueritr. 41.

Dr. Eantor, D., Medtsanwalt, Sarlsrube i. $\boldsymbol{B}$.

Caro, Midarb, Redjtsanwalt, Berlin C., Stralaueritr. 3/6. 


\section{XIX}

Cariten 9, Reditsanwalt, Rotar, Cottbus, Bahnhofftr. 19.

CGobzieBner, \&., Redtsanwalt, Berlin C., Saifer Bilhelmftr. 49.

CGobjiesner, Max, Fedtsanmalt, Berlin W., unter ben Sinben 42.

Ehobjiesner, Siegfrieb, Medttsanwalt, Charlottenburg, Berlinerftr. 121, I.

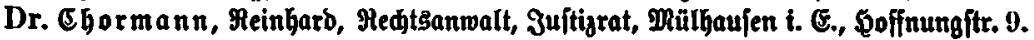

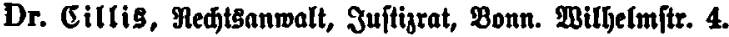

CI a ajen, Beh. Regierungstat und vortragender Rat in ber Dberredinungstammer Potabam, 3äger=\{lltee 16.

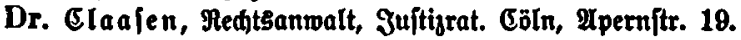

Dr. Clauโen, Amteridter, Reumüniter.

Dr. Clemen, C., Rectsanwalt, Dannheim B 2. 10 a.

Dr. Cohen, Dtto, Dberlanbeşgeriđtstrat, \$amburg, Leinpfab 66.

Dr. Cohen, Dax, Medtsanwalt, நamburg. Stabthausbrüde 43, I.

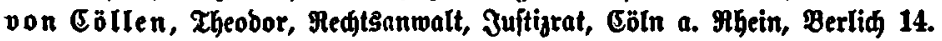

Dr. Co hn, Dartin, Reditsanwalt, Berlin W., Corneliusitr. 5 .

Cohn I, 3uftijrat, Berlin W., Sägerftr. 20.

Dr. Cobn, \&ubmig, Beheimer 3ultigrat, Brestau, Âgnesftr. 1, П.

Cohn, Jerbinand, Redițanmalt, Rotar, Juftizrat, Ologau, Millelmsplaßs 8.

Cohn, Âmtsgeridjt3rat, Breslau, Boetheitr. 16, II.

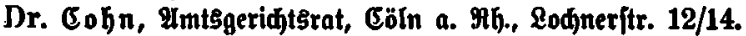

Dr. Cohn, Eeorg, Brofeffor, Bürid V, Earmenftr. 18.

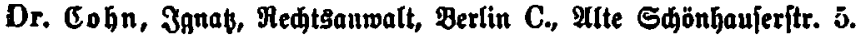

Cohn, Louis, Medtsanwalt, Notar, 3uftizrat, Berlin N., Brunnenitr. 25.

Dr. Cohn, Dstar, Redtsanmalt, Berlin C. 25, Ranbsbergerfitr. 58.

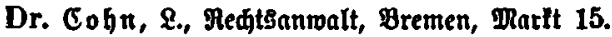

Dr. CoGn, Medtiganwalt, Defíau, Berbfterftr. 56.

Coljn, Beorg, Meditsanmalt, Rotar, Jujtijrat, Tilfit.

Cobn, Eugen, Redttanmalt, Charlottenburg, Fantfit. 123.

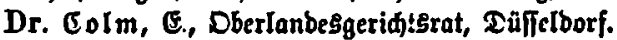

Corell, 2llbert, $\Re_{g} l$. Ianbgeridtstat, Midjaffenburg.

Dr. Cramer, Fedtsanwalt, Bielefelb, Bütersిloheritr. 9a.

Cremer, Dtto, Amtsridter, Duisburg, frriebrid Milgeluitr. 23.

Crome, Baul, Medtsanwalt, 3uftizrat, Berlin S.W., Belfesquianceftr. 91.

Dr. Crome, Carl, Brofeffor, Bonn, Saijerftr. 57.

Dr. Crufen, Saiferl. Oberrifter, Tingtau (Siautfjau).

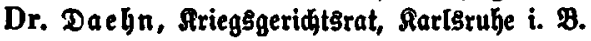

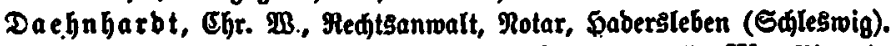

Danielcil, Sammergeriøtşrat, Geh. Suitizrat, Berlin W., Rictoria Louijena plał $1, I$.

Danziger, Max, Medtsanwalt, Berlin C., Alleganberfitr. 71.

Dr. Danjiger I, $R$, Medtsanwalt, Betlin C., Molfenmartt 12/13.

Danjiger, Medtsanwalt, Motar, Brieg (Bej. Brcslau).

Dr. Darmfta ebter, Medtsanwalt, Mannfeim.

Daus, 3., 3uftizrat, Ultona (Elbe).

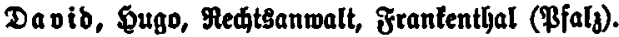

Dr. Da oibjobn, Reo, Reditsanwalt, Berlin N., Ehaufieeitr. 8.

Davibjohn, Willy, Medtsanwalt, Silbesheim.

von ber Deden, Senatspräfibent, நamm i. 2 . 
DeboIph, Juftigrat, Redtsanwalt, Plotar, Cottbus.

Dr. Degentolb, Seg. Gofrat, Profeffor, Seipjig, Bismarditr. 19.

Dr. Dei $\bar{f}$, 3uftizrat, Leipjin, Bismatditr. 2.

Dr. Delaquis, E., BerlinsWilmersborf, Saijersथlee 19.

Delbrũd, Beh. Dberregierungstrat, Br.sididerfelbe, Sternftr. 15.

Dr. Delius, Sammergeridgtšrat, Berlin, Sdaperftr. 32.

Dr. Demler, Rgl. Notax, Lauf a. b. Megnip.

Dr. Denefe, Medttganwalt, Göttingen.

Dr. Detmolb, Brofeffor, Ubttingen.

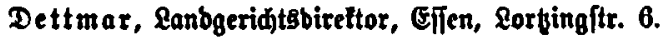

Dr. Deut|ळ, Redtsantwalt, DRannheim.

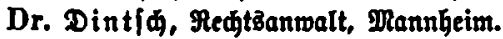

Dr. Didel, Sarl, Oeriøtstrat, Brofeffor, Charlottenburg, Snejebeditr. 22.

Dr. Didel, Sammerrat, Merniperobe.

Dr. Diebolb, Erwin, Seridtsaffeffor, Sarlsarube i. B., Sophicnitr. 11.

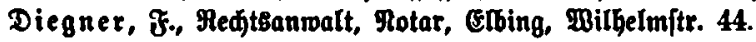

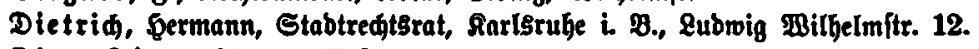

Dieb, Ariegşgeridt3rat, Majtatt.

Dię, Reidsanmalt, Reipjig, Raijer Milhelmftr. 64.

Dr. Die \$, E., Redtsanwalt, Sarlstuhe i. $B$.

Die

Dinfgrae ve, Redtsanwalt, Juftizrat, OHurid i. Ditfrieslanb.

Dietrid, Mteftęanwalt, Sultizrat, Prenglau.

Dr. Dittenberger. Geinrid, Redtsanwalt, \$alle a. S., Tiergartenftr. 5.

Dr. jur. et phil. Dodow, Granz, Pribatbozent ber Medte, Đeibelberg.

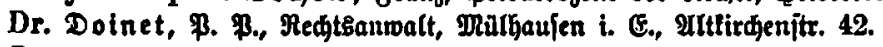

Dr. Doelter, Atuguft, Dberlanbesgeridterat, Rarlšrube i. B., Selmbolj: fitrafie 13.

Donath, Maul, Redtsanwalt, Rotar, Sdgeibnit.

Donnevert, IRar, Reditsanwalt, Meţ, Bantitr. 7.

Dorenborf, Juftizrat, Magbeburg.

Doermer, Redtsanwalt, Elberfelb.

Dr. Dormituer I, S., Redtsanwalt, Mürnberg.

Dr. Dorner, E., Ranbgeridtspräfibent. Sarlsrube i. B., Meifitab̆ftr. 4.

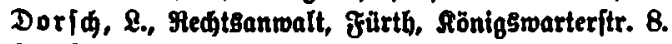

Dorft, Fran, Suftizrat, Rotar, Söln a. Mhein, Rorbertitr. 26.

Dove, Đeintid, Lanbgeridtsrat a. D., Berlin W., friebrid Milgeimitr. 10.

Dreidex, Carl, Medtsanwalt, Sdpéinfurt.

DreweB, ImtBridter, Stettin, QRoltfeftr. 19.

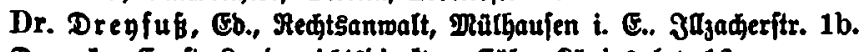

Dronfe, Ernft, Lanbgeridtsbirettor, Eöln, Sönigsplał 16.

Druder, Medtsanwalt, Reipjig.

Dr. Druder, Rartin, RedtsanwaIt, Seipjig, Mitterftr. 1 II.

Dr. Dührenbeimer, Medtbanwalt, Manngeim B 4, 1.

Duffner, Dber:Staatsanwalt, SartSrube i. 8 .

DümIing, P., Fedttsanwalt, Durlad.

Dũ mmler, 3ujtizrat, Diülbaulen i. E.

Dantels büblex, Rlexanber, Redjtsanwalt, Mündjen, Dienerftr. 10, II. 


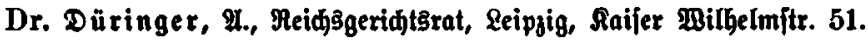

Dürr, Lanogeridtsbirettor, Sartsruhe i. 2 ., Sofienitr. 18.

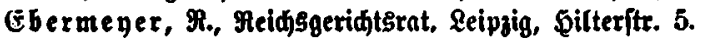

Dr. Eberítabt, Geridt\&affefior, Frantfurt a. 9 ., Squbertitr. 10.

Edert, \&., Lanbgeridtsrat, Oppeln, Molenbergeritr. 15.

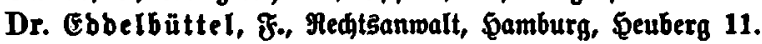

Dr. Eber, Carl, Medtsanmalt, Mannheim.

Edjarb, C., Medtsanwalt, Motax, Bremen, Dbernitr. 22/24.

Dr. Eger, Medtsanmalt, Berlin W. 8, Martgrafenjtr. 24.

Dr. EGlers, Fedttanmalt, Riel, Befeler gllee 31.

Dr. Eלrenberg, Univerfitätsprofeffor, B̧öttingen.

Ehrlid, Max, Jujtizrat, Berlin W., Taubenitr. 16/18.

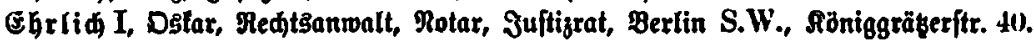

Dr. Ehrlid, Medtsanwalt, Xilfit.

Ëiđel

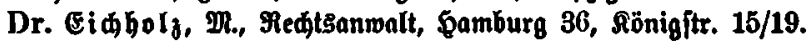

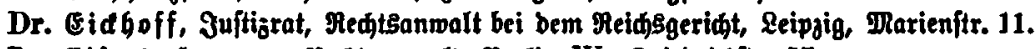

Dr. Eifert, Germann, Medtzanwalt, Berlin W., Friebridftr. 57.

Eifele, 2., Redtsanwalt, Ellvangen.

Dr. Eifemann, Mac, MedtEanwalt, Rürnberg.

Eifenmann, Suftigrat, Berlin N., Chauffeeftr. 2,

Ei fenftäbt, Redttsanmalt, Berlin S.W. 48, Milhelmitr. 20.

Dr. Eifenftäbt, Ilffreb, Mecttanwalt, Berlin S.W., Bimmerftr. 16/17.

Eifeng immer, H., Medtsanmalt, Itülhaufen i. E., Soffinungftr. 17.

Eibel, Redtşanwalt, Rarişrulje i. B., Raijerftr. 66.

Elfiner, Stabtređttôrat, Baben,\$aben.

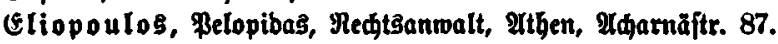

Dr. Ellenbogen, Medtsanwalt, Rarls̈rube i. 2., Raiferitr. 94.

Dr. Eller, Lanogeridtsbirettor, Sarlšrube i. $\mathfrak{B}$.

Dr. Ell ítätter, CarI, Juftitiar, Beutgen.

Dr. EIfaffer, Senatgpräjibent, Stuttgart, Olgajtr. 6.

Elsbađ, Redtsanwalt, Berlin W., MRohrenjtr. 6.

Elfperger, 2 ., Eanbgeridgtêprăftbent a. $D$, \$of in Bayern.

Dr. El bbađer, \$aul, Brofeffor, Charlottenburg, Dernburgplaß 1.

Elge, Curt, Juftigrat, \$alle a. b. Saale.

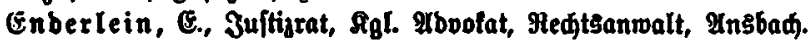

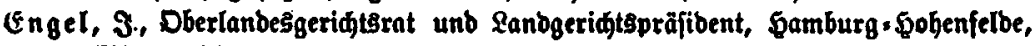
Eilenau 34.

Dr. Engelbarbt, M., Oberamtiridter, Aarlärube i. $\mathfrak{B}$.

Dr. Engelmann, R., Staatsanwalt, PRünden.

Dr. Engler, R., Oberamterrifter, Ettlingen.

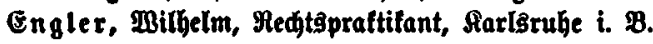

Dr. Enneccerus, Geh. Juftijrat, Profeffor, Direftor bes juriftijden Seminars, PRarburg i. $\mathfrak{Q}$.

Dr. Enneceerus, @eh. \$uftizrat, \$rofeffor, Marburg i $\$$.

Dr. Enzmann, Dtto, Dber.Jujtizrat, Ehemnity i. S., Moímartt 4, I.

Dr. Ephraim, Drartin, Mectsanmalt, Frantfurt am Diain.

Dr. Epftein, Emil, Medtsanwalt, Iugşurg. 


\section{XXII}

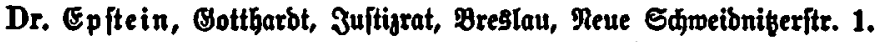

Eridfen, Sophus, Redtsanwalt, Rotar, Gabersleben i. Saleswig.

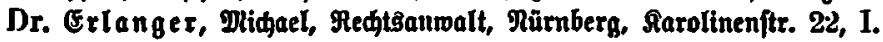

Dr. Erlanger, Fedtsanwalt, Stuttgart, Tubingerftr. 14A 1.

Erythropel, Gebeimer Suftigrat, Leipjig.

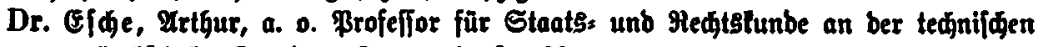
நodjహule, Dresben, Rommeniusftr. 22.

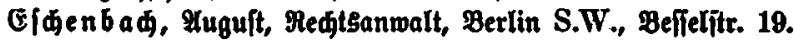

Effer, Mobert, Bebeimer Juftizrat, CöIn a. Rh., am \$of 20.

Dr. Etler, Prthur, Medtsanwalt, Mottweil.

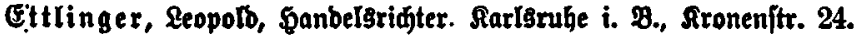

Dr. Euli $\$$, Max, Meditsanwalt, DreB̉ben, Mingitr. 25.

Dr. Eyd, Erid, CharLottenburg, פilmersborferftr. 8a.

Dr. Faber, \&anbridter, Stuttgart, IItezanberjtr. 17.

Dr. FaGle, Redtsanwalt, MRünter i. 2 ., Mlter Steinweg 5.

Fable, 3ujtizrat, ßojen.

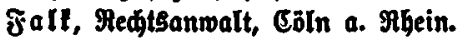

Faltenbad, Suftigrat, PRerjig.

Dr. Zaftenratb, §ofrat, Cöln a. Mb., Cbriftophftr. 12.

Dr. Fedt, Sermann, Megierungsaffeffor, Rarlsruhe i. M., Mathyftr. 8.

Feildenfelb I, Daniel, Meditsanwalt, Berlin W., gRartgrafenitr. 62.

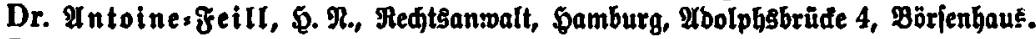

Dr. Fetfenberger, Staatsanwalt, Magbeburg, Raifer Dtto Ming 34.

Dr. Fet \$t, \$alther, Medttanwalt, Mannheim.

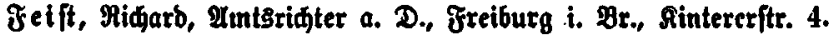

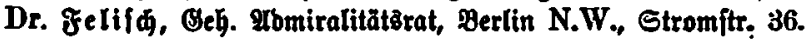

Fel , Faver, Meferenbar, Beberidweier b. Colmar i. E.

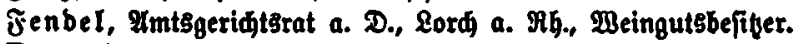

Dr. Fefter, Ibolf, MedtEanwalt, Rotar, 3uftizrat, Frantfurt a. PR., \$odftr. 29.

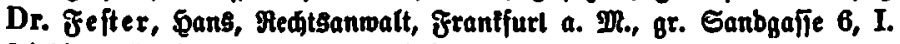

Fidter, 5., Ymtgridjter, Dueblinburg.

F̧ iebler, ש., Medtsanwalt, Rotar, Rehe, Safenftr. 114.

Dr. Finger, Iluguft, univerfitätš Brofeffor, Đalle a. S., MRagbeburgerftr. 26.

Dr. Finjenhagen, Ymtsridter, Rtel, Droltteftr. 11.

Dr. Fifajer, Dtto, Geheimer \$uftizrat, Dberlande\&geridtSrat, Profeffor, Breslau, Mongauptftr. 3.

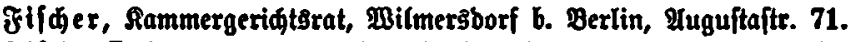

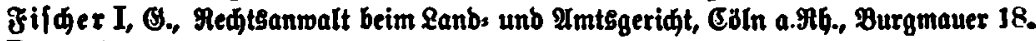

Dr. Fif

Dr. Fifäer, \$anz, atbredt, univ.sprofeffor, Moftod i. $\mathbb{R}$.

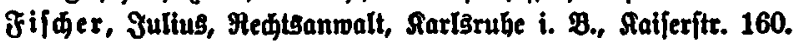

Fiła

Dr. Fibler, Sammergeridtstat, Charlottenburg, Sdlüteritr. 31.

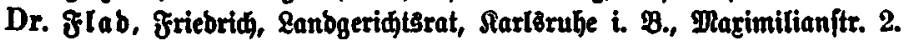

Dr. \&lata u, \&ubwig, Medtsanwalt, Berlin N.W., \&riebriditr. 167.

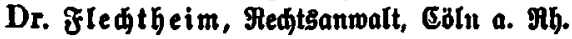

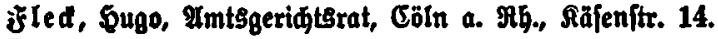

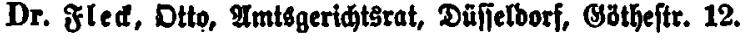




\section{XXIII}

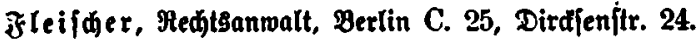

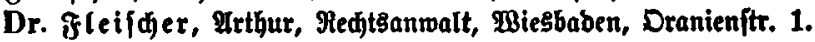

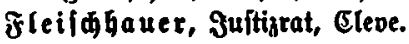

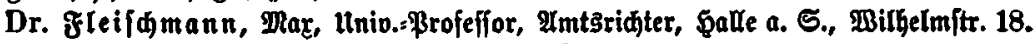

Flie B, Redtsanmalt, Rotar, Juftizrat, Fraujtabt.

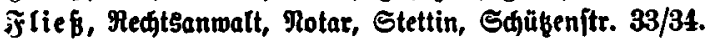

Dr. Fontheim, Beorg, Referenbar, Berlin, Ranteftr. 4.

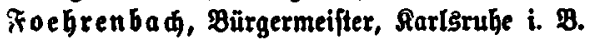

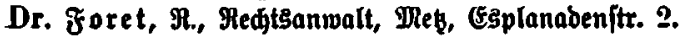

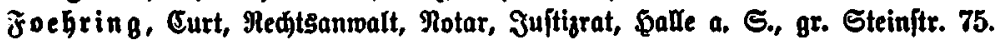

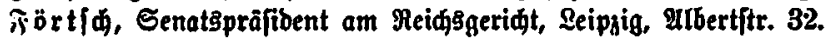

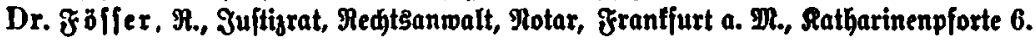

Dr. Frant, Meint., Profeffor, Zübingen.

Frant, Ebuarb, MechtEanwalt, Fonjtanz. Mosgartenftr.

Dr. Franf, Subrig, Mectsanmalt, Pannheim.

Jrande, $\mathfrak{B}$. Chr., Dberlanbeşgeriđjtకarat a. D., \annoder, Böbederftr. 10.

Dr. Fraentel, Gerbert, Itchtsanwalt, Berlin C., Dirtienftr. 46.

Dr. Fraentel, \$alter, Redtsanmalt, Berlin C., Gertraubtenftr. 18/19.

Zranfenburger I, \&., Redtsanwalt, Rürnberg. Carolinenitr. 1.

Franzen, E., Medtsanwalt, Notar, Juftigrat, Riel, Zange Meibe 11.

Dr. Freefe, 1. Staatsanwalt, Rorbbaulen, Stollbergerftr. 11.

Srege, Beh. Suftizrat, Siegnik, Sdjeibeftr. 15.

Frentel, Paul, Jujtizrat, Reipzig, Satharinenitr. 29.

Dr. Freubenthal, Profefior, Grantfurt a. $\mathfrak{R}$.

Freunb, Rubwig, Nechtsanwalt, Nannheim.

Frey, Rectisanmalt, Rarlšrube i. B., BaIbitr. 8.

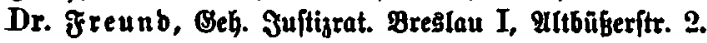

Freyta g, Otto, Emil, Reditsanwalt, Reipzig, Ricolaiftr. 17.

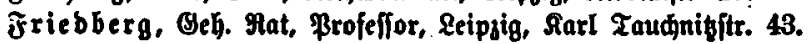

î́ riebberg, Eanbgeridłt3präfibent, Sannover.

Dr. Friebberg, \&., Medtsanmalt, SarlSrube t. $:$.

Dr. Friebe, \$ugo, Redtsanwalt, Samburg, Sr. Theateritr. 12.

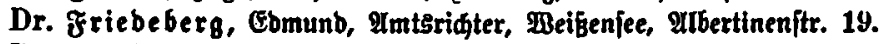

Dr. Friebemann, Ebmunb, Suftijrat, Berlin W., PotBbamerftr. 12.

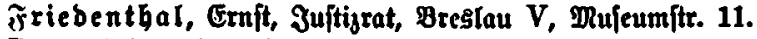

Dr. Friebenthal, Felix, gechtsanwalt, Berlin W., \&übowitr. 48.

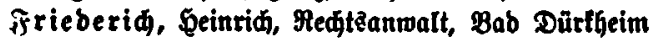

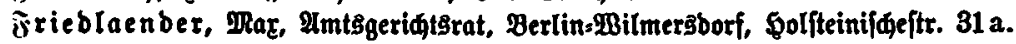

Dr. Friebla enber, Georg, Bantbireftor, Suftizrat, Berlin W., Wilfelmitr. 71.

Dr. Frieblaenber, Fectisanmalt, Rotar, Sultijrat. \$otsbam.

Dr. Frieblaenber, Carl. Eriđ, Mectianwalt, Berlin W., Rronenftr. 3.

Frieblaenber, Fr., \$uftizrat, Berlin W., \&ütonftr. 46.

Frieblaender, \&eo, Redtsanwalt, Berlin W., Sodjitr. 60.

Dr. Frieblaender, Redtianwalt. Rotar, Rublinis Ob.s Sdl.

Dr. Frieblaenber, MRax, Medtşanwalt. MRünden, Meuhaujeritt. 14/II.

Dr. Friebleben, Frib, Juftigrat, Frantfurt. a. $\mathbb{1 R}$.

Dr. Friebmann, Th., Juftijtat, Berlin N.W., IIt=פroabit 119.

Friebmann, Suftizrat, Ologau, Wilgelmplaţ. 


\section{XXIV}

Dr. Friebmann, Fedtsanwalt, Sarlsrube i. :B., Raijeritr. 141.

Dr. jur. Friebmanu, Ilffeb, Berlin W. 10, Xiergartenftr. 8.

Friebmann, \&eonbarb, Medtsanwalt, Berlin W. 8, Rronenitr. 64.

Dr. jur. Friebrids, Carl, Redttsanwalt, Düffelborf, Bismardftr. 84.

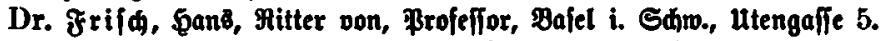

Friłe, Beh. Ober:Suftizrat, Berlin W. 62, Bormjeritr. 11.

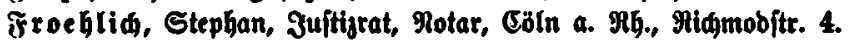

Fromher, Beter, Ranbgeridtstat, MRosbad.

Fromm, Bruno, Rammergeridtsrat, Beh. Şuftizrat, Berlin W., Maajenfitr. 14.

Fromme, D., 3uftigrat, Walberftabt.

Dr. Frommbolb, Beorg, Brofeflor, Greifşmalb.

Frormann, FmtBridter, Flensburg, am Burgfrieb 7, I.

Frowein, Oberverwaltungsgeridtgrat, Berlin W., Surfüritendamm 20.

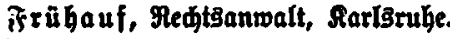

Dr. ₹̛ud9, Cugen, 3uj̣tijrat, Berlin W., Potgbameritr. 39 a.

Fuめs, E., Redtsanwalt, Berlin W., Franzöfifdie Str. 13.

FFuds I, Dlax, 3uftizrat, Berlin W. 9, Botsbameritr. 13.

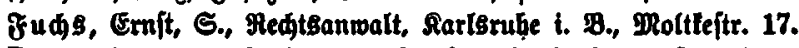

Dr. F̈ubrmann, \&anbridter, Greifswafb, Steinlederftr. 40.

Dr. \&ulb, \&., Rechtsanwalt, Suftizrat, MRaing, Babnhofitr. 6.

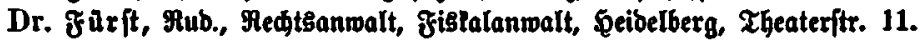

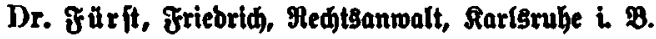

(S) a bel, E., Redtsanmalt, Notar, Sqneibemühl.

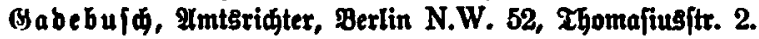

Dr. Ba ibfd, Redtsanwalt, Rotax, Chemnit (Sadjen) Theateritr. 84.

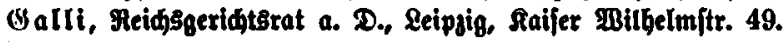

Dr. Ballinger, Redtsanwalt, Rürnberg.

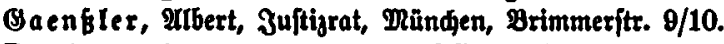

Dr. Bans, S., Redtsanwalt, Profeffor, Leipjig, Bismardítr. 12.

BaettenB, M., AmtBgeridtsrat, Moftod i. $\mathfrak{T}$.

Gebharbt, MedtEanwalt, Motar, 3uftijzat, Frantfurt a. D.

Gebeftiftung, Dresben, Rleine Brubergaffe 21.

Dr. Bebrte, Max, Meditsanwalt, Frantiurt a. PR., Sdjillerfit. 5.

Dr. Beier 8 befer, Fedgtsanwalt, Nürnberg.

Dr. Beiger, IIffreb, Meditganwalt, frantfurt a. MR, Sdjillerftr. 22.

Dr. Beiger, Bertjolb, Jujtigrat, Frantfurt a. MR., Bdilleritr. 2\%.

Beiler, R., MedjtBanwalt, Mannheim.

Geifier, Jujtigrat, Freiberq i. S.

(S) ntil, 3., Mect5anwalt, Mannheim.

Dr. Berbarb, Redtsanwalt, Mannbeim.

Berharbt, Stephan, 3uftijrat, Berlin W., Wilhelmftr. 90.

Dr. Berlanb, Brivatbogent, Beridtsaffefior, Sena.

Befelbradt, Medtsanwalt, Rotar, 3uftijrat, Dortmunb.

Dr. Gerfon, Redtsanwalt, 3uftizrat, Berlin W., Friebridfitr. 76.

(B) effele, D., Redtsanwalt, Memmingen.

Biebne. \&anbreridgterat, Sartsrube i. B.

Dr. Bierle, Dtto, Beh. Juftizrat, Brofeffor, Ebarlottenburg, Carmerftr. 12.

Dr. Orierle, Sulius, o. Profeffor, Aönigsbergs:Dittel'bufen, Souijenallee 16. 
(Si) ie de, Sujtizrat, Magbeburg, Breitemeg 230.

Glafer, Suftizrat, Breglau.

(SIa smader, \$., Motar, Mombady i. Sothringen.

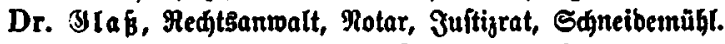

Glogomsti, Redtsanwalt, 3uftizrat, Rawitfd.

Dr. \& I ü S mann, Mlf., Stabtrat, Mixborf, Sontasftr. 66.

(8) mür, Di., Projeffor, Bern i. @dipeiz.

(3) oite in, Medtsanmalt, Mannheim.

Solbberg, Suftigrat, Medtsanwalt, Rotar, Marburg a. \&.

Dr. Bolben ring, Beh. 3uftizrat, Straßburg (Elfaß) Boetheitr. 11.

Dr. Bolbfelb, Sul., Redtianmalt, ডamburg, ABC=Strafe 6.

Bolbmann, Eb., Juftizrat, Berlin W., Botsbameritr. 22a.

Bolbmann, \&., Suftijrat, Miülbaujen i. E.

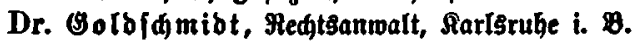

Dr. Bolbfdmibt, Brivatbojent, Berlin W., Lanografenitr. 9.

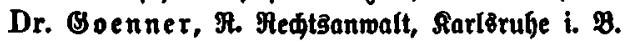

(S) onfdoromsi, Redtsanwalt, Stallupönen.

Dr. Boepel, 21mt Sgeridtstat, IItenburg S.:II., Langengaffe 10.

Dr. von Borbon, 3uftizrat, Berlin W. 8, DRobrenitr. 19.

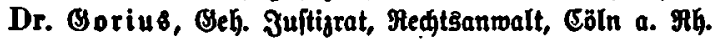

Goerres, Carl, Reditsanwalt, Berlin W. 8, CGarlottenftr. 59.

Go of mann, Georg, Fedtsanmalt, Notar, Jujtijrat, Bertin W., Maueritr. 66/67.

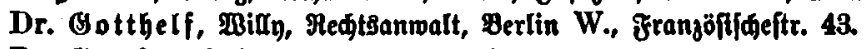

Dr. Gottfめalt, Ant'sgeridtstat, Solingen.

(Sottiめalt, Reopolb, Redtsanwalt, Berlin W. 8, Reipjigerftr. 112,

Dr. Bottfdalt, 5., Redtsanwalt, Seipzig, Thomasting 8.

Dr. Bottldalf, infreb, Reditsanwalt, Berlin W. Beisbergftr. 24.

Boeb, Carl, Br. Imtsridter, Rartsrube i. P., Malbftr. 66, III.

Boe \&mann, Medjtsanwalt, Majtatt.

Dr. Erabenwib, Brofefior, Straburg i. E.

Dr. von Orafen, Meditsanmalt, Suftizrat, Cöln a. Mh.

Dr. Oraff, Medtsanwalt, Jiteiburg i. B., Saiferitr. 152.

Grafer, Meditsanwalt, Malbghut.

Dr. Oraßboff, Medtsanwalt, Berlin W., gülowitr. 21.

(irau, 3uftizrat, Berlin N.W. 23, Flensburgerftr. 3.

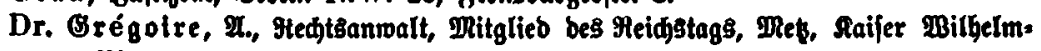

Ming.

(Sreiff, Oeh. Dbers:uftizrat, Berlin W., Rettelbedftr. 10.

Dr. Grelling, Ernit, Medjtsanwalt, Juitizrat, Berlin C., Spanbaueritr. 48.

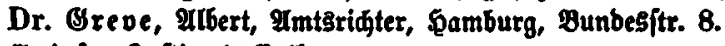

Oriefe, Juftigrat, Colberg.

Griefer, (E., AmtEgeridtsrat, Berlin, Ymtsgeridt Ditte.

Grobe, 5., Mectsanwalt, Motar, 3uiftizrat, Calbe a. Saale.

Srobe, Ilmtbrifter, Ibeisheim i Baben.

Groß, Friebrid, Redtsanwalt, MRergentbeim.

Srof, Fectsanwalt, Pforgheim,

Brolfe, Meferenbar, Maumburg a. S., \$ilh. Magneritr. 28.

\&roth, Ranbgeridttrat, \&anbsberg a. Marthe, \$eळoweritt. $30 \mathrm{~h}$. 


\section{XXVI}

Dr. Orote, 5., Midtcr, Bremen, Mathilbenitr. 27.

Dr. Ot rueber, Grw., Profeffor, Drũnden, Bifelaftr. 5.

Orumbaher, Dtto, Rectisanwalt, Railgrube i. $\$$.

(S) rünbaum, Redttsanmalt, Berlin C., Epanbauerbrüde 9.

Dr. (Srünbler, Carl, Meçtsanmalt, Jujtizrat, Berlin W., friebridjitr. 59/60.

Grūnebaum, Dberlanbesgeridterat, ఏamm.

Brîn[由ilb, Medtsanwalt, Berlin W., Seipjigerftr. 98.

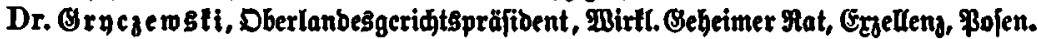

Dr. \&ugelmaiex, Bürgermeifter, \&oerrad.

Dr. Suggenheim, Mechtsanwalt, Dffenbad a. $\mathbb{M}$.

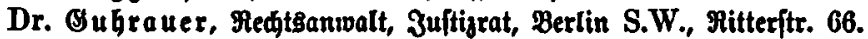

Dr. Güllanb, Lanoridter, Magbeburg, פeitenbjtr. 16 I.

(S) und, Franz, Rotar, Suftizrat, Elberfelo, Softamp 43.

Dr. (Surabje, Lanbridter, Cleve, sinbenallee 88.

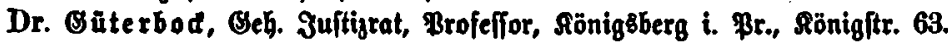

(8) utfelb, Medteanwalt, Berlin SW. 13, Gollmannitr. 21.

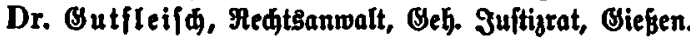

Guth, Rettanmalt, Rolar, Suftigrat, Seibe.

Dr. $\mathbb{G}$ utherg, Redtsanwalt, Beuthen, Babngofitr. 8.

Outmann, Dtto, Redt Sanwalt, Rartsrube i. B., Rarlftr. 13.

Dr. OUtmann, Sulius, Medtspraftifant, Sarlšrube i. B., Douglas|tr. 11.

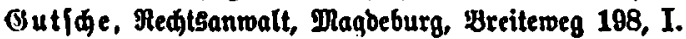

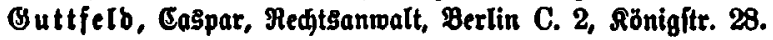

Guttmann, Julius, Medt5anwalt, Juftigrat, Bcrlin C., Mlofterftr. 83.

Suttmann, Ebuarb, Redtsanmalt, Magbeburg, Breitemeg 146.

(S uttmann, Mledtsanwalt, Rotar, Juftizrat, WBiesbaben, Mlorifftr. 4 .

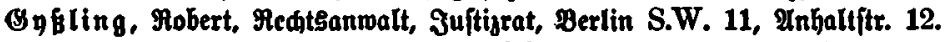

Saad, Dstar, Ecnatopräfibent, Berlin W., Joađimstbalerftr. 33/34.

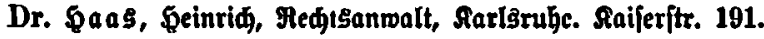

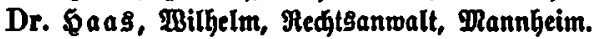

Dr. Бaas, \&., Medtsanwalt, Sarlstuhe i. $\mathscr{P}$.

Saas, Sanbgeridtstat, Saiferslautern.

Saas, Midjarb, Medtşpraftifant, Sarišrube i. $\mathscr{P}$.

Saber, Medtsantwalt, Suftizrat, Reipgig, Sdjwăgriđjenftr. 13.

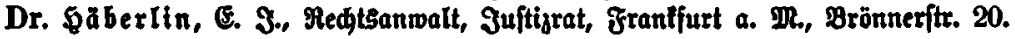

Dr. Saberling, \$8., Mediţanmalt, Daing, Raijeritr. 7.

Saberftroh, Ruguft, Ranogeridtsrat, Berlin N.W. 23, gadftr: 4.

Dr. \$ađenburg, TRax, Redtsanwalt, Mannbeim C. 2 R. 23.

Gä đermann, Medtantwalt, Suftijrat, Oreifsmalb.

\$abra, Irthur, Redtsanwalt, Berlin W. 8, Aanonierftr. 45.

Dr. \$afner, 21mtBridter, Maftatt, Raiferftr. 1.

Dr. \$after, E., Brofeffor, Rildbucrg b. Bürid.

Sagemann, Lanogeridt5rat, Mitglieb bes Meidjtags, Erfurt.

Sagemann, Pechtsanwalt, Notar, Tönning.

Sagen, Dlto, Rammergeridtstrat, Berlin W., Suitpolbitr. 11.

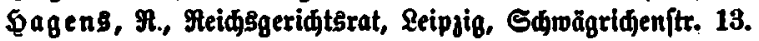

\$abn, 3ulius, Amtsgeridtsrat, Behlendorf, Aatharinenitr. 7.

\$abn, Frans, Medtsanwalt, Bexlin C., Rlofter[t. 77/78. 


\section{XXVII}

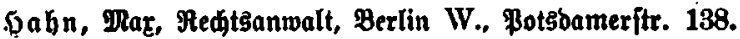

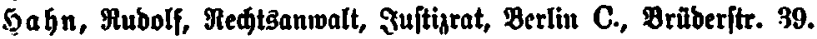

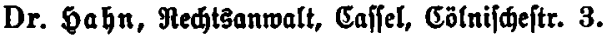

Da ib, Reditzanwalt, Meutlingen.

Dr. Saillant, Napoleon, Medttsanwalt, Berlin, Rammergeridjt.

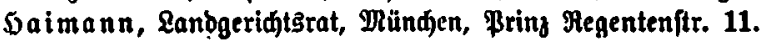

Dr. Salle, Medtbanwalt, Notar, Sujtizrat, Berlin W., Sronenftr. 56.

Sallensleben, Redisanwalt, Berlin C., Spanbaueritr. 63 a.

Dr. Salpert, Redtsanmalt, Berlin W., Botgbameritr. 4.

Dr. \$amburger, Baul, Bantier, Barlşruhe i. 9., Carlf́tr. 11.

Jamburger, Carl, Redtsanmalt, 3ujtigrat, Werlin W., BotBoamerftr. 118c.

Dr. Samburger, Carl, Bebeimer \$uftizrat, Mboolat, Motar, Franlfurt a. PR.

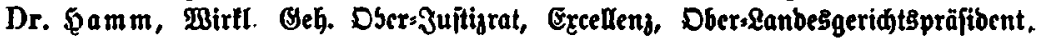

Bonn, Duantiusftr. 8.

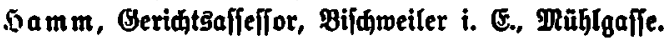

Sammer, Dberşujtijrat, Ebemnig (Eadjen).

Ja m meridj mibt, Rectsanwalt, Notar, Eottbus.

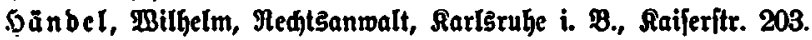

Sonot, Senatspräfibent, Eelle.

Sannemann, Rammergeridtstat, Perlin W., Dobjtr. 53.

ذannemann, Eriter Staatßanmalt, Ö̈rliks.

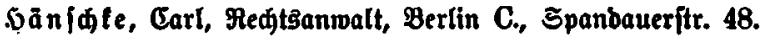

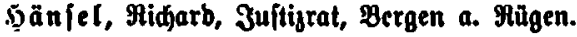

Dr. \$anfen, Qanbridter, Flenßburg.

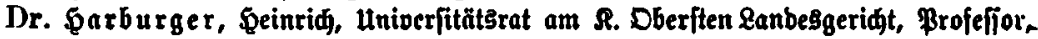
PRühłen, §arlftr. 21.

v. Garber, Ulteganber, Mectianmalt, Mannheim $\mathfrak{R}$. 2.1.

Sarbung, Redtsanwalt, Pannheim.

Dr. Sarmening, Ernft, Medtsanwalt, Sena.

Dr. Sarnier, Aug., Beh. Dber $3 u$ itizrat, Berlin W., Paffauer[tr. 4.

Dr. Sarnier, Co., Medtsanwalt, 3ujtizrat, Caffel, Cölnijळeftr. 3.

Jarrer, Mac, Mechtsanmalt, Raristuhe i. \$., Saijeritr. 89.

நarries, Redtsanwalt, Riel, Slinte 26.

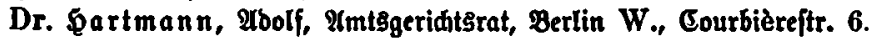

Dr. Eartmann, Lanogeriळtspräfibent, flenģburg.

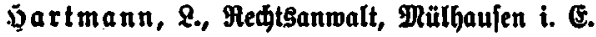

jo a tmann, Redtsanmalt, Charlottenburg, Sdilofitr. 8.

Jartwi

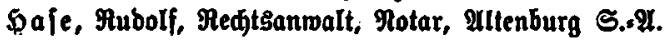

\$audecorne, Rammergeridtstat, Charlottenburg, Leibnifftr. 10.

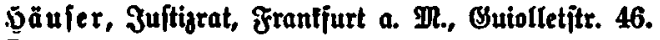

Dr. Бุaun

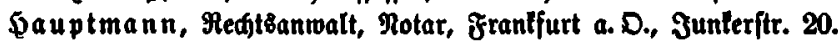

Dr. \$aufer, Meゆtsanwalt, Babens:Baben, Langeftr. 60.

Dr. Saymann, Frang, êfieffor, Frantfurt a. Di., Gervinugftr. 24.

Sayn, Sulius, Medtsanwalt, Berlin W., Begrenitr. 17.

Dr. Saทum, Redtsanwalt, Tübingen.

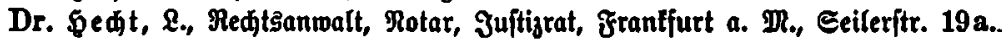




\section{XXVIII}

Dr. Sedt, Felix, Geh. Sofrat, Mannbeim.

bedt, C., Juftizrat, Medtsanroalt, Notar, Sanau a. PR.

Dr. Sedt, G.. Pedttanwwalt, MRannheim

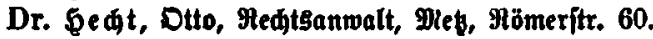

Dr. \$ed, Brofeffor, Tübingen, Retarbaloc 68.

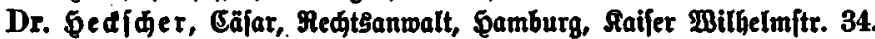

Sebbe, Medtsanwalt, Notar, Suftizrat, Dlarne i. Golftein.

Dr. Бegler, 2uguft, Profeffor ber Medtsmiffenjळaften, Tübingen.

Dr. Бeibemann, Medtşanwalt, Röslin.

Selbensleben, Amtsgeridtstat, Roftod i. IR.

Dr. Seil, Midarb, Međttsanwalt, PReb, Mömeritr. 60.

Setlberg, Medtsanwalt, 3uftigrat, Breslau, Ricolai:Stabtgraben 26.

Dr. \$eilborn, Brofeffor, Breslau V, Salvatorplat 8.

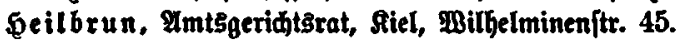

நeilfron, Brofefịor, Berlin W., \&afanenitr. 6.

Seiliger, Beheimrat, (Ëln a. Mh., \$obenitaufenring 47.

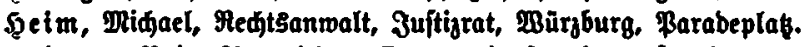

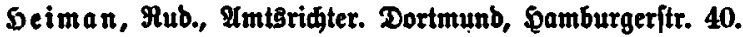

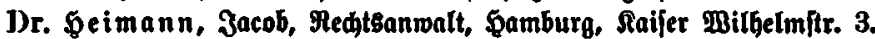

Dr. Бeimberqer, 3oleph, a. o. Profeffor ber Medite, Zonn a. Mh., Benusbergmeg 41.

Dr. \$einemann, Redtsanmalt, Berlin. Fronenitr. 8/9.

Dr. Seinemann II, Sans, 3ultizrat, ছannover, Beorgitr. 12.

Dr. Seinemann, 3ütizrat, Dlagbeburg.

Dr. Geinemann, Medtsanwalt, Motar, Gfen a. Mufr, Sagenftr. 50/52.

Seiniz, E., Juftizrat, Berlin W., פRohrenftr. 50.

Seinib, Grang, Medteanwalt, Berlin W., Bittoriaftr. 5.

Dr. Seint, Baul, Mbootat, Bürid, Sonnenquai 1.

Dr. Seinrici, Amtêridter, Dranienburg.

Dr. Seinsheimer, Carl, Profefjor an ber univerjität Seibelberg.

Eeinsheimer, Dtto, Redtsanmalt, Saristube, Raiferftr, 161.

\$eifer, \&rib, Meferenbar, frantfurt a. IR., Felbftr. 20.

Dr. Selfrib, Stabt.Synbilus, Greifsmalb, Moonftr. 6.

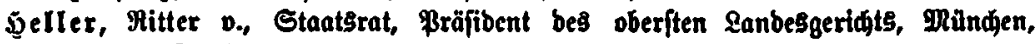
PRüluerítr. 31.

Dr. Selm, Medtsanmalt, \$eibelberg, \$auptitr. 222.

Dr. Gellmann, Friebr., Profeffor, Münden, Eabelsbergerftr. 1 a.

Dr. פellwig, Profeffor, Oeh. Jujtizrat, Berlin W. 15.

Sendel, \&ran, Medtsanwalt, Motar, Rienburg a. b. Wejer.

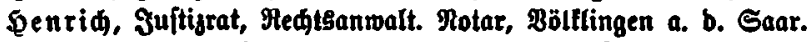

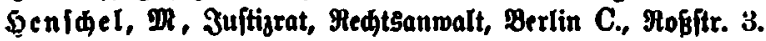

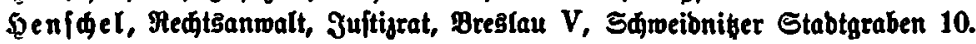

Eenjler, B., 2AmtEridter, Berghein a. Erft.

Serder, Moligang, Medtsanwalt, Berlin S.W., Eharlottenftr. 76.

Serbaน3, Rotar, Đilleşheim a. b. Gifel.

Germann, Malter, Medtsanwalt, Baben.Baben, Bernsbadjerftrafe 32.

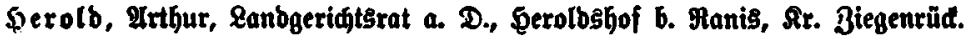

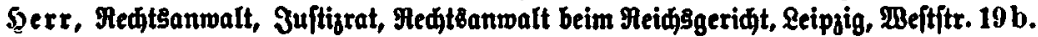

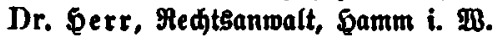


Serrenboerfer, Sultizral, Swinemūnbe.

Dr. Serrmann, Guitav, Medtక̧anwalt, Juftizrat, Berlin W., Mauerftr. 81.

Dr. Serrmann, Beorg, Itmtmann, Farlsruhe i. B., Friebridjplą 9.

Dr. Gerrmann, Ernit, Medtsanwalt, Baben=Baben, \&angeitr. 60.

Dr. Gerrmann, Ernit, Medtianwalt, Bonn a. Mh., Thomasiftr. 11.

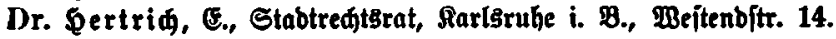

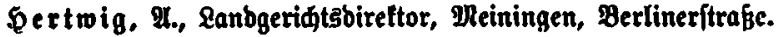

Dr. Ser

Dr. Ser, S., Senator, Gamburg, IRagbalenenftr. 3.

Dr. Sergfelb, Medtsanwalt, Effen a. b. Mlubr, Babnhofftr. 90/9:.

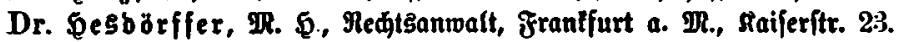

$\mathfrak{5} \subset \bar{B}$, Ilmtకriajter, Saatlouig.

Dr. Seß B, \&ubwig, Redjtsanwalt, Stutţart, Rirdjitr. 16.

Бeß B, OberlanbeSgeridtstat, Eelle, Rüneburgeritr. 10.

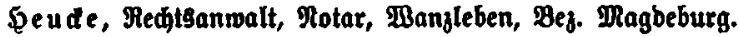

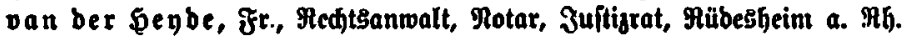

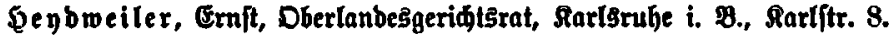

מener, 21., Motar, 3uftizrat, Berlin C., Friebrimftr. 114.

Dr. Genl, Snnbifug, Đamburg, UgneS[tr. 41.

Seymann, Juftizrat, Ultona a. E.

Seymann, Eduarb, Sammergeridtsteferenbar, Berlin W. 35, Rü(fowitr. 60.

Dr. jur. Geymann, $\mathcal{E}_{\text {., }}$ Profeffor ber Fledte, MRarburg a. b. \&.

joymann, 2ubolf, Fedtsanwalt, Berlin, Plumenitr. 39.

Dr. Seymann, Ebuarb, Rectstsanwalt, Dangig, Sundegafie 36.

Dr. Seymann, 5., Rechtsanwalt, Đamburg, Raijer Bilfelmftr. 23.

Dr. Geymann, Gang, Sdriftíteller, Berlin NW., In ben Belten 8.

Dr. Sid, Dberlanbesggeriatsrat, Rarlêrube (Baben).

Dr. Siebemann, Medtsanmalt, Cöln a. Mh., Elifenftr. 11.

Gilbebranb, 5. (5. F., Senator, Bremen.

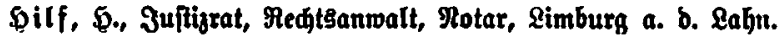

Dr. Ђilpert, 3uftjorat, tgl. Fovolat, Nürnberg, Đauptmartt 11.

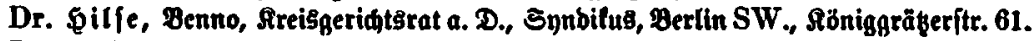

Dr. Đille, Carl, Synbitus, ßrofefior, Berlin SW., Söniggräķerftr. 61.

Dr. Winf, Dtto, Dberlanbesggeriatstat, Sartsruhe, Meftenbftr. 22.

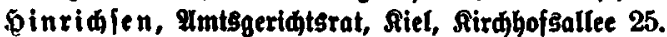

Dr. jur. Ginridfen, Robert, Rechtsanwalt, Notar, Súftrow i. 2 .

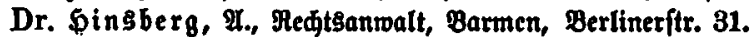

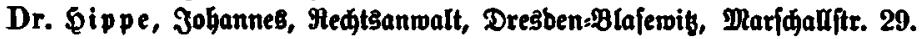

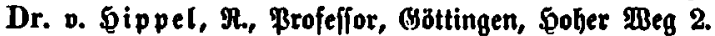

Dr. §irfd, Rarl, Geridtsafjeffor, Frantfurt a. $\mathfrak{R} .$, Meitenbitr. 52.

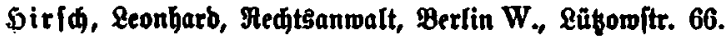

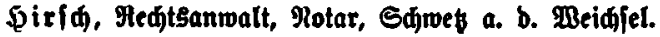

நirfafelb, Emft, Imtsgeriattstat, Eharlottenburg, Milmersborferftr. 80.

b̦iridffelbt, Medtţanmalt, Berlin C., Raifer Wilfelmftr. 18.

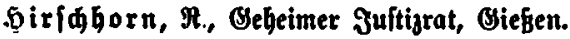

Dr. Sirfdlanb, Beorg, Effen a. o. Rubr, Betlwigeritr. 42.

Dr. Sirfdler, Fran, Medtsanwalt, Mannheim.

Sirfelorn, Felix, Redtsanwalt, Berlin W 8, Sanonierftr. 40. 
Dr. Soefer, Arno, Mectsanwalt, Altenburg, S.s\&.

Dr. Soffmann, $\mathbb{E}$, Wirtlider Beh. Dber = Megierungserat, Pinifterialbircttor, Berlin W., Courbièreftr. 10.

\$off mann, Redtsanmalt, Notar, Seybelrug, D./ Rr.

Dr. Soffmann, Sans, 3uftigeat, Berlin NW., Dorotgeenitr. 80.

Dr. \$offmann, Shax, Redtianwalt, Suftizrat, Rotar, Berlin W., unter ben Sinben 48/49.

Dr. Eolfmann, Redtsanwalt, \&oefnik.

Dr. Soffmann, Malter, Synoiłus, Megensburg.

\$offmann, Ernft, Redtsanwalt, Rotar, 3uftizrat, Bantow b. Berlin, Breiteitr. 30; \off ftaebt, 2 ., Yedtsanwalt, Berlin SW., Binumerftr. 19.

\$ofma del, Zriebrid, Eanbgeridtకprä[tbent, Traunjtein.

Dr. \$ölber, Beh. \$ofrat, Brofeffor, Seipzig, Sdpägridenftr. 26.

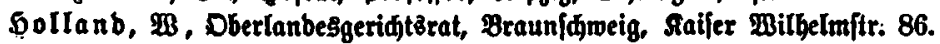

Dr. Sollaenber, Earl, Fectsanwalt, Berlin C. 25 , Mfezanberitr. 63.

J)r. Sollaenber, MIbert, Fedttanmalt, Samburg, Colonnaben 41.

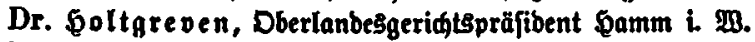

Dr. Solb, \&eo, Beh. Regierungsrat, vortragenber Rat im Mtinifterium für \&anbs wirtjळaft, Domänen unઠ Soriten, Ebarlottenburg, Berlinerjtr. 58, III.

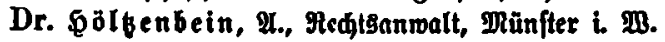

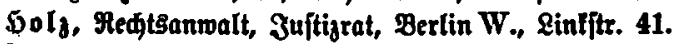

Dr. \$ols, Jerbinanb, Nedtsantwalt, Dannbeim.

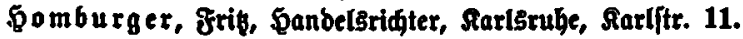

Sonig, Redtganwalt unb Motar, Bnejen.

Dr. \$̧onlg, \&., Medtsanwalt, Nürnberg.

Dr. Doeniger, $\mathfrak{G}$, Freiburg i. $\mathscr{8} .$, Bafiusftr. 54.

Dr. Soeniger, Lanogeriøtstat, Sarlşruhe i. $\mathfrak{B}$.

Dr. \$oepfner, 价efior, Eöttingen, Milgelm \$Beberitr. 24.

\$oeppe, C., Redtsanwalt, Meb, פadt Ttrafe.

Dr. Gorn, Fedtsanwalt, Berlin W 35, Görneritr. 4.

Đoerner, Fedtsanmalt, Pretten i. $\mathscr{P}$.

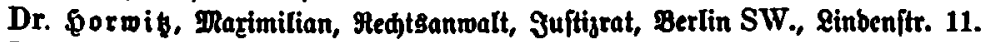

Dr. Sorwib̧, D, Reditsanwalt, Gamburg, Mofenbof.

Dr. Sorf́tmann, Bürgermcifter, Farlşruhe i. $\mathfrak{B}$.

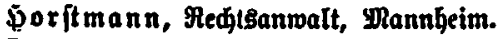

Dr. Soewig, 3., Redt5anwalt, BabensBaben.

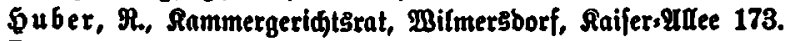

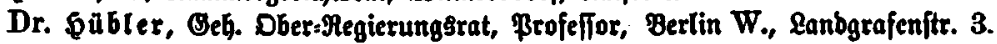

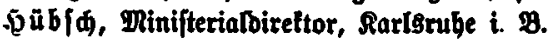

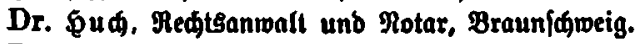

Dr. Gumbert, Juftizrat, Dlagbeburg.

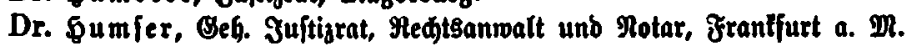

\$ünborf, Sultigrat, Đalle a. S.

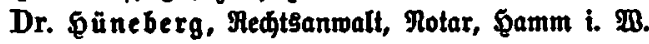

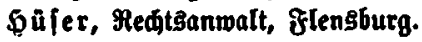

3acob, Emil, Beh. Rommergienrat, Berlin, \$olzmarttitr. 65.

3acob, Ilool, Notar, Soerrad.

Dr. 3acobi, Brofeffor, MRünfter i. 2 . 
Dr. Jacobi, Đerbert, Redtsanwalt, Münden, Färbergraben 7.

Jacobi, Redtetanwalt, Franlfurt a. $D$.

Jacobfofn, Mrac, 3uitizeat, Berlin W.9, Sintitr. 1.

Dr. Jacobfołn, Julian, Medtsanwalt, Berlin C. 2, Burifitr. 29/30.

Dr. Jacobjogn, 5., Redtęanmalt, Reipsig, Brü̆l 2.

3acobjohn, Mechtsanwalt, 3ujtizeat, Pofen.

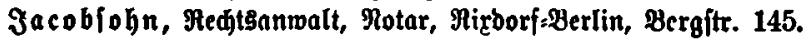

Jacoby, Ernit, Referendar, Berlin W., Bayreutheritr. 41.

3acufiel, Rurt, Medtsanualt, Berlin C., Raijer \$ilhelmftr. 17.

Dr. Jaffa, Sally, Redttanmalt, \$erlin C., Meue Friebridfitr. 21.

Dr. Jaeger, Dtto, Rotar, इodffelben.

Dr. Zaeger, Ernft, Profeffor, Leipjig, Plagmib̨, Forftttr. 2.

Dr. $3 a \mathfrak{h n}$, Mechtsanwalt, Prenglau.

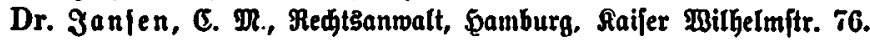

Sanjer, Subwig, Sartsrube i. 8 ., Eijenlohritr. 10.

3aredi, Medstsanmalt, Bofen, Reue Strafe 1.

Jasperien, Sanbelsridter, Fiel, gismard Allee 30.

Dr. 3elln id, Beb. \$ofrat, Seibelberg.

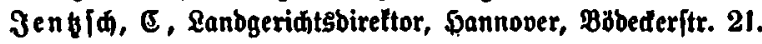

Dr. Befelfohn, Max, Redtsanwalt, Mannheim.

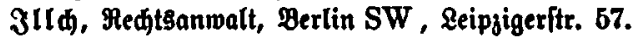

Imbers, I, Frang, Redtsanwalt, Berlin W., Botsbamerftr. 136.

Dr. 3mhoff, Eugen, Almtmann, Starlşruhe i. B., Reopolbitt. 7 b.

Dr. Smmerwahr, B., Bantbirettor, Berlin W., Meinedeftr. 25.

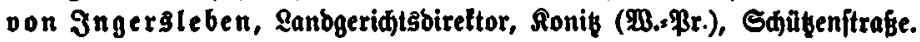

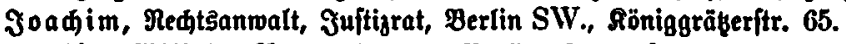

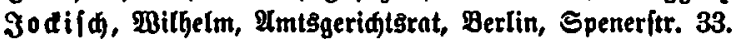

3obannien, Redtzanmalt, Notar, Ricl.

Dr. Jonas, Julius, Recttsanwalt, Altona, Fildutartt 27.

3ona S, \$aul, Medtsanmalt, 3uftizrat, Berlin W., Taubenftr. 16/18.

Dr. Jorban, Medtsanwalt, Mnanheim.

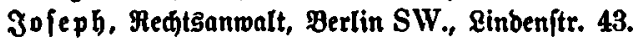

Dr. 3ofeph, Redttzanwalt, Frantfurt a. M., Bethmannitr. 46.

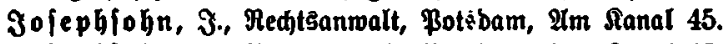

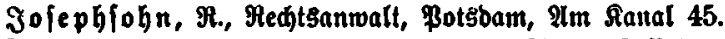

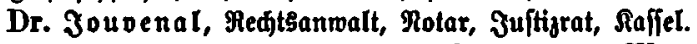

3rmler, Midjarb, Redtsanmalt, Suftizrat, Berlin W., Franzöftfdjeftr. 24.

Dr. Эlaac, Daartin, Medtaanwalt, Ycrlin, Jäger[tr. 63.

3laaciohn, Redtsanwalt, Berlin C., Raifer Milhelmitr. 13.

Dr. S

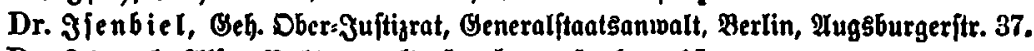

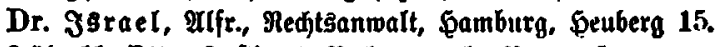

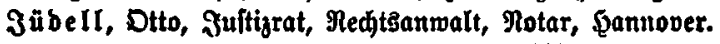

Juliusberg, Eanbgeridgtsbireftor, Berlin W., \$ragerftr. 29, II.

Dr. 3ung, Erid, Brofeifor, Greifäwald, Im Graben 3.

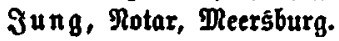

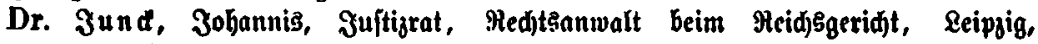
Zerdinand Mgobeitr. 38. 


\section{XXXII}

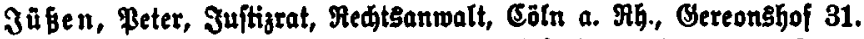

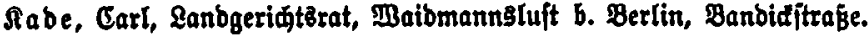

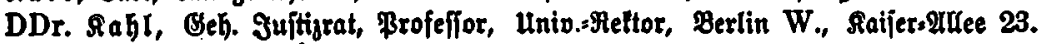

Dr. Aablenberg, 5., Bremen, Langeitr. 123/130.

Dr. Raehne, Meditsanwalt, Motar, Jujtizrat, Salle a. S., Broje Steinjtr. 14.

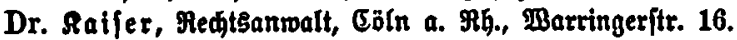

Dr. Raifer, Redtsanwalt, Reipjig, Bottjigebitr. 17.

Dr. Raifer, Medtsanwalt, Dres, Prieiterftr. 25.

Salif由er, \&anogeridtsrat, Berlin W 15, uhlanbitr. 169/170.

RaliBti, Mac, Redtsanwalt, Brestau, Sdmeibniłeritr. 43.

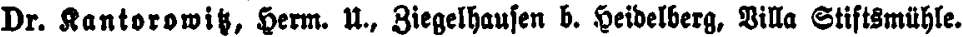

Rapferer, Medtsanioalt, Göln a. Mh.

Dr. Rar fiten, \&anbgeridtsrat, Berlin W 30, Moßjtr. 62.

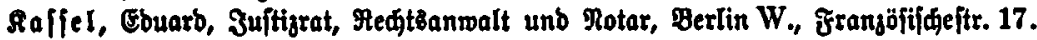

Dr. Ray, Ebrwin, Meditşanwalt, 3uitizrat, Berlin W. 64, Behreniftr. 17.

Rał, S., Medtianmalt, Berlin C., Spanbaueritr. 10.

Dr. Ra甘, Mobert, Me htsanwalt, Drannbeim.

Dr. Sauffmann, Carl, Redtsanwalt, Rannbeim.

Rauffmann, Ärthur, Referendar, Ehrenbreititein.

Dr. Raufmann, 27., ReGtşanwalt, Đeibetberg, Bienenitr. 1.

Dr. Raufmann, \$ugo, Mechtsanwalt, Rrefelo, Morbwall 75.

Faufmann, Emil, Fectsanwalt, Pragbeburg, Gumbolbitr. 2.

Dr. Raufmann, Frik, Fedjtsanwalt, Mannbeim C. 2.2.

Dr. Raufmann, Guftav, RechtEanwalt, Mlannheim C. 2. 2.

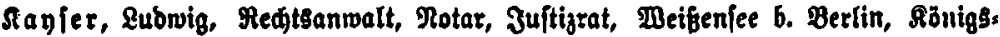
中аuffee 52.

Rayjex, Rotax, Babern (Eljaß $)$

Red, S., Mechtsanmalt, Rotar, Segeberg.

ReIler, Dberlanbesgeridtstat, \$amm.

Dr. Reller, Brivatbozent, Bonn a. Mh., Raijeritr. 95.

Dr. Reller, R., Medtsanwalt, Pannheim.

Dr. ReIler, Y., 3uftizrat, Notar, Straßburg, Bioniergaffe 7.

Rempf, Redtsanwalt, Suftizrat, Bertin W., unter ben Sinben 30.

Dr. Remmeter, Medtsanwalt, Rempten.

Rempner, AmtBggeridtErat, Branbenburg a. $\mathfrak{b}$.

Renres, Međtsanwalt, Potsbam, Im Ranal 57.

Rerfting, Ranbgeridtebirettor, Riel, Bartelsallee 9.

Dr. Reffel, Rechtsanmalt, 3uftizrat, \&udau i. \&.

Dr. Renßzer, Alífior, Bantoro b. Bertin, Breitejtr. 41.

Dr. Riefe, willyelm, Medtsanwalt, Stuttgart, \$eraitr. 3.

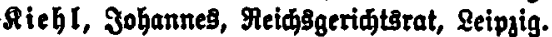

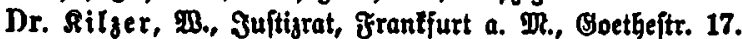

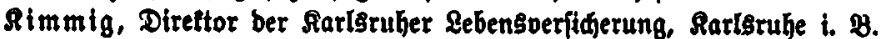

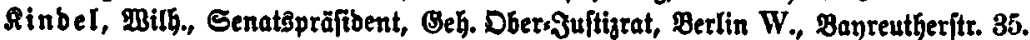

Dr. Ripp, Brofefior, Berlin W., DReinedeitr. 20.

Dr. Rirdenbauer, R., Reditsanwalt, Sarlsrube i. $\mathscr{B}$.

Dr. v. Rirfienbeim, Profefior, Geibelberg.

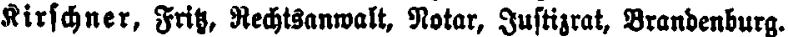

Dr. Rifd, univerfitäts:Profefior, Straßburg i. E., Sdjwargmalbjitr. 6. 


\section{XXXIII}

Rik, Ranbgerid)tspräjibent, Srefelb.

Rlaholt, Medtanunalt, Notar, Belienliudien.

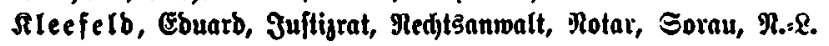

Rleefelb, Medtsanmalt, Borms a. Mh.

Dr. Rlein, Redtsanwalt, Colmar i. E.

Dr. Rleinfeller, Profeffor, Siel, Riemannsweg 101.

Rle in a th, D., gtedtsanwalt, Бannover, Bahnhofitr. 12.

Dr. Rlenter, Redtsanwalt, Fonn, Rönigitr. 41.

Rle y, Notariatsinjpeftor, Rartsrule i. $\mathfrak{B}$.

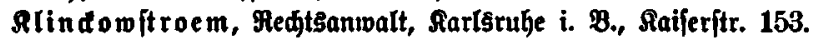

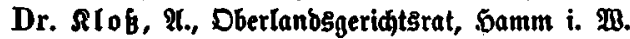

Dr. jur. et phil. Rloeppel, E., Elberfelb, 8Bismarditt. 17.

Rlob, Ernft, Medtsammalt, Dresben, Maridaulftr. 18.

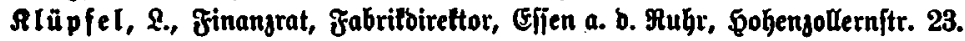

Rnebel, Paul, Rectisanmalt unb Motar, Behlenborf, Sauptftr. 1.

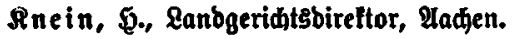

Dr. Iniep, Oeh. 3ujtizrat, Brofeffor, Jena.

Dr. Anittel, Midjarb, Rarlşrube i. B.

Dr. Anopf, Albert, Medtåanmalt, Berlin SW., Reipjigeritr. 59.

Rnäpfler, Qeh. 3uftizrat, IRarienwerber i. Meftpr.

Soebel, Fedtsanmalt, Colmar i. E.

Rod, Conrab, Itmtšridter, Molbenberg.

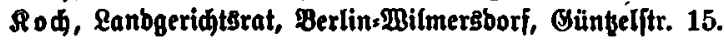

Rod, Max, Redit3anwalt, Berlin W., Rotsbamerftr. 13.

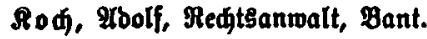

Rodmann, Beorg, Redtsaumalt, Bleimiţ, Micolaiftr. 13.

Dr. Roderols, Motar, Suftizrat, Roblend, Sdilofiftr. 6.

Roffe a, Jujtigrat, Bertin W., Reujtäbtijde Rirdftr. 1.

Dr. Roffta, B., Sammergeridts: Senatspräfibent, Berlin NW. 23, Siegmunbshof 16.

Dr. Roebler, 2 ., Nedytsanmalt, Mannheim.

RöGler, Imtsididter, Flatow i. Weftpr.

Dr. Robler, Pluguft, a. o. Univ.: Projeffor, MRinden, Therefienftr. 2.

Dr. Rohlrauโd, E., a. D. Profeffor, Söniggberg i. \$r., \&uijen=QIIee 36.

Rohn, Fr., Fedtsanwalt, Dortmunb.

Dr. jur. et phil. Röhne, Carl, Privatbojent an ber tedijniden \$odjijule, Berlin W., Seitbftr. 12.

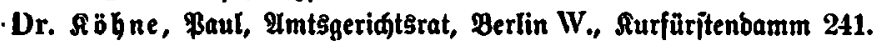

Stoelle, Itmtsgeridtstat, Clausthal.

Roenig, Jriebrid, Redtsanwalt, Nannheim.

Dr. Roenigsberg, Reopolb, Reфtsanwalt, Бamburg, Bänjemartt 21/23.

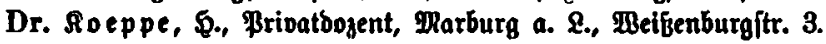

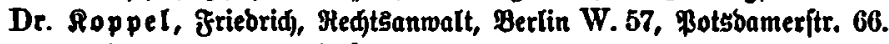

Röppl, Medtsanwalt, 3ujtizrat, Pelgarb (Pommeril).

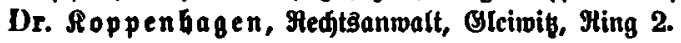

Rortum, Sarl, Beh). Jujtizrat, Raumburg a. b. Saale.

Röfter, Eanbgeriøtsbireftor, Berlin W., Bayreutherftr. 10.

Dr. Soettgen, Seh. Sultigrat u. vortragenber Rat im Suftizminifterium, Berlin W., Surfïritendamm 117/118. 


\section{XXXIV}

Sraft, Medtsanmalt, Berlin W., Potgdamerftr. $122 \mathrm{c}$.

S rah, Sanbridter, Riel, Dammitr. 21.

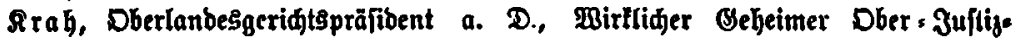
rat in Riel.

S rapp, Lorenz, fgl. Dberlanbesgeridtstrat, Iugsburg, Stettenftr. 28.

Dr. Rratt, Medtsanwalt, Pforbheim.

Dr. Sraemer, Milbelm, Medtsanwalt, Berlin W. 35, ßotsbamerftr. 55.

Dr. Rrater, Redtsanwalt, Rarts̊ruhe i. $\mathscr{8}$.

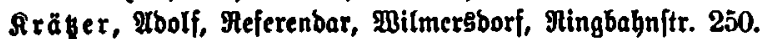

Iraus, Dberlanbesgeridttrat, Riel.

Dr. Araufe, Beh. Suftizrat, Berlin W., Behrenftr. 24.

Dr. von Rraewel, Lanbridter, Duisburg, Em/djeritr. 12.

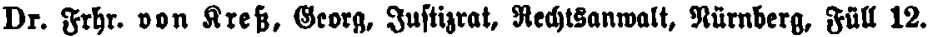

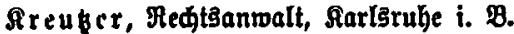

Dr. Rriegsmann, $R$. Serm., Privatbogent, Ricl, Rnooperitr. 47.

Dr. Rrobne, Berlin W., Rürnbergerítr. 25/26.

Dr. Ironeder, Ernit, Sel. Jultigrat, Berfin, Charlottenburg, Shlüteritr. 34.

Dr. Aroner, Seinrid, Fedtsanmalt, Berlin W., Leipsigeritr. 129.

Dr. Aronfelb, Fedt tsanwalt, Jujtijrat, Berlin W., Reipjigerjtr. 100.

Dr. Rrü đmann, Paul, Profeffor, Mlünjter i. $\mathfrak{B}$., Burdharbftr. 14.

Arug, Medtsanvalt, Sranffurt a. MR., Savignyitr. 6.

Dr. §rüger, E., Dojent, Ealw.

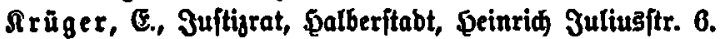

Rrüll, Georg, Medtsanwalt, Glberfelb.

Rrumbiegel, Fotar, Elberfelb.

Rủbler, Geh. Dber=\{uftizrat unb vortragenber Mat im \}uftizminifterium, Berlin W., fturfürften[tr. 56.

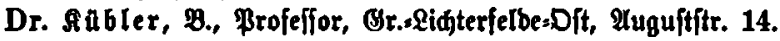

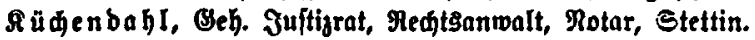

Suful, Mectisanwalt, Blantentefe.

Rü hn, Ernit, Redtకanwalt, Motar, Meerane i. S.

Fulemann, \$B., Eanbgeridtsrat, Bremen, Faifer Friebridftr. 15.

Dr. Sulenla mpff, Зul., Redtsanwalt, Bremen, Domaheibe 3.

Sulenfampff, \$erm., RedtEanwalt, Bremen, Domsjeibe 3.

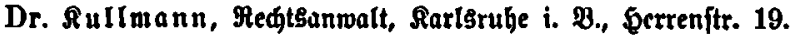

Summerfelo, Amtsridter, §annoder.

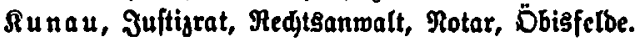

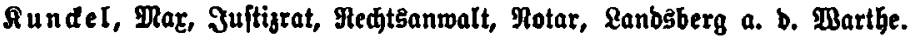

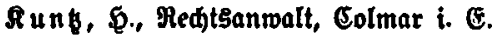

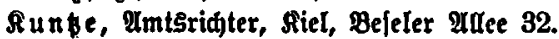

Rũn bel, Dbersanbesggeridtsrat, Raumburg a. S., Breithauptftr. 3.

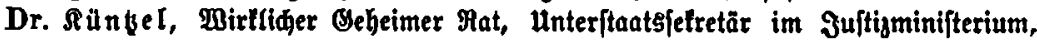
Ezzellen, Berlin W., Genthinerftr. 39.

Rurlbaum, Feditsanmalt, Reipzig, Scbaftian Badjtr. 7.

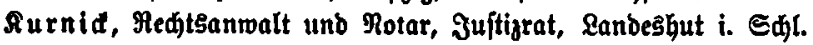

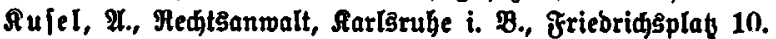

$\Re$ üfter, Meferenbar, Siel.

Eabewig, Frik, Redtsanwalt, Berlin W. 9, Kotsbameritr. 132. 


\section{XXXV}

Sagro, Meditsanwalt, Berlin W. 66, Maueritr. 78/79.

Qähr, 2Tuntsgeriđtsrat, Belgarb (Ponımern).

\&amp, Theodor, Medjţanwalt. Rotar, Elmshorn i. Solftein.

Dr. \&ampredt, Eanbridter, ఏamburg, Sophienterraffe 3.

Sandau, Koolf, Mecttsanwalt, Berlin C., Furftr. 20/21.

Dr. Lanbau, Feliz, Medtకanwalt, Juftizrat, Berlin W., Jägerftr. 58.

Ranbé, Imtsridter, Berlin W. 15, Olivacrplaß 8 .

Dr. Ranbsberg, Imtšridter, Reumuniter.

Eanbsberg, Hedtsanwalt, Juítijrat, Pojen, Zriebridjtr. 5.

Eanbsberger, Felic, Nedtsanwalt, Dets (Edlefien).

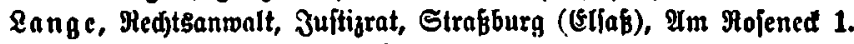

Dr. \&angen, Profefior, Müniter i. $\mathfrak{B}$.

Dr. \&angenbad, Beriditsaffeffor, TRorms, Boetheftr. 15.

Saster, 3ujtizrat, Lanosberg a. B.

Laué, \$alter, Redtiłanwalt, Notar, Juftizrat, Berlin SW. 68, Rodfftr. 9.

Dr. \&ar, Ifffeffor, Bielefelb.

Lazarus, Di., Medtzanwalt, Juftizrat, Berlin SW., Rindenjtr. 112.

Dr. Ecanber, Fedtsanwalt, Berlin SW, Sulmbadjeritr. 7.

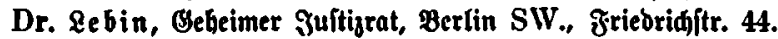

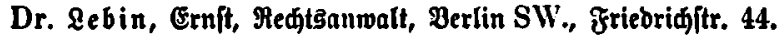

Qeers, Mechtsanwalt, Berlin W., Leipzigerftr. 08.

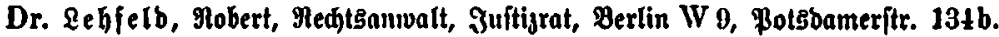

Dr. Sehmann, Irfeffor, Berlin W., Siurfüritenbamm 26.

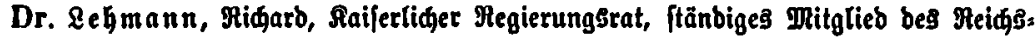
verjitjerungsamts, Brunewalb, Snausftr. 10.

Dr. \&ehmann, Sarl, Profeffor, Moftod i. Dr., Paulftr. 52.

Dr. \&ef) mann, פeinrid), ßrivatbojent, Bonn a. Mh., Irgelanberftr. 124.

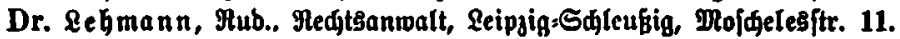

Dr. \&e hmann, RechtsanmaIt, BotEbam, Branbenburgeritr. 25.

Dr. Lebmann, Fedttsanwalt, Stettin, Slofengarten 1.

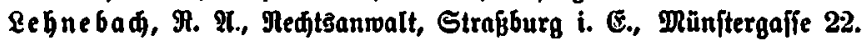

Dr. Rehnien, 3ohannes, Dojent ber Medte, Seibelberg, Raijeritr. 11.

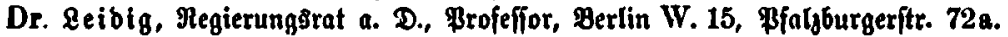

Dr. Leipaiger I, Beorg, Nedtsanwalt, Breslau, Ning 25.

Eeifer, Julius, Medtţanwalt, Meţ, Raifer Bilhelm Ming.

Dr. Remberg, Arthur, Red)tsanwalt, Bres(au, Sdjweibnifjer Stabtgraben 10.

Dr. \&enel, Dtto, Brofeffor, Freiburg i. :., Bafiusftr. 47.

Dr. Rennis, Redtsammalt, Straß̧6urg i. Elfaß̧, Broplieplat 20.

Qenzbers, Beorg, 3uftizrat, Sannover, Sittilleriejtr. 29.

Dr. \&eonljarb, \&ran, univerfitäts:\$rofeffor, Marburg.

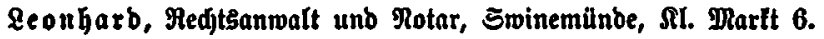

Dr. Seonharb, Mubolf, Seh. Juftijrat, Profeffor, Sreslau, Rleinburg, Sinbenallee 6.

Dr. Lefer, Walter, IImtstidater, DRannheim, M. 7. 1 a.

Seudert, Sanogeridtstat, Straßburg i. E., Bwingeritr. 3.

\&evi, Redtşanwalt, Sujtigrat, Bonn, Medenleimerftr. 64.

Eevi, R., Medtsanmalt, Stuttgart.

Dr. Revi, Sigmuno, Redtsanwalt, Rain, Fładjsmarlt 3.

Dr. Revin, Mobert, Mlectianwalt, Berlin NW., Moonftr. 10. 


\section{XXXVI}

Dr. Sevis, Dtto, Dberamtsriditer, Pforgheim, \&inbenftr. 17.

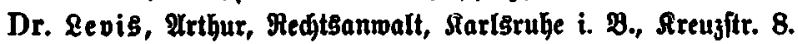

Qevot, Dr., Rebtsanualt, Juftizrat, EöIn a. Mh., Elifenitr. 23.

Dr. \&evy, 3. ม., İbotat, Imfterbam, Mecrengradt 210.

Levy, Seoport, Imtsididter, Senftenberg.

Dr. 3. Le vy, Mebalteur ber Doffifden Beitung, Berlin C., Breiteftr. 8.

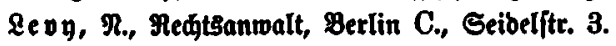

\&evy, Sultug, Medténanalt, Berlin C, Stralaueritr. 13/14.

Dr. Revy, Meditsanwalt, Eaffel.

\&ewalb, C., Beheimer Jujtizrat, Leipgig, Simjonjtr. 2.

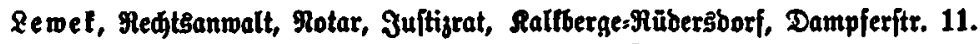

Sewin, G., Antsgeridtbrat, Magbeburg, Dranienítr. 11.

semin II, Submig, Medtsanwalt, Berlin W, PotBbamerftr. 23.

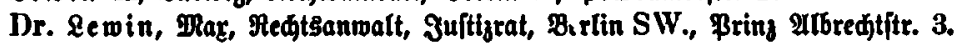

Dr. Lewin, Medstsanwalt, Stettin, Breiteftr. 33.

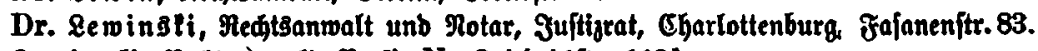

Bewingti, Redtsànmalt, Berlin N., Friebridftr. 112b.

Rewing $1 y$, Moolf, Redtsanwalt, Berlin SO 26, Dresbenerjtr. 135.

Rewinsty, Redtsanwalt unb Rotar, Danjig=Sangfuhr.

Dr. Leminfohn, 3ofef, Medtsanwalt, Berlin W., Jägerftr. 20.

Dr. \&eyfer, Эuftigrat, Medtsanwalt, Egarlottenburg, Berlineritr. 161.

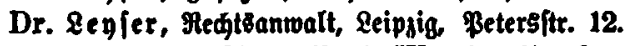

Siebenthal, Suftigrat, Berlin W, Benthinerftr. 22.

Dr. Riebinedt, Rarl, Redtsanwalt, Berlin N., 厄hauffeeftr. 131.

Dr. Rieble, Redtsanwalt, Rotar, Flengburg.

\&iebtte, Mehtsanwalt, Berlin W., Sutherftr. 48/40.

Dr. Etefmann, Mobert, Profeffor, Freiburg i. B., Goetbeitr. 33.

Etepmann, Profephor, Siel, Moltteftr. 42.

Dr. o. Sillenthal, Rarl, Brofeffor, Beheimer Sofrat, Geibelberg, Biegethaufer Sanbftr. 30.

\&ilienthal, Redtsanwalt, Juftizrat, Berlin C., Breiteitr. 11.

Dr. Lindelmann, Feđt

Linded, Mnton, Meḑtsanwalt, Mannbeim

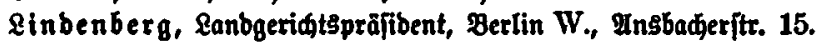

Dr. \&ippmann, Jul., Medtşanwalt, \amburg, Bleidenbrüde 10.

Dr. v. Sippmann, Carl, Sgl. bayer. Beh. Mat, Meidģgeridtstrat a. D., Steglił.

\&isco, 3uftigrat, Berlin W., Dlohrenftr. 13/14.

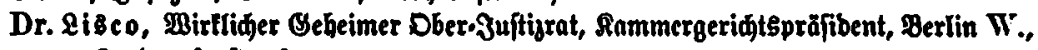
Lanografenftr. 9.

Dr. Siffauer, Rectsantwalt, F̧ürtenwalbe.

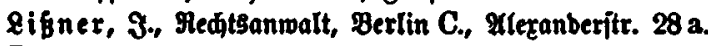

Dr. v. \&isjt, \&rans, Beh. 3uftijrat, \$rofeffor, Egarlottenburg, Sarbenbergftr. 19.

Dr. jur. \&itten, a. o. \$rofeffor a. b. Unio. Galle a. S., Burgftr. 43.

Dr. Soe b, Medteanmalt, Dannheim.

Dr. Sobe, Dberlanbeģgeridṫrat, Dreşben, Meiffigeritr. 9, II.

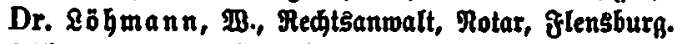

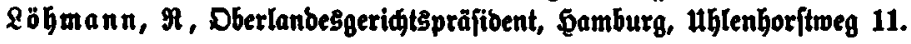




\section{XXXVII}

Dr. Soehr, Jojeph, Direttor ber Bayer. \$anbelşbanl, Igl. Oriedjilder Benerals tonful, MRünden, Qltabemieftr. 11.

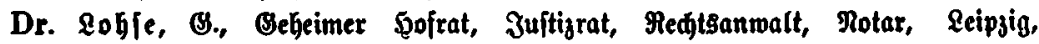
Ratgarinen|tr. 20.

Qoble, Medtsanwalt, Dlbenburg i. Or.

Somnif, Redtsanwalt, Berlin C 2, Reuc Bromenabe 2.

Dr. Rood, Itmtstidter, Berlin, Imtsgeridt Mitte.

Qorenz, Lanbridter; Bertin, Thomafiusftr. 20.

Dr. \&oren , Medtæanwalt, Sarlsrube i. B., Raiferftr. 89.

\&ufer, Dttomar, Nedtanmalt, frantfurt a. b. DDer.

Dr. Roffen, Oberlanbesgeridtstrat, Colmar i. E.

Dr. phil. Botmar, Brofeffor, Bern i. Sdrweij.

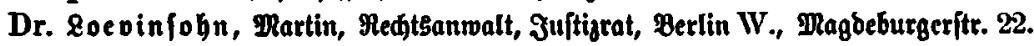

\& öwe, Ranbgeridttsrat, Perlin W., uhlanbitr. 30.

Eoewe, F., Medttsanwalt, Berlin NW., Friebridftr. 154.

Ioewe II, Ilphons, Redtsanwalt, Spanbau, Motsbameritr. 40.

Dr. Qoewenfelb, Redtsanwalt, 3uftizrat, Berlin NW., Mathenuweritr. 78.

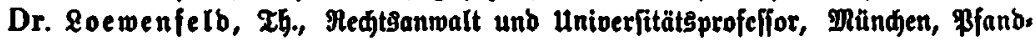
bausftr. 3, I.

Qoewenftein, Medtsanwalt, Paberborn i. 2 .

8swenthal, Dtto, Redtsanivalt unb Notar, IItona, Mlopitoditr. 25.

8owenthal, ff, Redtsanwalt, Notar, Sdmerin (Dedlenburg).

Eoewenthal, Tax, Medtsanwalt, Berlin W., Ranonieritr. 8.

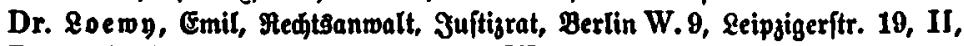

Dr. Rubsjinsli, Rectsanwalt, Berlin W. 21, Potsbamerftr. 23a.

Dr. \&ucas, Dhinifterialbireltor, \$irtl. Beł. Ober-Jujtizrat, Bertin W., Ilden= badiftr. 6.

Quderath, C., Rechtşanmalt, Rotax, Mettmann.

\&ubwis, Pring von Bayern, Rgl. \$oheit, פRünden.

Dr. Sanenburger, Redtsanwalt, Strafburg i. E.

Eunglmayr, Dberlanbesgeridtsrat, 3meibrüđen, Maxitr. 19.

\&urie, Juftizrat, Stettin, Friedrid Carlítr. 35.

\&uftig, Eugen, Reditsanwalt, Suftizrat, OSteimiz.

Dr. Qü gebrune, Walter, Referenbar, Böttingen.

\&ütgens, Meferenbar, \$amburg, Eppenborferbaum 8.

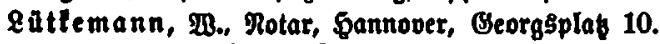

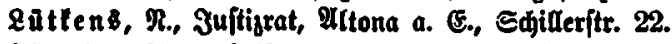

Eü

Dr. Mr a as, Georg, Bibliothetar am MetdSmilitärgeridt, Beblenborf, Berlinerftr. 8.

Dr. Pa as, Ranogeridtsrat, Rartsrube i. $\mathfrak{B}$.

Madol, Medtsanwalt, Rönigitein a. Taunus.

Magnuร, Sulius, Fechtzanwalt, Berlin NW., Dorotbeenjtr. 49.

Pabla, Frtebrid, 3útizrat, Motar, Dürtfeim.

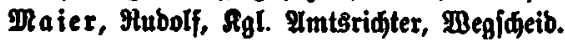

Mainharb, Ranbgeridtsిrat, Rarlsirube i. Baben.

Dr. Mall mann, \&anbgeridjtsbireltor, Cöln, Gokenftaufenxing 43.

Manalfe I, Medtianwalt, Grünberg. 


\section{XXXVIII}

Dr. phil. et jur. SRanegి, Profefior unb Generalfefretär bes beutidjen Bereins

für Berfidjerungs,\$Biffenjdaft, Berlin W., Bragerftr. 26.

Prantiewiz, Beorg, Medtęantwalt, 3ultigrat, Berlin W., Bebrenjtr. 22.

Dr. $\mathbb{R a n n}_{\mathfrak{n}}$, Midarb, Redtsanmalt, Frantentbal.

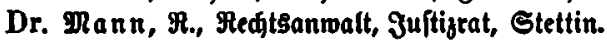

Ranien, 3ujtizrat, Dortmunb, Ruomigítr. 10.

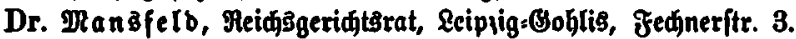

Marcus, 3., 2mtsgeridtstat, Berlin S. 59, \$ajenteibe 67.

Marcus, Buftav, 3ujtizrat, Buben.

Marcus, Redtsanwalt, Berlin W., Ebarlottenjtr. 48.

Marcule, Th., Medtsanwalt, Berlin SW., Friebridftr. 226/27.

Darggraf, Serrmann, Medttganmalt, Berlin W., \$otgbameritr. 46.

Dr. Mard, Mubolf, Redtsanwalt, Dannlyeim P. 7, Seibelbergeritr. 18.

Dlartwalb, Afezanber, Medtsanwalt, Juftigrat, Berlin W., Mobrenftr. 10.

Dr. Dartin, M., Senatspräfibent, Samburg 37, פBerberitr. 42.

Mtartin, Eugen, Suftizrat, Medttsanmalt, Rürnberg. Mbler|tr. 33.

Dr. v. DRartił; Dberverwaltungsgeridtstat, Profeffor, Berlin W., frajanenftr. 93. IRarun, Meditsprattifant, Sarlšruhe i. $\mathscr{P}$.

Marz, Ituguft, Medtsanwalt, Mannhcim.

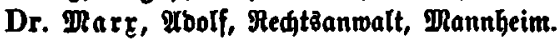

Dr. Parx, Medtsanwalt, Rarişruhe i. $\mathfrak{B}$, Raijeritr. 85.

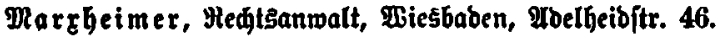

Trafdle, Meditsanwalt, Rotar, Roniß i. Meitpr., Denlmalsplak 7.

Matthaei, \&., Amtsgeridtstat, Sunnower, Sophienftr. 6.

Dr. Mattes, Medtsanwalt, Bemeinberat, Stultgart, Mathaus.

Dr. Matthias, Profeffor, Moftod i. M., Stefanjtr. $13 a$.

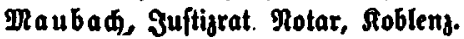

Maur, Juftizrat, Cobleng a. $\Re$.

Maurer, Carl, Eanbgeriditsat a. D., Baben:Baben, \$olgbofitr. 1.

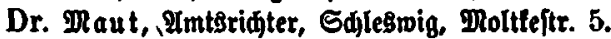

Dr. Ma ver, Carl, Red)tEanwalt, Jultizrat, Cöln a. MG., Beorgitr. 5 a.

Dr. Mayer, Morik, Medtsanwalt, Frantenthal (Pfald).

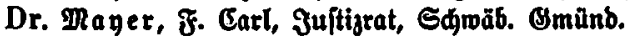

Dr. phil. et jur. Mayer, Max Ernft, Profefior ber Medte, Privatbogent, Strapis

burg i. E., Utniverfttätsittr. 38.

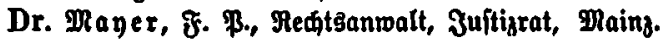

Maner, Bernharb, Rechteanwalt, Straßburg i. E.

Dr. Dayer, Guitav, Redttsanwalt, Dannheim.

Mayer, Ostax, Meditananalt, Mannheim 0. 4. 16.

Dr. $\mathfrak{Z}$ ayer, Otto, Profeffor, Reipsig, Aronpringltr. 15.

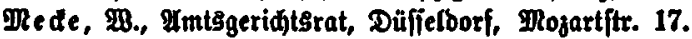

Mehnert, Carl, Medtsanwalt, Rltenburg, S.M.

Deibauer, Mectsanmalt unb Rotar, Flatow i. Weftpr.

Deier, \$., Beh. Juitizrat, Siel.

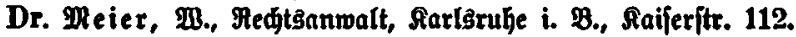

Dr. Meier, Redtsanwalt, Pforjheim.

Meinbarb, Medjtsanwalt, Berlin W., Reipjigerjtr. 98.

Meinginger, Medtianwalt, Landau. 


\section{XXXIX}

Meisner, CGriftian, Medtţannalt, Mürzburg.

Deifter, Redtsanwalt, 3uftizrat, Stettin, Bismarditr. 6.

Dr. Deifter, Beridtsaffeffor, Brudial.

Dr. PRenbel, Rectşanwalt, Dresben, Daridjallftr. 27, I.

Denzen, Medtsanwalt, Bonn a. $\mathfrak{N h}$.

Dr. Denzen, Beridtšrat, Frantfurt a. DR. = Ejđersheim, Billa (Shrifta.

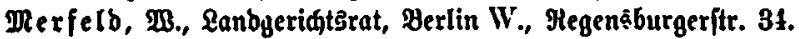

Derdle, Medtsanmalt, Frantenthal (PfaI $\left.I_{3}\right)$.

Merl, Malter, Meditspraftifant, Farlşruthe i. B., Belfortittr. 10.

Dr. Perfel, M., Brofeffor, Freiburg i. B., Baftusitr. 49.

Deridberger. Staatsanmaltidaftsrat, Bilmersborf, Xanteneritr. 1.

Merzbader, 3uftizrat, Rürnberg.

Dr. PRefdelfoljn, Su[tigrat, Berlin W., \$ohenjollernitr. 24.

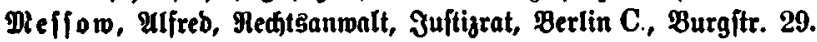

Me

Dr. פre e l, Brivatgelehrter, Stettin, Linbenfir. 21, p. l.

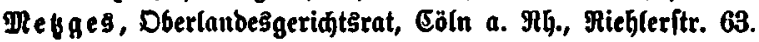

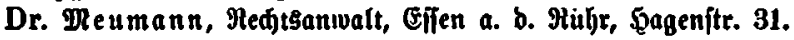

Dr. DReurer, Brofeffor, Würburg.

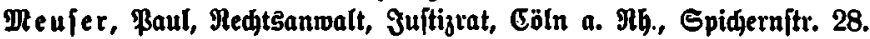

Dr. med. et jur. Dle yer, Th., Frivatoogent, Jena, Eerberitr. 3.

Dever, Amtsgeriøtgrat, Charlottenburg, Bleibtreuftr. 44.

Dr. Dever, frif, 3uftizrat, frantjurt a. $\mathbb{R}$, Meue Zeil 63.

QRever, Dtto, Eberlanbesgeridtstat, Celle, Şannoverideftr. 3, I.

Dr. DReyer, Dsfar, Redtsanwalt, Berlin SW., Delle-2(rianceftr. 10.

Meyer, \$aul, Rechtsanwalt, 3uftizrat, Berlin SW., Friebriditt. 239.

Dr. DRe yer, Friebria, Arthur, Redtsanwalt, Berlin SW., Bellesallianceplaß 3.

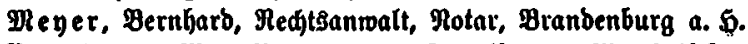

Dr. MRe yer, Mat, Redtsanwalt, Zrantfutt a. MR., Stiftitr. 22, III.

Deyer, Eugen, Hedtsanwalt, Freifurg i. $\mathfrak{B}$.

Dr. Me yer III, Subwig, RedtEanmalt, Eannover, Sdjillerftr. 32.

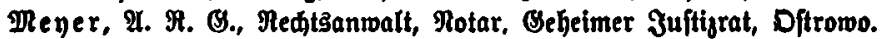

Me yer, Eomin, MedtEanmalt, Rotar, Jujtizrat, Tilfit, Fafernenitr. 24.

Dr. Reyer von Sdauenfee, Pl., Dbergeridtsoizepräfibent, \&uzern.

Meyerhoff, Beorg, Rewtsanmalt, Ricolasfee, Ribellen/trafe.

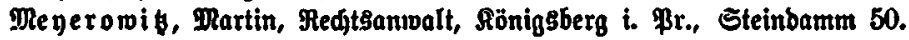

Meyerftein, Ebuarb, Meditsanwalt, Syndifus ber Sanbelstammer, Berlin NW. 7 , Dorotheenitr. $7 / 8$.

Dr. Pley ģner, Erid, Jujtijrat, Berlin W., Gronenftr. 73/74.

Ridaelis, Ridjarb, Dberlanbesgeridtstat, Colmar i. E.

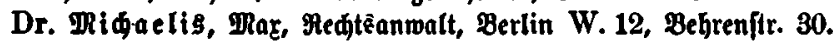

Dr. Miđa els̄, Malter, Medtsanmalt, ছamburg, Babeftr. 11.

Mithal, Julius, Megierungstat, Dureftor bes Bellengefängniffes, Rürnberg, Şürthet, ftrafe 114.

Dr. Midel, 3., Rotar, Dberbronn i. E.

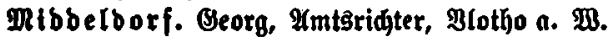

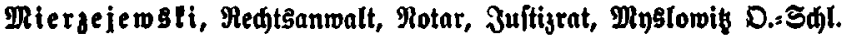

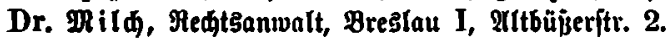


Dr. IRinben, Oeorg, Direltor bes \$janbbriefamtes, Berlin W. 62, Aleiftftr. 1.

Miquel, Imtsgeridtstat, Sarburg a. E.

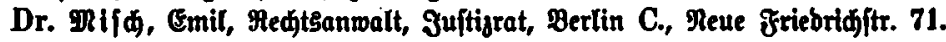

Dr. Mitteis, \&., Brofeffor, Reipgig, billerftr. 8.

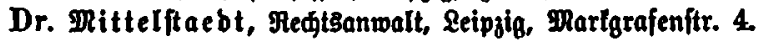

Dr. MRittelfte in, MRac, Obetlanbesgeridtšrat, ఏamburg, Slofterallee 49.

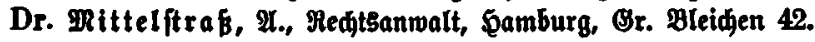

Dr. Mittermaier, 2 ., Profeflor, Eiefen, Riebigftr. 36.

Poedlinghoff, Medtisanmalt, Münfter $i$. B.

Robr, D., Medtisanmalt, Mubolftabt i. $\mathfrak{x h}$.

Dobrmann, Jujtizrat, Berben i. Sannover.

Dr. Moll, א., 3ujtigrat, Berlin W., Rarlsbab 26.

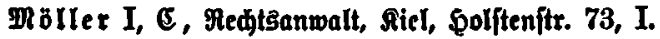

Moenniger, Carl, Direttor unb Sanbelsridter, Sarlsrube i. $\mathscr{B}$.

Morris, Georg, Redtt5anwalt, Berlin SW. 48, Jriebridftr. 16.

Mor\$bа丶, Medtsanwalt, Juftizrat, Bonn.

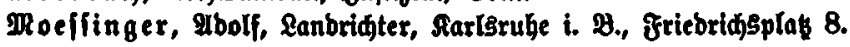

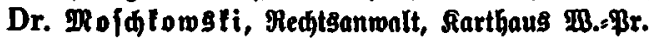

Dr. Mothes, Fubolf, Recttsanwalt, Leipjig, Purgitr. 35.

Motty, Mtedtsanwalt, Sufttzrat, Oräł (Pojen).

Mügel, Beh̆. Dberşuftizrat, ¿vortragenber Mat im Suftizminifterium, Berlin W. Surfürftenbamm 32.

Dr. פRü

Rübling, Staatsanwalt, Saristube i. $\mathbb{B}$.

Muller, Meferenbar, Berlin W. 50, Insbaderitt. 22.

Mi üller, Reфtşanwalt, Notar, Suftizrat, Demmin.

MRülter, \&., Redttanmalt, Motar, Ologau, Boltitr. 1.

Dr. IR ũ Iler, Ernft, Medtsanmalt, 3uftigrat, IReb, Ciplanaben 4.

DRüller, Iuguft, Fedteanwalt, Mannheim.

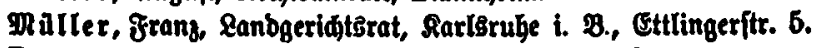

Dr. IRaller, A., Medtsanwalt, פReb, Esplanabenftr. 4.

IR üllers Erjba市, Brofeffor, Bonn, Bemusbergweg 32.

IRAlter, Eb., Reidsggeridtsrat, Reipzig, Elfterftr. 49.

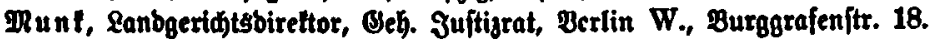

Dr. Mustat, Cugen, Imtsgeriđtsrat, Balbenburg i. S丸l.

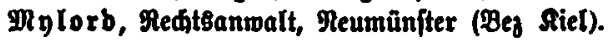

Matban, Medtbanwalt, Breslau I, Sdweibnizerftr. 37.

Dr. Mauen, Bictor, Rebtsanwalt, Mannbeim.

Raumann, C., Medtsanwalt, Rotax, Rüdow (Prov. Sannoder).

Redel, Ranbgeridti̊rat, Raristube i. $\mathscr{P}$.

Remnid, Ranbgeridtstat, Cöln a. Mh., Midjarb Magner[tr. 10.

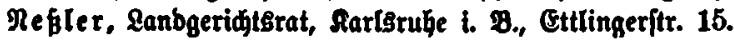

Reu, Fedhtsanwalt, IRez, Esplanabenftr. 12.

Reubauer, Senatspräfibent, Beq. Dberşuifijrat, Berlin W., \$ürburgers itrabe 3.

Dr. Reubeder, Pripatbojent an ber Iniderfitat, Berlin=Gbarlottenburg, Rants ftrafe 118/119.

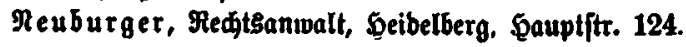


Neuburger, Redtianwalt, Baben:Baben.

Reuer, Milhelm, Medițanwalt, 3uftigrat, Cöln a. Mg., Langgaffe 23.

ReufeIb, Medteanmalt, Berlin W., Botsbamerftr. 29.

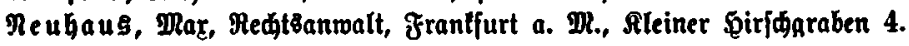

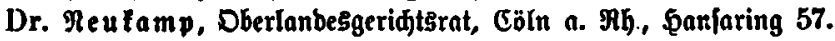

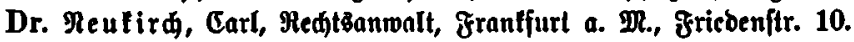

Dr. Reumann, Irmtsgeriatsrat, Preslau, Boetbeitr. 19, I.

Dr. Reumann, B., Sultizrat, Rotar, Breslau.

Re umann I, S., Redttanwalt, Rotar, 3uftizrat, Rerlin S., Cranienftr. 142.

Reumann, Itb., Juftizrat, Sorau (Niederlaufib).

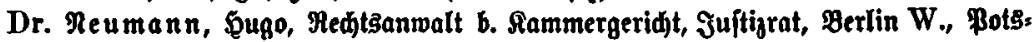
bamerftr. 118.

Dr. Reumann, Ernft, Referenbar, Berlin W., Dörnbergitt. 6.

Dr. Reumann, Staatsanwalt, Nadjen.

Me ufd, Redtsanwalt, Bleimit.

Niebner, Mlcranbex, Oberlanbeşgeridtšat, Ricl, Rinbenallee 19.

Dr. Riemeyer, Profeffor, Riel.

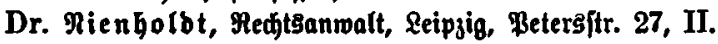

Dr. Riefe, Dragiftrat:alfeffor, Berlin NW., Bodumeritr. 21.

Niefe, Meditsanmalt, Notar, 3uftizrat, Riel, Solftenftr. 79.

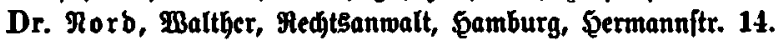

Dr. Morbmann, Redtsanwalt, MRülhaulen i. E., Grabenftr. 60.

Mowoczyn, Juftizrat, Neuftabt $\$$.s\$r.

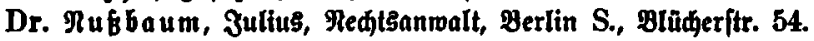

Dr. Dberned, Redtsanwalt, Rotar, 3uftizrat, Berlin W. 10, von ber Segotftr. 8.

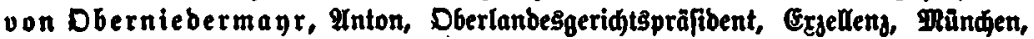
Mag̨mülleritr. 23, I.

Db redt, OS., Juftigrat, DRet, Bantitrafe.

Dehlert, Я., Dberlanbesgeridt Brat, Breibrüden.

Dr. Dehme, D. \&̊., Beh. Juftiłrat, Reipjig.

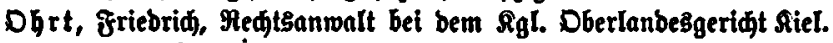

DIbridt, Erfter Ṡtaatsanmalt, \&ūneburg.

Dr. Delengeing, Nedtsanwalt, Mannbeim.

Dr. Delsner, 3uftizrat, Redtsanmalt, Notar, Franffurt a. MR., Reue Mrainzerfir. 68.

Dllenborff, Hedtsanwalt, BresIau, 3mingervlat 2.

OItmann, P., Redtéanwalt, Sujtizrat, Breifşwalb.

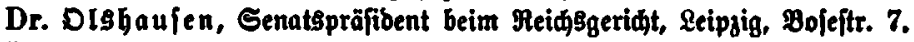

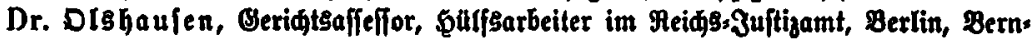
burgeritr. 31, II.

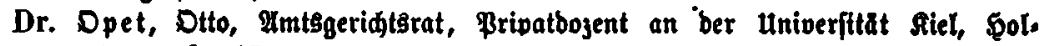
tenauerftr. 85.

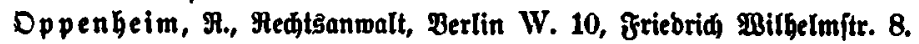

Dr. Dppenbeim, \&., Redtsanmalt, Juftizrat, Maing. Raijerftr. 18.

Dr. Dppenheimer, Paul, Amtsridter, Altona.

Dr. Dppenbeimer, F., Medtsanwalt, 3uftijrat, Berlin W., Maueritr. 34.

Dr. Dppen heimer, 3., Redtsanwalt, Zranffurt a. MR., Bötheitr. 2

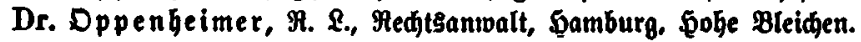

Dppenteimer, Salomon, Hedtsanwalt, Sarisrulge i. P., Rreujitr. 8. 
Dr. Dppermann, 20, Affefior, Itnnaberg i. Є., Benersborforitr. 4.

Dr. Dppermann, Reidjgeridit3rat, Leipsig, Egelitr. 5.

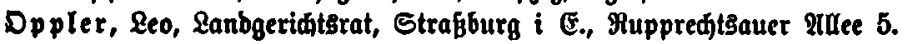

Drgler, Rurt, Ftedtsanwalt, Barmen.

Dr. Drgler, Mechtsanmalt, Pofen.

Dr. Drtlieb, Geridtsaffefior, Sdjöneberg, Martburgftr. 29.

Dr. Dertmann, Baul, Brofeffor, ErIangen.

Dr. jur. Ofterrieth, Brofelfor, Єdriftiteller, Berlin W., Bilfelmitr. 57/58.

Dr. Dswalt, Senry, Juftizrat, Frantfurt a. Mr., Bodenheimer Lanbitr. 2.

Dr. von Dtto, Biftor, Juftigminifter, Dreకben, Bürgerwieje 25.

Dr. v. Dverbed, I., Frciferr, Profefior ber Redte, Freiburg $\mathfrak{i}$. Edweiz.

Dr. \$ab ft, Imtsridter, \&ubed, fricorid झBilgelmitr. 40.

Padffier, Med)tsanmalt, Berlin W., Surfürítenbamm 29.

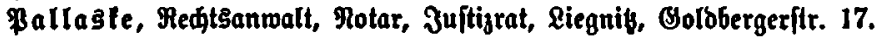

Dr. Panther, Redjtsanwalt, Mannbeim.

Dr. Pape, Ernit, Snmmergerifterat, Preslau, Bölhcitr. 45/7.

Dr. Pappenheim, Max. Profeffor, Fiel, Moltteftr. 57.

Dr. jur. et phil. Baffor, Mid., Referenbar, Brivatbogent, Frantjurt a. MP., Rlemensîtr. 4.

\$auli, Emil, Lanbgeridtstat, Berlin NW. 87, Iltts Dloabit 73.

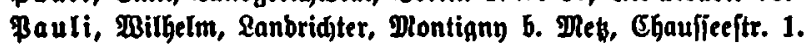

Freiberr von Đedmann, M., Direttor ber Bayerijden Sanbetsbant. DRündjen, Daffiftr. 5, II.

Peifer, Deintid, Lambgetidgtsrat, Dangig.

Beltafohn, Bruno, Medtsanmalt, Berlin W. 15, Fajanenjtr. 59.

Dr. Berels, Leopolt, Pribatbojent, Beridts:2tfiefior, Seibelberg, Bergitr. 4.

Dr. jur. Perels, Curt, Privatbojent an ber Univerfität Riel, ફoltenauer[tr. 139.

Berßament, Midjael, Brofefior, St. Petersburb, Waffili Dftroms:Sinie 10, Saus 41.

Dr. \$erl, Đeditsanmalt, 3uftizrat, Berlin W., Surfürftenftr. 89.

Dr. von Beftalozza, Unton, Oraf, Münden, Bring Megentenplay 23.

Beter

Dr. Peters, Reidjsperidjtstat, Leipjiß, Beethovenjtr. 14.

Beter $\$$, Norbert, Redtzanwalt. Coblens, \&öbritr. 31.

Dr. Beterien, Ghr., Medjtsanwalt, Motar, Flensburg.

Betrid, Medtsanwalt, Notar, 3uftigrat, Samter.

Beball, Bertholb, Redtsanwalt, Berlin C., Neuc \&riebridjitr. 21.

Dr. Bfefferle, Geridtsaffeffor, Sarlsrube i. :., Renfftr. 10.

Bfetffer, Dberlanbesgeridtstat, Stettin, Sarfutidieftr. 2, I.

Dr. Pfersborff, Zrib, RehtBanwalt, Straßburg i. E.. Eifennermannspla 4.

Pfibner, Beorg, Eanbridter, Grantfurt a. PR., Dber:Ylgemer 4.

Dr. Pflũger, Profefior, Bonn, Raifer Friebridftr. 7.

\$hillip pi, Meditsanwalt, Dles, Bäreniftr. 4.

Dr. PGilipps6orn, Referenbar, Berlin W. 15, \&ajanenftr. 22.

Pid, IImtsridter, Berlin 0. 112, Finoweritr. 20, II.

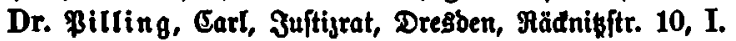

\$incus II, Eeorg, Redtsanwalt, Juftijrat, Berlin N., Unter ben Linben 41.

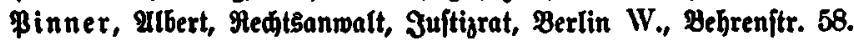

Dr. Piper, \&anogeridjtspräfibent, Reujtreliţ, Seeitr. 32. 


\section{XIJII}

Piftor ius, Redtsanmalt. Magbeburg, Breiteweg.

Dr. Bland, Birflider Bebeimer Mat, Brofeffor, Erzellenz, Eöttingen.

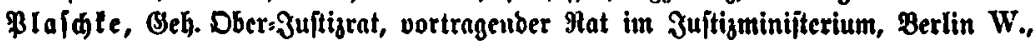
Sdjaperitr. 22.

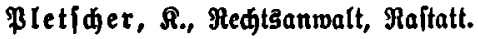

Blonster, Redtsanmalt, Rotar, Berlin N., Babftr. 60.

Dr. Polzin, Meferenbar, Gannoder, (Sretdjenitr. 42.

Dr. Bomme, ßrivatgelehrter, Berlin W. 30, Seilbronnerftr. 31.

Bomorsfi, Hedtsanmalt, Suftizrat, Ditromo.

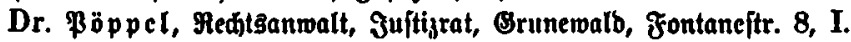

Bort, Juftizrat, Colmar i. E.

Bofner, Gerrmann, Medttsanmalt, Berlin W. 66, Wilgelmitr. 88.

Dr. Bofener, Baul, 2lffeffor, CGarlottenburg, Bleibtreu[tr. 18.

Dr. Pralle, R., Medtsanwalt, Notar, Bremen.

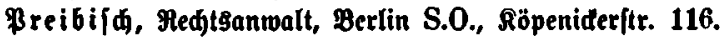

PreißB, Medtsanmalt, Tarnomik.

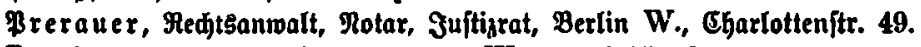

Dr. Preū́, \$., Privatbojent. Berlin W., Drattbäifirdjitr. 29.

Briefter, Đarry, Rectsanmalt, Berlin S.W., Bimmerftr. 21.

von Brittwits unb \&affron, Dberitaatsanwalt, Beh. Ober.Jultizrat, Naums burg a. 5 .

Proţen, Geh. Sujtizrat, Amts̈geridtsarat, Matibor.

Prym, Emil, Medtsanwalt, Düren i. Mgld., Sübftr. 13.

Dr. Pubel, Redtianmalt, Pannhein.

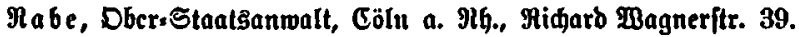

Dr. Mabel, Ermit, Unid., \$rofeffor, Bajcl, Uretlingerftr. 84.

Mabenhofer, Jofeph, Dbers\&anbesgeridtsrat, MRünden, Sonnenftr. 3.

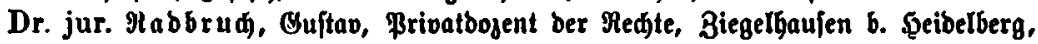
Billa Stiftsmmühle.

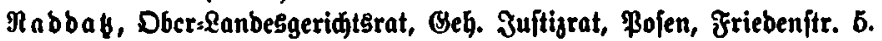

Mablauer, Amtşgerid)tsrat, Đulle a. b. S., Martinsberg.

Dr. jur. Mafdte, Marie, Or.sLidterfelbe $\mathbb{B}$ eft, Sternftr. 17 a.

Dr. Mathenau, frib, Eanbridter, Berlin W., Mcinedeftr. 7.

Mathje, Sermann, Neditsanwalt, Siel.

It u, PReinlolb, Redttsanwalt, Pünden, Ranbwebrftr. 4.

Dr. Maud, Carl, Profeffor, Sönigsberg i. Pr.

Dr. jur. Mauert, Niel, Zeloftr. 81, I.

Ra umann, Dstar, Reatsanwalt, Rotar, Berlin N., Chauffeeftr. 131.

Ređ, Lanbgeridtspräjiben\}, (Sseh. Ober-Şuftijrat, Sedjingen, Sohenjollern.

Dr. Negelsberger, Behcimrat, Profeffor, Bbttingen.

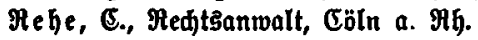

Dr. MeGm, E., Brofeffor, Strafburg i. E., Đohenlohejtr. 22.

Dr. Mehme, \$aul, \$rofeifor ber Medte, \$alle a. D. S.

Dr. Meidarbt, Molf, Mejeŕendar, Reipjig, Sottjđebjtr. 19, III.

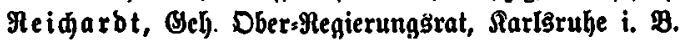

Dr. Reide, Martin, Reditsanwalt, Juítizrat, Berlin S.W., Sraujenitr. 42/43.

Reidjenbad, Redjtsantwalt, Berlin N.58, Sdjönjaujer Aruee 50.

Dr. Reidiert, Juftizrat, Sannover.

Meidholb, ₹., Medtsanwalt, Lanbau i. PFalj. 


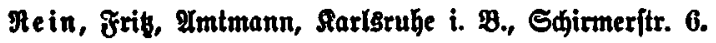

Dr. Feinad, Sujtizrat, Mülhaujen i. Eljaß, Sinneftr. 16.

Meinberger, Međtsanmalt, Suftizrat, Berlin C., Breiteitr. 11.

Meinele, Redtsanwalt, ఏagen.

Meinharbt, Mirtlider Eebeimer Mat, Erzellenz, Sarleruhe i. $\mathfrak{B}$.

Meinmuth, 2., Mectsanwalt, Mannbein $\mathfrak{A} 4,5$.

Dr. Meis, ఆuitad, Mecttanwalt, Mannheim.

Meis, \$., Staatsanmaltidaftsrat a. D., Danzig, Sunbegafie 93.

Meifert, Juftizrat, Erfurt.

Meî, Baul, Suftizrat, ̧rantfurt a. Dr., Mejerftr. 51.

Meitinger, Th., Borftanb bes Grunbbudjants, Medtstat, Mannbeim, Manns beimerft. 51.

Dr. Menanb, Medtsanwalt, Colmar t. E.

Menner, Iuguft, Oberamtsriditer, Maftatt.

Meufder, Medtsanwalt, Motar, Juftigrat, Sottbus.

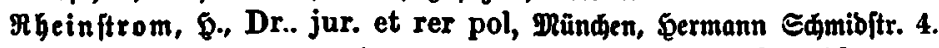

MGeinwalb, Dberlanbeşgeridtstat a. D., Stuttgart, Redarftr. 48 b.

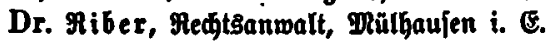

Miede, Redtsanmalt, פalle a. S.

Midter, Carl, Rlbredt, Referenbar, Leipjig:Connemik, Cübitr. 108.

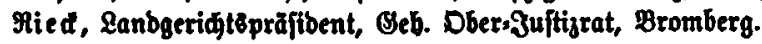

Riebel, Sigmunb, t. Amtsriater, frordheim i. 9 .

Dr. Riebel, Dberlanbesgeridterat, Raumburg a. b. S.

Dr. Miemen[dneiber, Medtsanwalt, Notar, Bremerhaven.

Dr. Riefenfelb, Conrab Grnit, Snnbifus ber Sanbelstammer, Geriatsafieffor a. D.,

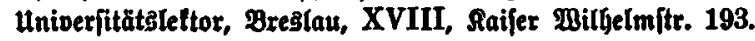

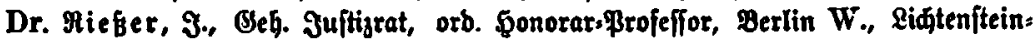
ITtee 4.

Dr. Miet\{del, Siegfrieb, univ.॰\$rofeffor, Tübingen, Redarbalbe 55.

Dr. Riegler, E., Profeffor, Freiburg i. :., Boetgeftr. 15.

Ming, Rammergeridtstat, Berlin W., Sdöneberger ujer 46.

Dr. Fintelen, MRaz;, \$rivatbogent, \&eiplig, Sibonienftr. 68.

Mintelen, Medtsanwalt, \$agen i. $\mathfrak{B}$., \$oditr. 74 a.

Mippner, M., Mectsanwalt, Juftijrat, Berlin SW. 48, Friebridfitr. 235.

Dr. Hitter, Erwin, Obersilmtsridter, Marlšrube i. $\mathscr{8}$.

Dr. Mitthau|en, Mechtsanwalt, Berlin NW. 6, Souijenitr. 39.

Dr. Mobinow, M., Samburg, Reuerwall 69.

Moebiger, Medtanmalt, Mannheim.

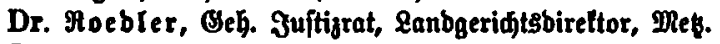

Dr. v. Robland, 28., Geh. \$ofrat, Brofeffor, Jreiburg i. Baben, Goetheitr. 28.

Röhrig, Medtşanwalt, Berlin W., Eharlottenjtr. 62.

Moll, Redtşanwalt unb Rotar, Rifia i. .

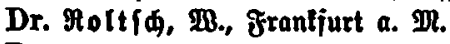

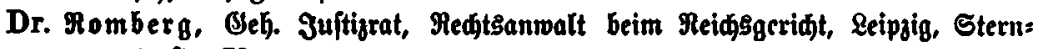
martenftr. 79.

Dr. Moemer, Mectsanwalt, Rotar, Juftigrat, Bođum i. $\mathfrak{B}$.

Monniger, P., Reditsanmalt, Reipjig, Peterŝttr. 27.

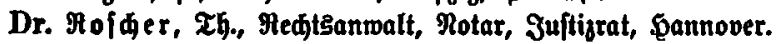




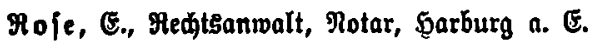

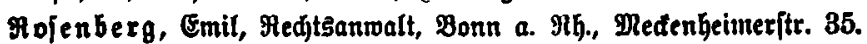

Dr. Noienberg, Nedtsanwalt, Siegen.

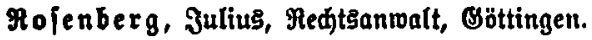

Dr. Rojenberg, Reo, Beridts=2lffeffor, Privatbojent a. b. Univerjität Osöttingen.

Ro/enberg, Ober:arbesgeridtstrat, Eolmar i. E.

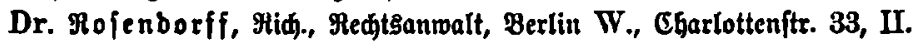

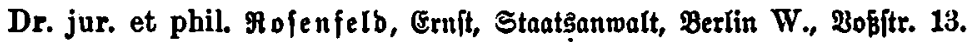

Dr. Mofenfelb, E. G., Profeffor, Müniter i. $\mathfrak{B}$, Aleimannitr. 3.

Dr. Mofenfels, F., Mecthtsanwalt, Mannheim.

Dr. Mojenfelb, 3., Reditsanwalt, Mannbeim.

Mofenmeyex, Redttsanwalt, frantfurt a. M., Goetheftr. 35.

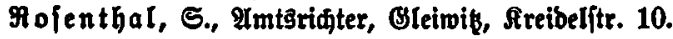

Molenthal, Walter, Medtaanualt, Berlin SW., Sinbenjtr. 112.

Dr. Mofenthal, Milhelm, Fedtsanwalt, Münden, Füritenfelderitr. 10.

Dr. Mofin, Beh̆. Đofrat, Brofefior, Zreiburg i. Baben, Bünthersthal 64.

Dr. Moth, Mriegsgeriatstat, Dregsen $\mathscr{A} .$, Elijenjtr. 11.

Dr. Moth, Redtsanwalt, Seibelberg, Theateritr. 14.

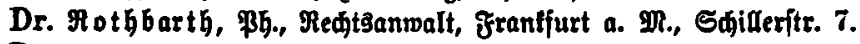

Dr. Mothe, Jujtizrat, Zerlin W., Potsbameritr. 24.

Motbe, Curt, Medtsanwalt, Ehemniz, Theateritr. 86, II.

Motbidilb, Redtsanwalt, Brudjal, Sdilleritr. 10.

Motbíillb, Meditspraltifant, Seibelberg.

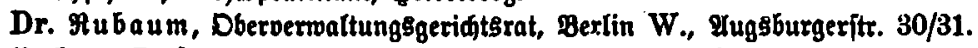

Ruben, Ernit, Beriftssteffeffor, Berlin, Orolmanitr. 34/5.

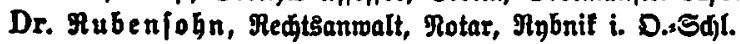

Dr Mubmann, Staatsanmalt, Bforbbeim.

Rübe, Motariatsinfpeltor, Farlsmbe i. 8 .

Dr. Mubolph, Juftizrat, ডannover, $\mathfrak{A m}$ Sđjiffgraben 61.

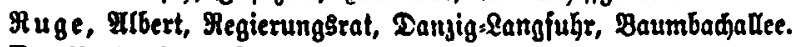

Dr. Mulanb, Suftizrat, Colmar i. E.

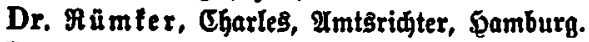

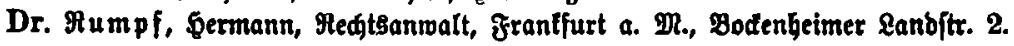

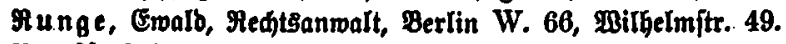

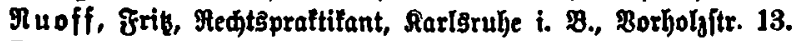

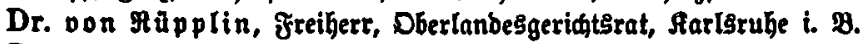

Dr. Saalfelb, 2ulfreb, Medttanmalt, Berlin C., Mojentbalerftr. 18.

Saafen, 3. S., Notar, Bonn, Raiferplay 5.

Saфs, Emil, Oeh. Suftigrat, Leipzig.

Dr. jur. Sad, Th., Sdbrift|teller, QRündien, Frang Joịphftr. 20.

Salinger, Max, Juftizrat, Redtsanwalt, Notar, Berlin W., Sdjellingitr. 16.

Dr. Salinger, Beorge, FedtSanmalt, Berlin W. 15, Jaaanenftr. 67.

Salinger, Mecttsanwalt, Rotar, Mollítein.

Dr. v. Salis, Profeffor, Sdló Maridlins b. \&anbquart i. Oraubünben, Sđmeiz.

Salomon, Sanbgeridgtsrat, Charlottenburg, Siejebredititr. 10.

Salomon, Ulfreb, Ranbgeridjtsrat, Cöln a. Rh., Bolfsgartenftr. 24.

Dr. Salomon, 3. Ph., Međtşanwalt, Berlin W. 35, \&üţomitr. 67. 


\section{XLVI}

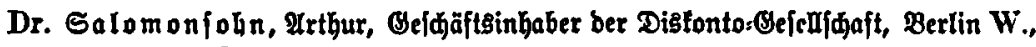
Tiergartenfitr. 8.

$\mathrm{Sal}_{3}$, Meditsanmalt, Juftizrat, Bojen.

Dr. Samfon, 5., Redtsanwalt, ডamburg, Bleiđenbrüde 10.

Dr. Samfon, $\mathfrak{V}$., Samburg, Reucr $\mathbb{M a l l}$ 19/:3.

Dr. Samter, Sermann, Juftizrat, Berlin W., Dohrenitr. 65.

Dr. Sammer, R., Beh. Megierungstat, Bantbireftor, Botba, Dhrbruferftr. 4.

Sanbberg, Ouftav, Medtsanwalt, Juftizrat, Berlin S., Dranienftr. 45.

Dr. Sanber, E., Mechtsanmalt. Berlin W. 62, Rutheritr. 13.

Dr. Sanbers, Redtsanmalt, Sarls̈ruhe i. B., Sebelitr. 9.

Sänger, A., Mechtganwalt, Stuttgart.

Dr. Sartorius, Brofeffor, Greifsmalo.

Saul, 3mmanuel, RedtEanwalt, Duisburg, Rönigitr. 38.

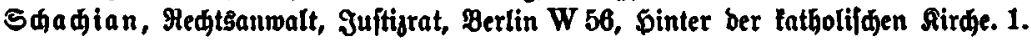

Dr. Sळ) a te I, \&co, Reditsanwalt, Berlin SW., Wilbelmftr. 100.

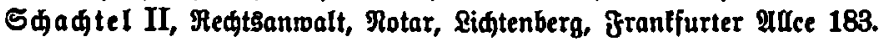

Sdaefer, 2luguft, Fedtsanwalt, Babens:Baben, Sophienftr. 29.

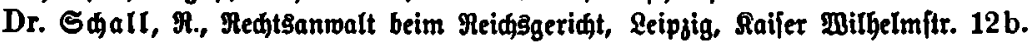

Dr. Sqanje, Ostar, Brofeffor, Raiierl. Megierungsrat a. D., DreSben, Jacobiftr 5.

Dr. S由аrlađ, 3uftizrat, Strafburg i. E., Blauwolfengafie 8.

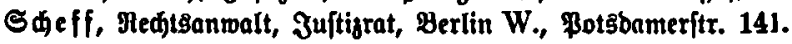

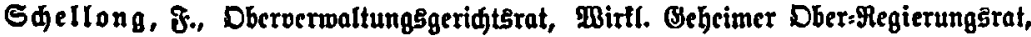
Eharlottenburg, Earmeritr. 10.

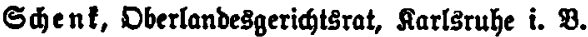

Sdend, Medtsanmalt, Бapen i. $2 .$, Bergitr. 73.

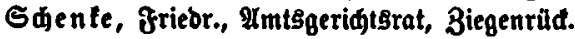

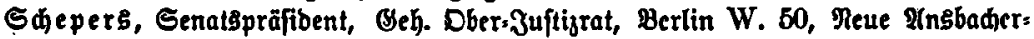
Ptrabe 13.

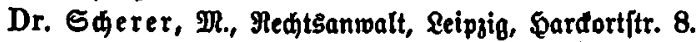

Dr. Sderer, Dtto, Medtsanwalt, PRainz, Saiferitr. 46.

Sdippanß, Erid, Lanbridter, Sicl, Bejelcr \&uce 32, I.

Sdirxen, Medg1sanwalt, Sujtizrat, Motar, Ficl, Flinfe 26.

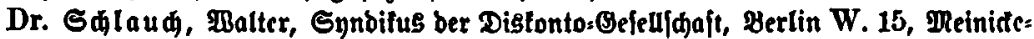
ftrafe 2.

Dr. jur. Sळlegelberger, Lanbridter, $\&$ yd $D .: \$ r$.

Sdlefinger, Georg, Redtsanwalt, Reipjig, Reumart 1.

Sqlidt, Redtsanmalt, Rotar, Soegel (\$roo. (jannover).

Sdlodermann, Eanogeridtsbirettor, Berlin W. 15, Nadjobjtr. 18.

Sidloß, Redtsanmalt, Seilbronn.

Dr. Sфlofmann, Profeffor, Sitel.

Dr. Sฌlotter, Lanbgeridtstrat, Magbeburg, Iugujtaftr. 27.

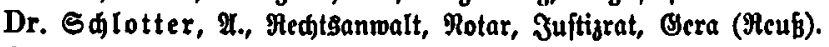

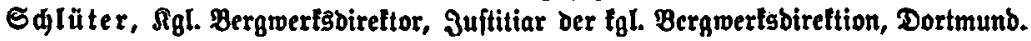

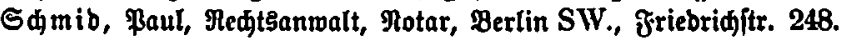

Sdmib, Senatspräjibent, Beh. Dberşuftijrat, Siel, Eorenłenbamm 6, II.

von Sdmiolin, Staatsminifter ber Sultiz, Stuttgart.

Dr. Sdmibt, Arthur, Brofefior ber Med,te, Beh. Juftizrat, Giepen, \&ontyftr. 18.

Sdinibt, Mubolf, Landgeridtstat, Starlsrube i. 2 . 


\section{XI,VII}

Sdmibt, Beridtsaffefijor, Synbifus ber Norbbeutiden Brauervereinigung, Detmolb. Sdmibt=28arbeleben, Rammergeridt3rat, Berlin SW., Rleinberenitr. 27, III. Dr. Sфmibt, Midarb, (Beh). bofrat, ßrofeffor, Freiburg i. 8.

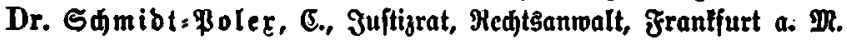

Dr. Єdmibt, Carl, 3uftizrat Mtedtsanmalt, foüth (Bayern).

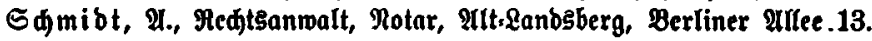

Dr. Єdmiot, Ranogeriditకrat, Sartšrube i. $\mathfrak{B}$.

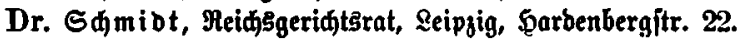

Sdmibt, \$., Sgl. Lanbgeridtstat, Deggentorf.

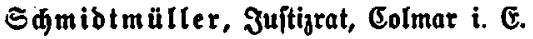

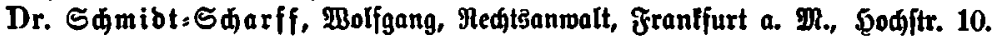

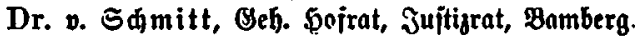

Sdmi

Dr. Sđmił, Berharb, 3uftizrat, tgl. Rotar, Düffeloorf, Steinftr. 28.

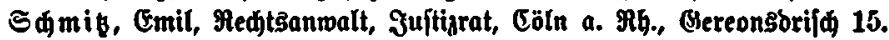

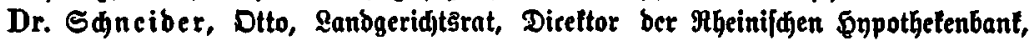
Danntheim.

Eqneiber, Amtggeridtsarat, $\Re 0$ [cn, Rönigsplą 5.

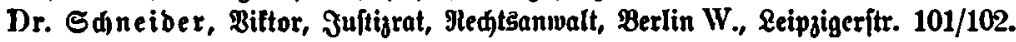

Sdneiber, Irnolb, Medtsanmalt, Motar, Betlin W. 8, Griebridftr. 83.

Sdneiber, Johannes, Redtsanwalt, Meerane i. S.

Edynitger, 3., Red)tsanwalt, Sultizrat, Detmolb.

Dr. Sdnizler, Medteanwalt, Stefelb, Dftwall 149.

Dr. Sđjolz, Dberverwaltungsgeridtstat, Berlin W., Manfeitr. 13.

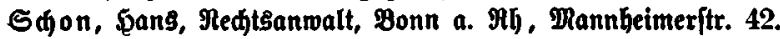

Sqbenfelb, Rechtsanmalt, Berlin C., Rönigitr. 5:.

Edoenlant, bugo, Medtsanwalt, Notar, Tegel.

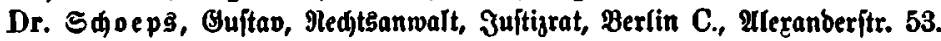

Sdordt. Juftizrat, Đameln a. b. פ⿹ejer.

SqDetenfad, Qugujt, Privatbogent, Bürzburg.

Dr. Sdott, Profefior, BresIau XVI.

Sdjöttlänber, Redttsnnwalt, Pofen.

Sdraber, א., Eifenbahnbireftor a. D., Mitglieb bes Meidstags, Berlin, Steglizer: jtraje 68.

Ed)raber, Rectsammalt Diebentjojen.

Dr. Sdrag, Redjtsanwalt, Rarle̊ruhe i. :., Malbftr. 41.

હdjeiber, Iluguft, Nedtşanwalt, Donauejdingen.

Sdreier, Dsfar, Mechtsanwalt, Berlin C., Reue Promenabe 3.

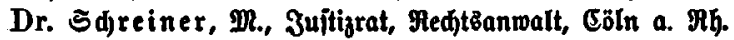

Dr. Sđrod, E., Redtsanwalt, Sultigrat, Marienwerber $i$. Meftpreußen.

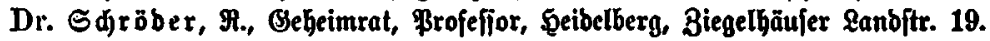

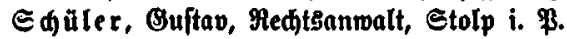

Saulk, Ernft, Nedjtsanwalt, fgl. Notar, Fillehne (ßojen).

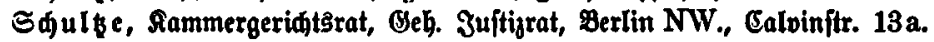

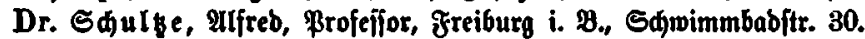

Dr. Sфulłenftein, Senatspräfibent bes Dberverwaltungggeridts, Berlin W., PRoø̧itr. 86.

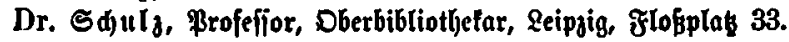




\section{XLVIII}

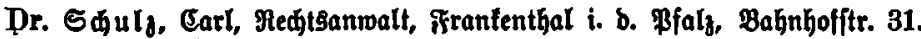

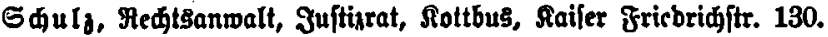

von 5 น $l_{8}$, Mlagiftratsrat, Berlin NW. 52, Thomafiusftr. 21.

Dr. Sdul

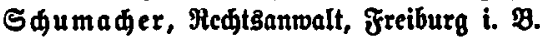

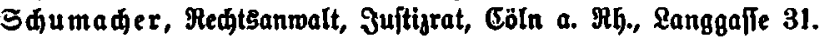

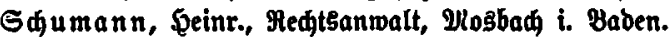

S๙)umann, Staatsanwalt, Bolen.

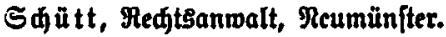

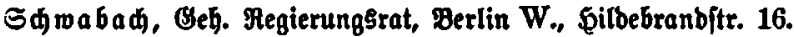

Dr. Sфmar

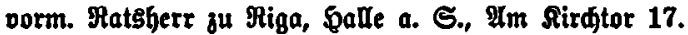

Dr. S中warße, Dr., Lanbridtex, 5amburg, Loogeiteg 19.

Dr. jur. Sdwarłfopff, 3., Dajor a. D., IImt3ridter, Meftenb, Eidenallee 33.

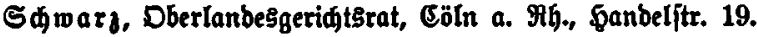

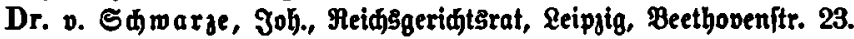

Sđweidler, Staatsanwalt a. D., \&übed, Babnjtr. 2.

Sdweidert, Carl, Redtsanwalt, Frantenthal i. b. Pfald.

Dr. Sdweiłer, Mbolf, Geridtsaffeffor, Straßburg i. E., Blaumoltengaffe 7.

Sawelger, \$., Medtsanmalt, Straßburg i. E.

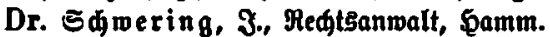

Sawoerer, Staatsanwalt, Sonjtan i. $\mathfrak{B}$.

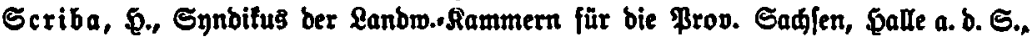
uleftr. 8.

Dr. Sedel, E., ßrofeffor, Berfin W. 62, Rurfürftenitr. 76/77.

Dr. Sée, Ebgarb, Qrodolat, \$aris, 4 rue le Peletier.

Seeger, 21., Remtsanwalt, Straßburg i. E., Steinftr. 1.

Dr. v. Seeler, Milh., Brofeffor, Berlin W., Ildenbaditr. 2.

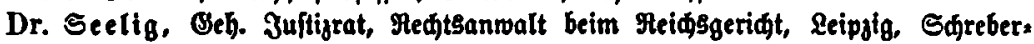
itraje 13.

Dr. Seelig, Mectisanwalt, Berlin W., PotSbameritr. 9.

Dr. Seelig, Lubrig, Redtsanwalt, MRannheim.

Dr. Seeligmann, Medtsanmalt, Farls̈ruhe i. P., Rönigftr. 108.

Dr. Segalla, Fledtsanwalt, \$amburg.

Seibert, Midter, Bremen, Bismardftr. 71.

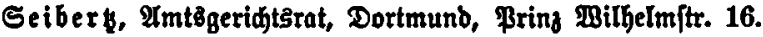

Dr. Seiler, Dtto, Medtsanmalt, MRannheim.

Dr. Seił, Carl, Jofef, Eanbgeridtsrat a. D., Deünden, Bruberitr. 1, I.

Dr. Selb, Emil, Redtsanwalt, Dannbeim.

Selb, B., Redtts= unb fistalsilnwalt, Mannbeim, A. 3. 9.

Seld, Erwin, Geridtereferenbar, Grantjurt a. In.: Sadjienbaufen, Darmftäbter. Ianbitr. 92.

Seli 8, Qeh. 3uftizrat, \$anbsbed.

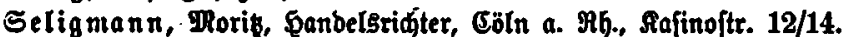

Dr. Seligfohn 1, Arnolb, Suftizrat, Medftsanmalt, Berlin NW., Pring \&ouis Gerbinanbftr. 1.

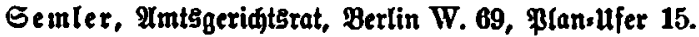

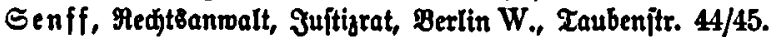




\section{XLIX}

S̄enger, Mrac, Pedtţanwalt, Graubenz.

Senninger, 3ofef, Redttzanwalt, Baffau, Rubrigitr. 285.

Seyfert, E. S., Senatspräfibent, Dresben, Stephanien/tr. 30.

Dr. jur., Sibi, 3., Thünhen, Datbilbenjtr. 12, 11.

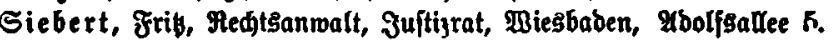

Dr. Siede, Mectisanmalt, Reipzig, Thomastirdgof 17.

Siegel I, Reopolb, গtedtşanwalt, Juftizrat, DhünЊ̧en, Dieneritr. 20.

Dr. Sieger, $\mathfrak{~}$, Nedttsanmalt, Notar, \&rantjurt a. $\mathfrak{D}$.

Sieghoff, C., Redtşanmalt, इeibelberg, Scauptftr. 124.

Dr. Siegmann, Eeorg, Medtsanmalt, Berlin SW., Einbenitr. 37.

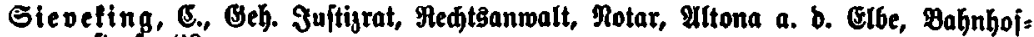
ftrape 20.

Dr. Silberidjmibt, Dberlanbesggeridtstrat, Bmeibrüđen.

Dr. Silbe rite in, Max, Med)tsanwalt, Suftizrat, Berlin, Margarethenitr. 7.

Dr. Siméon, \$., Sammergexidłţrat, Wilmersborf, Eijenjabnjtr. 44.

Dr. Simon, \$. B., Jujtijrat, Nechtsanwalt, Berlin W., Biftoriaftr. 5.

Dr. Simon, Sang, Neditsanmalt, Berlin W., \&üzomitr. 82.

Dr. Simon, Dtto, Redtsanwalt, Mannheim.

Dr. Simonis, 2lbolf, Bürgermeifter, Moltod $\mathfrak{i}$. Medlenburg.

Simons, W., Lanbridter, \&r. Lidterfelbe.WBeft, Steglikerftr. 39.

Simonfon, Oberlanbesgeridtsrat, Brestau, Sinbenallee 6.

von Simfon, Beh. 3ujtizrat, Reditsanwalt, Rotar, Berlin W., 3äger[tr. 52.

Sinn, P., Reditsanwalt und Notar, 3uftizrat, Mendsburg.

Singheimer, Feđtsanmalt, Orünftabt.

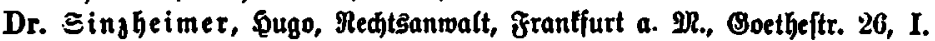

Dr. jur. Slugemsti, Emil, Snnbifus ber 2IIgemeinen EleltrijitätSgefellifajt, Charlottenburg, Leibnibit:. 73.

Slugemsti, Rechtsanwalt, Berlin C., Maúftr. 3.

Smolla, Redtsanwalt, Rotar, Milfter i. \$.

Dr. Sobernheim, Dtto, Referenbar, Berlin, Mllenftr. 3a.

Dr. Sololowsti, Brofeffor, Rönigsberg i. Br.

Solban, Gans, Mechtsanwalt, Maing, Stabionerbofftr. 2.

Dr. Solmifen, Georg, Geriळtsaffeffor, Direttor b. Distontogefellijaft, Betlin W., unter ben Sinben 35.

Sonnenfelb, \$., Redtsanwalt, Berlin, Reipjigerftr. 108.

Dr. Sontag, IImtsridtcr, Sattowił, Leffingitr. 9.

Dr. Spetber, Eeneralfetretär bes beutjden \$anbelstages, \$annjee, \&ohengrin: ftrafie 28.

Dr. Spałn, Dberlanbes̊geridłțpräfibent, Riel.

Spangenberg, Medttsanmalt, Rotar, Jujtizrat, Sannover, Beorgitr. 13.

Dr. jur. Sperling, Geinrid, Lanbgeridtspräfibent, \&yd, D.s\$r.

Dr. Spiegel, פ., Hedtsanwalt, Mannheim.

Spießel, खilhelm, Redtsanwalt, Riel, Fleetbörn 1/3.

Dr. Spittel, Redtsanwalt, Rottbus, Raijer Friebriditt. 1.

Dr. Spohril, Medtsanwalt, Oiejen.

Springe, \$erner, Redțanwalt, đIttona a. E., Brofie Bergftrafe.

Springer, Frik, Meferenbar, Eharlottenburg, \$arbenbergitr. 10.

Staebel, E., Redtisnnwalt, Offenbad a. M.

Dr. Stabthagen, 3ul., Juftizrat, Notar, Berlin SW., Bimmeritr. 16/17. 


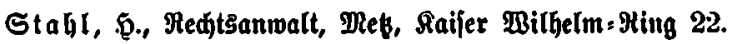

Stahn, Eugen, Amtsgeriđtşrat, Breslau, Brandenburgftr. 5\%.

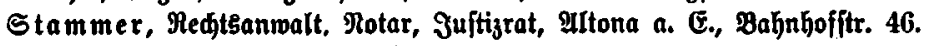

Stapif, A., Mechtżanwalt, 3ultigrat, æeimar.

Starle, B., Sujtizrat, Meiba b. Beta.

Dr. Stauber I, Rarl, Med,tsanwalt, Rürnberg, Dbitgajie 2.

Dr. Sta $\mathfrak{i}$, Medtsanmalt, Bmidau i. S.

Di. Etebens, Redtsanwalt, Rotar, 3ujtijrat, Bertin W. 8, Gharlottenitr. 57, Ш.

Steffan, \&anogeridgtsrat, Straßburg i. Elf., Ipffeliftr. 4.

Dr. Stein, Brofeffor, Reipjib, Ferbinand Mgobejtr. 28.

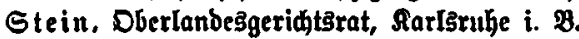

Dr. Stein, Recttsanmalt, Bantier, Sarlšrube i. P., Seminarjtr. 15.

Steinel, Eugen, Medtsanwalt, Pforgheim.

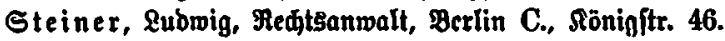

Dr. Steiniz, Medtsanwalt, Sujtijrat, Berlin SW., Sommanbantenftr. 72.

Dr. Steiniß, Max, Medtsanwalt, Berlin N. 54, Brunnenjtr. 25.

Dr. Steiniß̧, Curt, Redjtsanmalt, Breslau, Saijer Bilbelm[tr. 57.

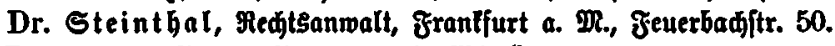

Dr. Stern, Bruno, Medt\&antwalt, Mürburg.

Dr. Stern, 3acques, Beridtsaffeffor, Berlin C. 25, भIfezanberftr. 63.

Stern, Dax, Obersanbeşgeridtsrat, Düffelborf, Felbftr. 12.

Dr. Stern, Dtto, Sanbridter, Beuthen.

Dr. Stern, febor, Juftizrat, Berlin W, Behrenftr. 23.

Dr. Stern, S., Redtsanwalt, Dlannheim.

Sternberg, Ranbgeridtsrat, Charlottenburg, Grolmanftr. 6.

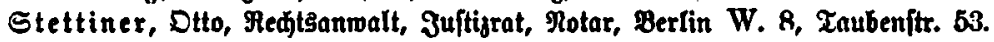

Stenrer, Frib, Direltor ber Bayer. Sandel\$bant, Münden, Maria Therefiaftr. 13.

Dr. Stiers Somlo, Unio.s Profefior, Bonm, Sronpringenftr. 3.

Stillfdweig, Rectsanwalt, Berlin W. 0, Botgbamerftr. 141.

Dr. Stinłig, M., Profeffor, Reipjig, Sibonienftr. 67.

Dr. Stoedel, Reidsgeriळtstat, Leipjis, Sebanjtr. 1.

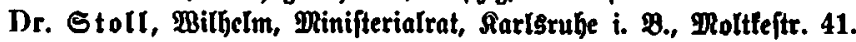

1)r. Stolje, Mhagiftrats:2lffeffor, Obarlottenburg, Sdjulftr. 14.

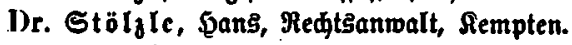

Etory, Mlfreb, Medtianwalt, Berlin S. 42, Dranienftr. 145/46.

Storj, Dberlandesgeridtsrat, Sartsruke i. $\mathfrak{B}$.

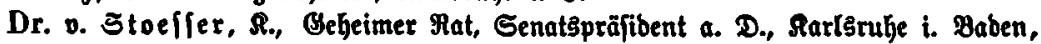
Jabnitr. 6.

Straegler, Rammergeridtstrat, Berlin W., Geisbergitr. 12/13.

Dr. Stranz, 3., 3ufitizrat. Berlin W., Reipzigeritr. 34.

Dr. Stranj, DR., Redtsarwalt, Berlin W. 62, Aurfüritenftr. 73.

Straffer, F., R. Oberamtsridter, Bab Meidenhall.

Straub, Siegfried, Medttsprattifant, Freiburg i. Br., Sarthäuferjtr. 40 III.

Straube, Ranogeridjtìrat, Galberitabt, Spiegelftr.

Dr. Straus, Morił, Medtsanmalt, Rarlsrube i. 2., Saijer[t. 712.

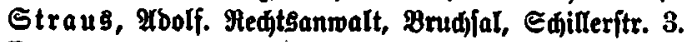

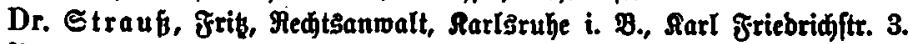

1)r. St ra úp, Mechtsanwalt, Dannbcim. 


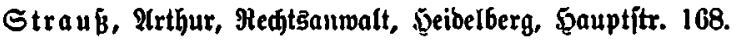

Strefler, Lubwig; Redtsanwalt, Meb̆, Ponceletftr. ?.

Strelib. Medțanmalt, Stettin, Reiffdlägeritr. 4.

Dr. Strobel, (Br. Amṫridter, Rarlsrulje i. $\mathfrak{B}$.

Strof, Juftizrat, Redtsanmalt, Rotar, Glbing, Friebrid Milfelmplał 16.

Dr. Strohal, Emil, Geh. Eofrat, Profeffor, Reipjig, Salomonitr. 1.

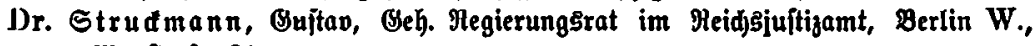
Dopiftrape 31.

Dr. Strudmann, Wirllider Seh. Dber,glegierungstrat, Berlin W., Lanograjen: itrafie 15.

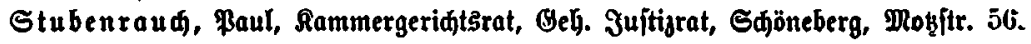

Sturm, Sulius, \&anogeriđtşrat, Raiferstautern, Edjubertitr. 24.

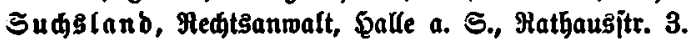

Sud)sland, श(bolf, Ranbgeridttsbixeltor, Torgau.

Dr. Suling, B., Medtsanwalt, Bremen.

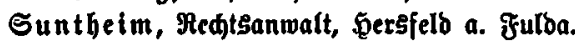

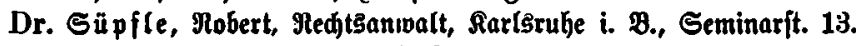

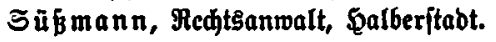

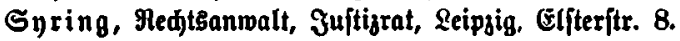

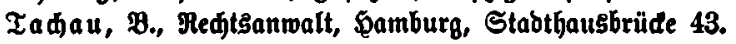

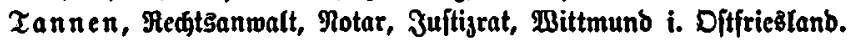

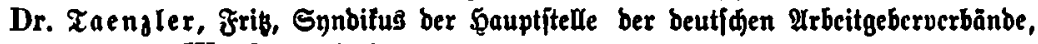

Yerlin W., Sarlsbab $4 a$.

Tappermann, Imtsridjter, Rombad, b. Peł.

Tedow, Mhagijtratsrat, Bertin W., Ban ber Đeydtftr. 7 .

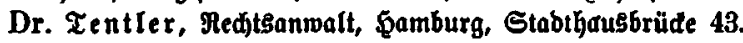

Tefaenborf, Lanbgeridtstat, Berlin W. 30, BerđteSgabenerfit. 38.

Teuberger, Redtsanwalt, Ruhrort.

Teuma

Teutid, Sujtigrat, Res, Steinmes 7.

von Thabben, Wirfl. Albmiralitätsrat unb vortragenber Rat im Meidjmarineamt, Friebenau, 3aitr. 11.

Thal, \&ouis, Brivatbogent, St. Petersburg, Spaj̄taja 16.

Theremin, Sanbgeridtstat a. D., Saweibniţ.

Thiel, Sujtizrat, $\mathfrak{B r e j d e n . ~}$

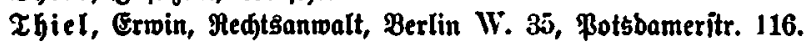

Thiele, Ml., Dberlanbesgeridtsrat, S̈önigsberg i. Pr., Steinbamm 56/57.

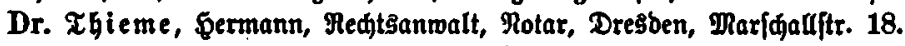

Thiemann, Maz, Medtsanwalt, Braunjdrocig, Bohlweg 66.

Thinius, Milhelm, Rammergeridjtsrat a. D., Bantbirettor, Berlin NW., Calvin: itrafie 14.

Dr. jur. Thie $\lceil$ ing, Imtŝridter, Celle.

Dr. Thoma, Brivatbozent, Freiburg i. P., \&ubwigitr. 36, II.

Thomfen, S., Almtsgeridtşrat, Emben i. Offtrieśtanb.

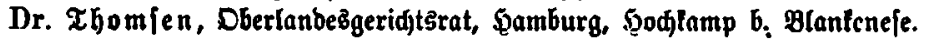

Dr. Thomlen, Suftizrat, Riel, Đoliftenbrüde 4.

Dr. Thomfen, ßrofeffor in ber ređtşs und ftaatsmijienj[aftliden fralultät ber Univerfität Müniter i. $\mathfrak{B}$., Georgscommenbe 1. 


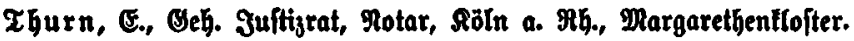

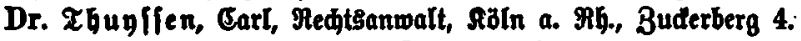

Tiфauer, 3ufthrat, Berlin SW., Bimmer|tr. 61.

Tilleien, Medtsanwalt, Mannheim.

Timenborfer, Mtedtsanwalt, Эuftizrat, Berlin S. 42, Dranienftr. 145/146.

Dr. Titie, Profeffor, Bottingen, Dbere Saripule 35.

Tolltlemit, Mehtsanmalt, Suftizrat, Raumburg a. Saale.

Dr. Toepli

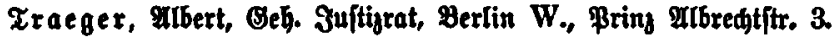

Dr. Traeger, \&., Profeffor, Marburg a. Labn.

Trautnex, \$.. Redtsanwalt, Pamberg.

Dr. Trautwein, Rarl, Medtsanwalt, Pfor gheim.

Treitel, Dberlanbesgeriđt5rat, Röniggberg i. Mr., Zraghenner Pulverftr. 8.

Dr. Trumpler, Snndifug ber நanbelstammer, Franffurt a. $\mathfrak{M}$.

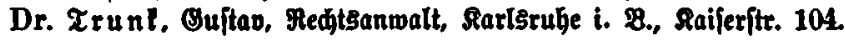

von Tur, Dr. M., Profeflor, Straßburg i. E., Ruppredtsallee 64.

1)r. Tũ rde, Mobert, Lanbridter, Dortmunb, Bismardftr. 14.

Zürt, Medtsanmalt, Juftigrat, Derlin SW., Bimmerftr. 87.

ubles, Emil, Oeh. Suftizrat, Rammergeridtsrat, Berlin W., Tiergartenftr. 3 a.

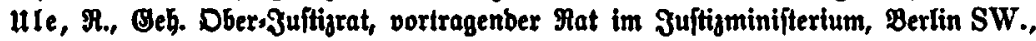
Söniggräberftr. 28.

Dr. von ulImann, Oeh. Rat, MRünden, Rubmigftr. 29,

ultmann, 3uitizrat, Dagbefurg.

ullmann, MedtSanwalt, Rotar. Juftigrat, Pofen.

Dr. Umhauer, Erroin, BeridtEaffefior, Sartsruhe i. Bo., Zenzftr. 12.

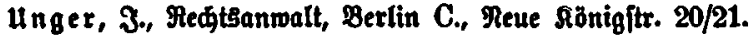

unger, Redtảanwalt, Prenglau.

Biebig, C., 3uftizrat, Berlin, Bülowitr. 24.

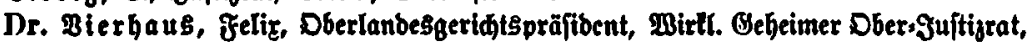
Profeffor, Braslau II, Tauentienftr. 40.

DLeugels, Motar, Juftizrat, Roln, St. Ipernitr. 7.

Dr. Bopel, Medtisanroalt, Maftatt.

Bägele, Mettsanwalt, Rarlšnthe i. 2 .

Dr. פormbaum. Imtstriøter, Berlin W. 50, Bafjaueritr. 38.

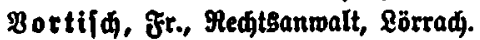

Bo B, 3uftigrat. Birnbaum.

Dr. Boflen, Reo, Medtsanwalt, Düffelborf.

Dr. MaҺ, Beheimrat, Brofeffor, Seipgig. Boethejtr. $\theta$.

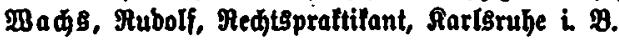

Dr. \$aळtel, Meळtsanwalt, Seipsig.

Dr. Bagentnedt. Fedtsanwalt, Dortmunb.

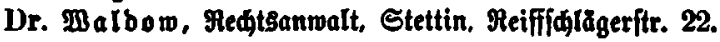

Dr. Malbftein, F.o, Reătsanmalt, Motar, Altona, Rönigftr. 145.

Dr. æa allad, Siegfrieb, Redtsanwalt, ETTen.

Dr. jur. et rer. polit. $\mathfrak{B a}$ aler [tein, Emft, Straßburg i. E.

Dr. Baentig, \$., Brofefior, Walle a. S., Bambergerftr. 31.

Dr. Barburg, Siegfrieb, Redtsanmalt, Juftizrat, UItona a. Elbe, Balmallee 31.

Dr. Marburg, \&. M., Fejerenbar, Gamburg, Ferbinanbftr. 75. 


\section{LIII}

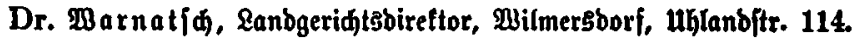

Dr. Warneyer, Dtto, Imtšridter, ReipjigsReubid, Sdmiebftr. 1.

Warfdauer, MedtEanwalt, Motar, Tremefien.

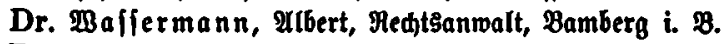

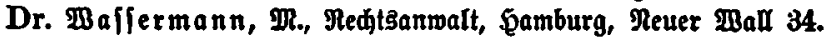

Baffermener, Juftizrat, Bonn.

2Bed, Redtsanwalt, Diebengofen.

2Bebetinb, Beh. Jultijrat, Ranbgerifitsrat, Danjig, Rohlengafie 5.

Dr. 2Bebemeyer, Berner, Bleriatsaffefīor, Bribatbojent, Riel.

Beblan, Reatsanmalt, Notar, Berlin W., Xiergartenjtr. 1.

Behrmann, Redtsanwalt, Notar, Suftigrat, Stettin, Rönigsplak 18.

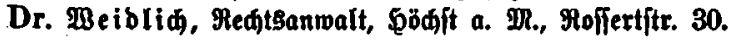

Beibner, פ., Eanogeridtstat, Sempten i. $\mathscr{B}$.

Dr. Beigert, Erid, Medistsanwalt, Cottbus.

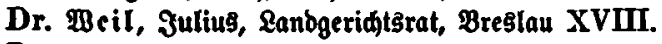

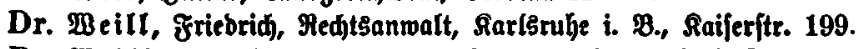

Dr. $\mathfrak{B e i l l , ~ R e o p o l b , ~ \Re e c t t s a n m a l t , ~ R a r I S r u h e ~ i . ~ P . , ~ R a i j e r i t r . ~} 90$.

Wein ber 8 , Medtsanwalt, Berlin C., Rönigítr. 37.

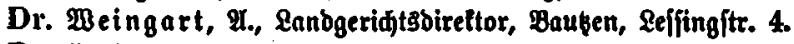

Dr. Weingart. Eugen, Medtsanwalt, Dannbeim.

Beingarten, Medtsanwalt, Rotar, Böpned i. $\mathfrak{x h}$

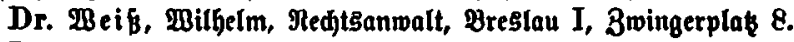

Dr. Mei B, Bearg, Rectsanwalt, Wiesbaben.

Beifer, Th, Reditsanwalt, Seibelberg, Rlofeitr. 17.

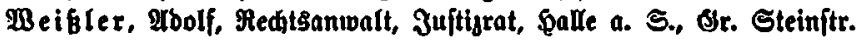

De $\mathfrak{B c l b}$ ige, urban. Medtsanwalt, Paberborn i. $\mathfrak{B}$.

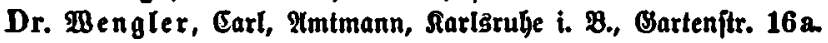

Bens, Medtsanwalt, Zweibrüđen.

Dr. Berner, Joleph, Redtsanwalt, Jultijrat, Bamberg, \$ainftr. 6.

Berner, Emil, Medtsanwalt, Magbeburg, Raiferftr. 13.

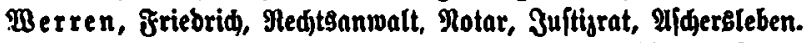

$\mathfrak{B B e r y}$ v. \&imont, Emil, 2 (mtsgeridlstat, Trier, Eberharbitr. 6.

Dr. Werthauer, \$aul, Medtsanwalt a. D., Bel). \$ofrat, Berlin W., Surfïriten= bamm 198.

Dr. Bertheimer, \&ubwig, Redtsanmalt, frantfurt a. Mr., Steinweg 6 .

Dr. Bertheimer, Rectsanwalt, Baben:Baden.

Dr. Bertheimer, Emil, Medtsanwalt, Faiferslautern, Eifenbahnitr. 33.

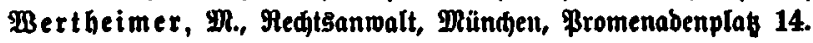

Be ft, Senatsిpräfibent, Sarlïruhe i. $\mathfrak{B}$.

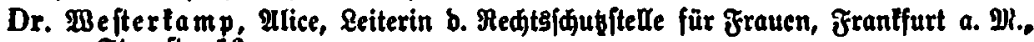
Sternitr. 16.

Dr. Befterlamp, Geh. Sultizrat, Brofeffor, Darburg i. Seffen.

We itp Gal, Oberlanbesgeridtsrat, Samburg 17, Sodjallee 6.

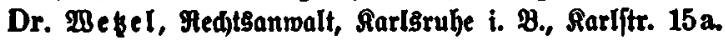

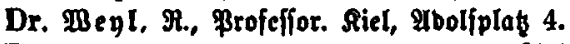

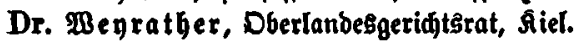

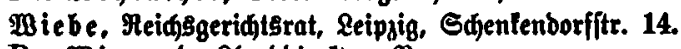

Dr. $\mathfrak{B}$ iegand, 2 loybbireltor, Bremen.

\$ienfowsti, Sammergeridterat, Berlin W. 30, Traunfteineritr. 7. 


\section{IJIV}

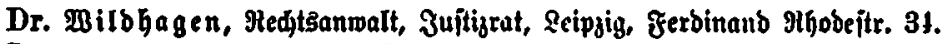

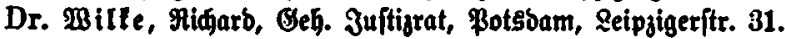

ßilfe, Rarl, Redtsanwalt a. Rammergeridt, Notar, Berlin SW 61, Belleallian cepl. 4.

Bille r5, ఏans, Referenbar, Effen a. RuGr, Senriettenftr. 3.

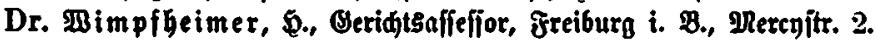

Bintelmann, Friebr., Mchtsannalt, Dortmunb, Eftenhelleweg 3.

Minfler, Ranbgeriatsbirettor, Erfurt, Scrberitr. 31, I.

Bintler, Georg, Redtsanwalt, Notar, Eangenjalza.

Bintler, Meditsanwalt, Maing.

Wintler, Oberamtšridter, Sarlšruhe i. $\mathfrak{B}$.

Binter, E., Pmtmann, Sarlsrulje i. B., Borholzitr. 48.

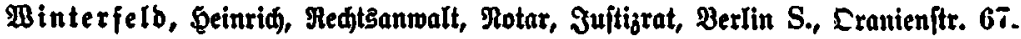

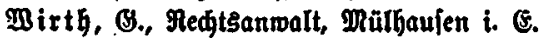

Bitte, Lanogeriditspräfibent, Beh. Dberşuftiłrat, Düffelborf.

Dr. Wittelshö fer, Medtsanwalt, 3uftizrat, fourth in Bayern.

Dr. Wittern, Medtsanmalt, Riel.

Dr. Wittlo ws $\mathbf{f y}$. Fledtsanwalt, Berlin W., von ber פeybtitr. 7.

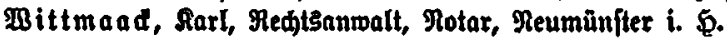

Dr. Wittmer, Zelix, Red)tŝnnwalt, Mannbeim.

Bize, Redtsanwalt, Riffa.

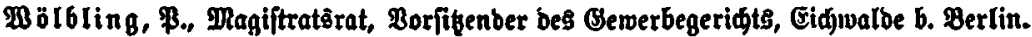

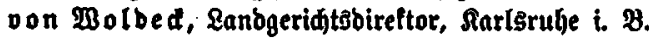

Dr. $\mathbb{B}$ olf, Ebuarb, 3uftizrat, Meditsanwalt u. Motar, Dresben, 3obann Beorgen= elfee 5, II.

Wolfen, Juftizrat, Reditsanwalt, Rotar, Bromberg, Milhelmitr. 13.

Bolff, Emit, arfefior, Berlin W. 30, Bayeri/der Plat 2.

Dr. Molff, Drartin, Profeffor, Derlin W., Rütorittr. 66.

Wolff, Frand, Medtitsanwalt, Notar, Duulbein a. Muhr.

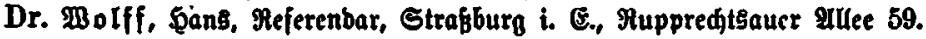

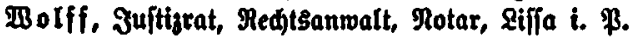

Dr, Molff, Midharb, Medtsanwalt, Suftizrat, Berlin W., Erjaperftr. 10.

Dr. Bolff, Mag, Medtsanwalt, Rifia i. $\$$.

Dr. Molff, Medtęanwalt, Berlin NW., ltnter ben Sinben 60 .

Bolff, Eugen, Nedtsanwalt, Juftizrat, Berlin W., Bülowitr. 85.

Bolff, Curt, Medteanwalt, Berlin SW., Zriebrichitr. 243.

Bolf ram, Emil, Geh. Megierungstat, vortragenber Mat in ber Eberredgnungs: Eammer \$otsbam, Gijenhartift. 8.

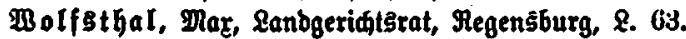

Dr. Bollf, R., Redtsanwalt, Rarisruhe i. $\mathfrak{B}$.

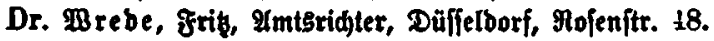

Brefdnet, Semi, Medtsanwalt, Berlin N. 24, Dranienburgeritr. 33.

Wrefđner, \&ubw., Suftizrat, Berlin W. 66, 2Bilfelmftr. 55.

Bronfer, Mechtsanwalt, Suftizrat, Berlin C., Saifer \$illhelmiftr. 49.

Bulff, Baul, Rechtsanwalt, Berlin S., Dranienftr. 130.

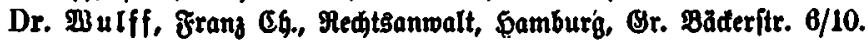

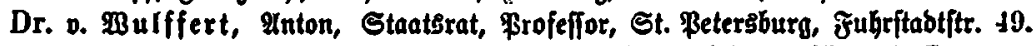

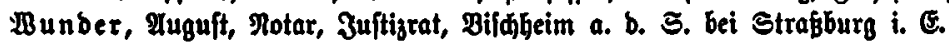

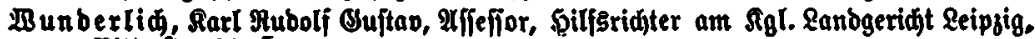
Milterftr. 21. I. 


\section{LV}

Bunberliđ, Medtsanwalt, Dppeln.

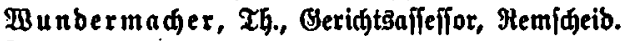

Dr. Büiteriö̈rfer, Seriditsafjefior, Brivatbojent an ber Ganbelshodjidule Cöln-

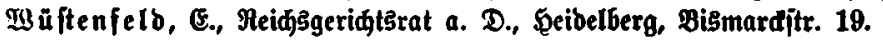

?) $l$ in, Staatsanwalt, Stuttgart, Reue Weinjteige 12.

Dr. Babler, Bruno, Redtsanwalt, Baben=Baben.

3adariae, Dtto, Senatspräfibent, Berlin W., Baffauerftr. 11 a.

3ander, Nedtsanwalt, Notar, Erjurt, Neuwerfftr. 3.

Bborowsti, 2rmtsgeridterat, Sobenjalza.

Dr. Behme, Feliz, Medtanmalt, Reipzig, Sđjillerjtr. 3.

Dr. Bei $\bar{B}, \mathfrak{S} .$, Juftizrat, Jena.

Dr. Beller, ఏeintid, Darm[tabt, פodjftr. 66. II.

Belter, Meditzanmalt, Jujtigrat, Stettin, 2ugujtaplą 1.

Dr. Benner, Medtenanwalt, Straßburg i. E.

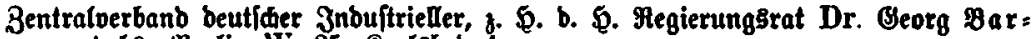
tels, Berlin W. 35, Sarlsbab $4 a$.

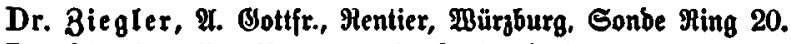

Dr. Biegler, Br., Medtsanwalt, Rartsruhe $i$ g.

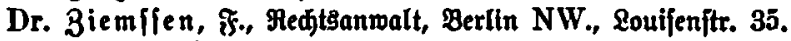

Dr. Biefe, Réditsanwalt, Suitizrat. Menbsburg.

Bimmermann, Oeh. Finanjat, Sartsrube i. $\$$

3inbel, Minifterialbirettor, Stuttgart.

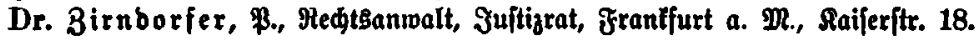

Dr. Bitelmann, Beh. Sujtizrat, Brofefior, Bonn, Eoblenzeritr. 83.

Boeller, Sulius, Ritter von, Ober[taaţanwalt, Münden, Pring Rubmig[tr. 16, II.

Bopff, Carl, Beigeorbneter, Dffenbad).

Dr. Born, (\$eh). 3uftizrat, Brofeffor, Bonn.

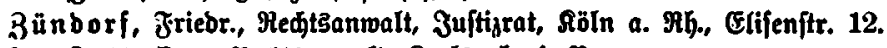

Dr. Butt. Dtto, Redjtsanmalt, Rartšrube i. $\mathfrak{B}$.

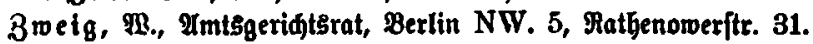

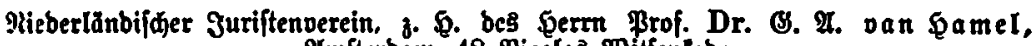
Utmiterbam, 48 Ricolaş 2 itjenfabe.

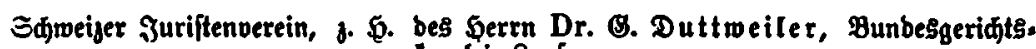
tanglei, \&aulanne.

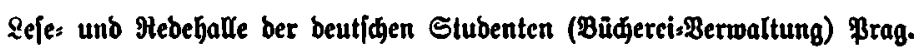

Internationaler $\mathfrak{A} n$ malts:Berband, Wien I, Bonzagagaffe 16.

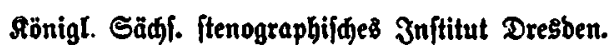

Bibliothet ber Deputation bes Deutiden Juriftentages, Berlin W. 35 , PotBbamer: ftrafe 118.

Correfponbenz פerzberg, Berlin SW. 48. Endeplak 3.

Sönigl. Bibliothet Berlin.

Univerfitätsbibliothet Berlin. 


\section{Berzeidnis \\ ber

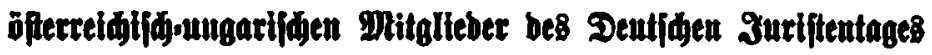 \\ im Jabre 1908.}

Dr. Ibel, ßaul, \$of; unb Oeridtsabolat, \$ien I, Mofenburjengafie 8.

Dr. Ibler, Em., Brivatbogent, \$ien VII, Ranblgaffe 36.

Dr. Ibler, Sarl, thiverfitätsprofeffor, Ejernomib.

Dr. Mbler, Mbolf, ఏofs unb Oeridtsabvolat, Mien I, Braben 12.

Dr. Mbler, Siegmumb, Univerfitätsprofeffor, \$ien VII, Zeelbgafje 10.

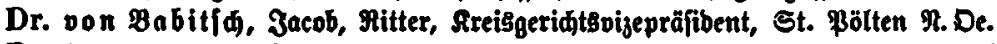

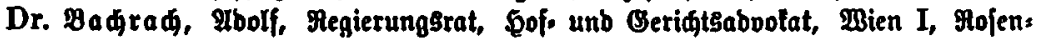
burfenftr. 8.

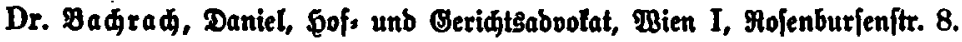

Baltinefter, 3ołannes, t. t. Notar, \$ien XVI, Ottatringerftr. 3!.

Dr. Baernreither, 3ofef, Dinifter a. D., Mien, Sanogeriftsftr. 11.

Baum, Th., Derausgeber ber Spars unb Mentenjeitung, MBien I, Mipplingerftr. 5 .

Dr. Benba, Cbuarb, Ibootat, Xeplit, Sdoenau, Rangegafie 41.

Dr. Denebict, Ebmunb, জof. und Beridtsabootat, \$ien I, Sdjottenring 19.

Bertbolb, Carl, |alef. Ranbesfetretär, Borftanb beS Lanbes[tatififiden Ifmts, Xroppau.

Dr. Beurle, Carl, Itboblat, \&inj, \&anbitr. 9.

Dr. BIa as, Đermann, t. t. Fuslultant, Gurl i. Saerntben.

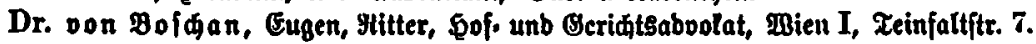

Dr. Bo Bcovib, 2rffreb, ฐof, unb Beriatsabodat, Mien I, Boltsgartenftr. 1.

Dr. von Bogoti, Mlogs, RgL. Mat, Direltor ber Rgl. Medtsalabemie, Magn Barab.

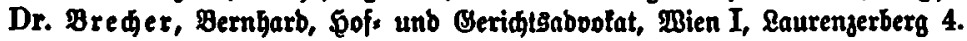

Brielmanex, IIffreb, t. t. Ranbesgeridtsrat, פloebling, Biedtlgaffe 4.

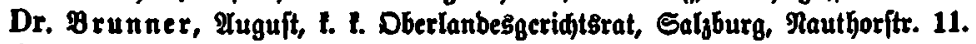

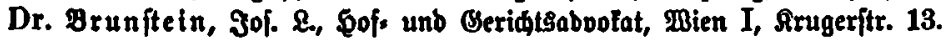

Dr. Byloff, Zriß, Bribatbojent, Braz, Ridjard æagnergafie 8.

Dr. von Call, Zriebrid, Zreigerr, t. t. Dberlanbeggeridgtspräfibent, Exgelleng, Snnebrud, QYniditt. 42.

Dr. Coumont, Ebuarb, Đof; und Geridtsabvolat, Wien I, \$alfifdgafie 4.

Dr. Dalnofy, Bela, Medtsanwalt, Medtstonfulent bes Sanbeis:Breniums, Buba= peft $V$, seopolbring 8.

Daunjer, Carl, Dberöfterr. Ranbegrat, utrabr b. Linz, Sauptfitr. 75.

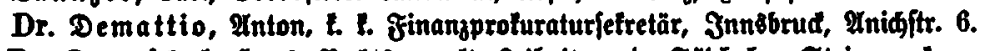

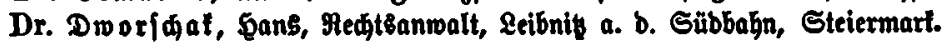




\section{LVII}

Dr. Ehrengweig, Ilrmin, Brofefior ber Medte, Bien VIII, Laubongaffe 37.

Dr. Eifenberger, Bilhelm, Ilodolat, Starisbab.

Dr. Ergner, \&eo, t. t. ફofrat bes oberften Beriats. u. Saffationghofes, खien IX 4, Bürtel 118.

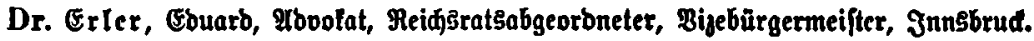

Dr. Ettinger, Dlarcus, \ofs unb (Seridtsabootat, Wien VII, Dlariabilfftr. 8.

Dr. Farnil, Zgnas, 2boofat, Sdivab, Tirol.

Dr. Feigl, Dstar, 5ofs und Beridtsabnofat, Wien I, Salvatorgaffe 6.

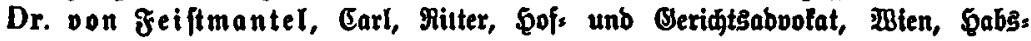
burgergafie 5.

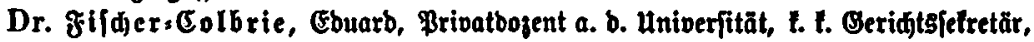
Wien I, RohImartt 11.

3. u. Dr. Fleifdmann, Carl, Ilboolat, Rarlsbab.

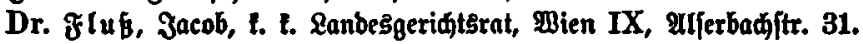

Dr. Frantl, Dtto, univerfitätsprofeffor, Brag. Smidfo, Ferbinanbstai 16.

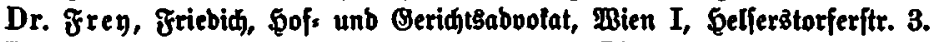

Dr. Frieb, alfreb, Itboolatursfanbibat, wien VII, Sumpenborferftr. 12.

Dr. Frił, Ernit, t. t. Babnfommifjar, Jnnsbrud, Graltftr. 9.

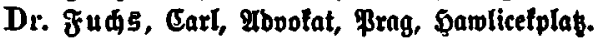

Dr. Gabl, Sans, Ilboolat, \&anbed, Tirol.

Dr. Ballia, Mbolf, \$ofs unb Beridtsaboolat, Mien I, Biberitr. 2.

Dr. Oallia, Lubrig, Mboolat, \$ien I, Biberitr. 2.

Dr. Bellex, Leo, Sofs unb Beridtsabvofat. Mien I, Fran Jofefs Duai 25.

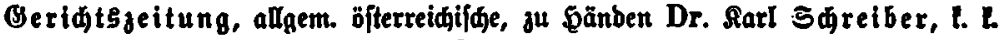

Settionsrat im Eijenbagnminifterium, Mien.

Dr. Beringer, Bictor, f. t. Finangrat, Grag, Refingftr. 8.

Dr. (Sert [d)er, Mbalbert, Dberlanbeşgeriditspräfibent, Trieft.

Biegl, Julius, Dberlanbesgeridtstat, Bien XVIII 1, Eane Sadjgafje 3.

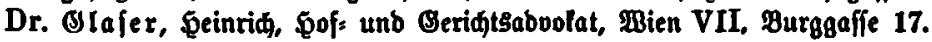

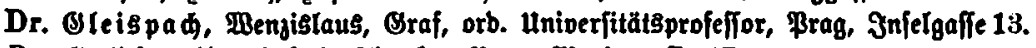

Dr. Bolbfamibt, alfreb, Qtovotat, Brag, Mariengaffe 17.

Dr. Graf, פeinriđ, Đofs unb Geridtsaboolat, Wien I, Werberthorgafie 4.

Dr. jur. \&rae $\sharp$, Bictor, \$ien I, Sanjenftr. 6.

Dr. Oreistorfer, Garl, Mrovotat, Sraj.

Dr. Grof, Siegfrieb, Sofs unb Geridtsaboofat, Mien I, Sduultergafie 5.

Dr. (S) ränberg, Univerfitätsprofeffor, æien XVIIl, Benßgaffe 38.

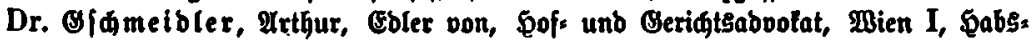
burgerftr. 6.

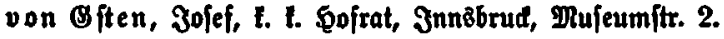

3. u. Dr. SaGn, Carl, Ilbootat, Sarisbab.

Dr. \$amm, Catl, t. t. Tajidjstidter, Nonbiee.

Dr. Saemmerle, Dtto, Iiboofat, Sufftein, Tirol.

Dr. \$aerbtl, \$cinrid, Jreiherr, Mien I, Stallburggaffe 4.

Dr. Sanaujef, Guftav, a. o. Univerfitäteprofeffor, Braz, Elijabethjtr. 20.

Ganbels: unb Bewerbetammer Eger.

Dr. von Gattingberg, Jolef, Mitter, f. t. Megierungstat, Salzburg, Sarolinens plaz 3.

Dr. von Sernritt, Profeffor, Wient. 
Dr. Gilber, Sieronymus, 9lbotat, Pruned, Tirol.

Dr. \$lavàc, Ybolf von, Ebler vom Meditwall, t. t. Finanjproluraturielretär, Bien VIII, Budjelogaffe 4.

Dr. \$öfler, Hubolf, Geriatsabjuntt, Reoben, Saernthen.

Dr. Eocgel, Dugo, t. Dberitaatsanmalt, Profefior an ber Sonjularatabemie, Wien VII, Meujtiftgaffe 17.

Dr. Sobenburger, Bictor, $\Re$. v., Rehtsanwalt, Bra\}, Fif

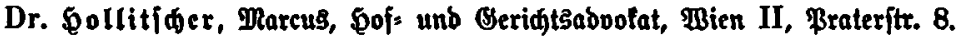

Dr. Soenig, \&riebrid,. Beneralfetretär b. Beamtenvereins, Mien I, Mipplinger: itrabe 25.

1)r. von Goermann von Goerbad, M., Profefior, Rettor ber univerfität Зñ⿰纟brud.

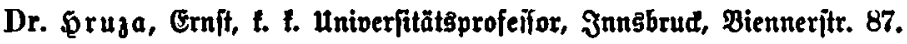

Dr. Sugelmann; Carl, t. t. \$ofrat, פien XIX, Chimanbftr. 19.

Dr. Supla, 3olef, ærofeffor, \$ien VIII, Ebelhofgaffe 36.

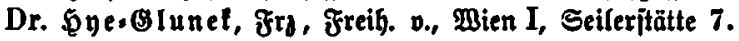

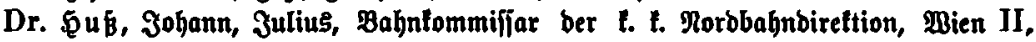
Iugbadjtr. 14.

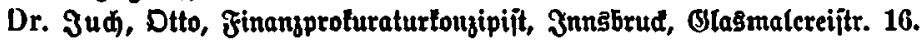

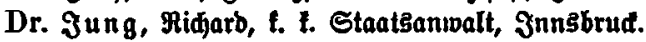

Dr. Эunfar, Emil, t. t. öfterr.sungar. Bizetonjul, æBien I, Ballfausplak 2. 3uriftifde Oelellfdaft, Troppau.

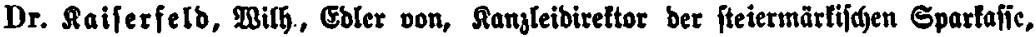
Oraz, Sdqmiebegaffe 2.

Dr. Raiferfelb, Mar, Fitter v., Ilboolst, Oraz, Sporgaffe 32.

Dr. Sammerlanber, Subwig, \$ofs unb Geridtsaboptat, Sraj, Slacisjitr. 27.

3. u. Dr. Raufmann, Jriebrid, Ylobotat, Prag, Sraben 17.

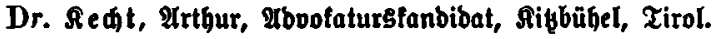

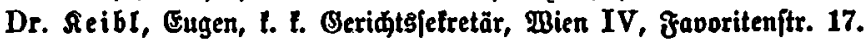

Dr. RIang, Эames, Berwaltungšrat bes öfterreid). ßhöniz, Wien I, Mltemergafie 2.

Dr. Alein, Franz, Ezjell., Juftizminifter a. D.. t. I. Beh. Flat, Seltionsdef uno Univerfitätş=\$rofeffor, æien VI, Oumpenborferitr. 5.

Dr. Rlima, Unton, Ybootat, Gra\}, Raijerfelbgaffe 1.

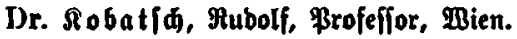

Dr. Rolbe, Dominif, \$of. und Seridtsabodtat, Mien IV, 2uleegaffe 28.

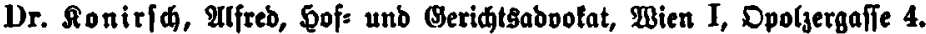

Dr. Pörnyei, Ebuarb, Rbootat, tönigl. Hat, Bubapeit IV, Deát:uła 5.

Dr. Rofda der, Paul, Brivatbojent, Oraf, Riebiggaffe 22.

Dr. Sowarz, Bilhelm, t. t. Babntommifär, Ling, Rarjiftr. $15 a$.

Arapf, Carl, t. t. Sanbeşgeridtsrat, Graz, Sumbolbitr. 21.

Dr. Ara\{a, Bertholb, ఏofs und Beriđtsabootat, Bien I, \$elferstorferftr. 13.

Dr. Arasny, Arnolb, f. t. Seltionsrat, Brofeffor a. b. Sonfularatabemie, Mien I, Elifabethitr. 9.

Dr. Rraus, Carl, Medtsanwalt, Teplił i. Böhmen.

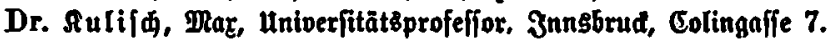

Dr. Sunwalb, \&ubw., \$of z und Beridtsabvotat, Bien I, Sdaleritr. 1.

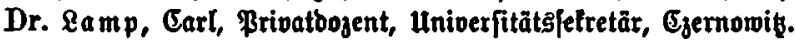


1)r. Lanbauer, Wallher, Direftionsfefretär ber Witfomizer Bergbau= unb Gifen=

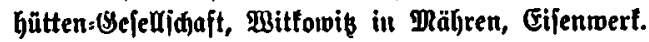

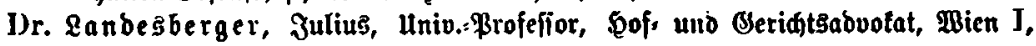
Sdottenring 17.

\&anbeşverband ber ungar. Gifenwerte und Mafdincnjabrilen, 8. 5. bes Gerrn Minifterialrat von Foeriter, Bubapeit 190, V, Thonetuboar.

Dr. \&anger, Эofef, நof: und Geridjtaboolat, Wien I, Bäđer[tr. 9.

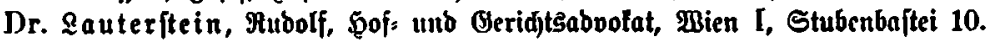

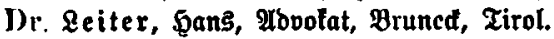

3. u. Dr. Lent, Bittor, t. t. Notar, Jriebland i. Wöhmen.

Dr. \&ens, $\mathfrak{A}$., Univerfittätsprofeffor. Wien XVIII, Sduulgafie 36.

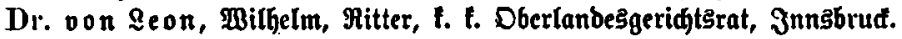

Dr. Qévy Béla, Meditsanwalt, Bubapeít V, Balvampęa 5.

Dr. \&idt, Stefan, Mcidstat3abgcorbneter, Mien.

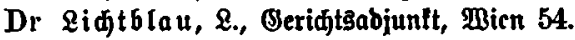

Dr. Linfs, Emil, Sof= und Beridtsabodat, Bien I, \$ohenitaufengafie 2.

Dr. \&öffler, Allecanber, Univerfitätsprofeffor, Mien XVIII, Senłgafíe 38.

D1. Eobling, Ernit, Ibootaturgtonjipient, Wien IX 3, Edjmarjfpanierftr. 12.

Э. u. Dr. \&öw, Buftav, Ilbovolat, Mähr.:S丸̈̈nberg.

Dr. Eoewenthal, Dagobert, Fabrifant, Iglau.

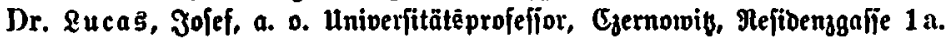

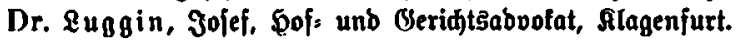

Dr. \&u[din von Ebengreuth, Arnolb, פofrat, Itniverfitätsprofeffor, Gra\}, Perangafie 15.

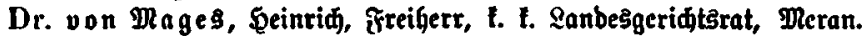

Dr. פRaier, Gugo, ఏofs unb Geridjtsabootat, Wien I, STugeritr. 13.

Dr. von $\mathfrak{P l a f}$ owi , Ebuard, Geriđtsfetretär, Wien I, Juftijminifterium.

Mardjefani, Anton, t. t. Lanbeşgeridjt3rat, Sd)runs, Borariberg.

Dr. DRa rgulics, Emil, IlobolaturStonjipient, Trautenau.

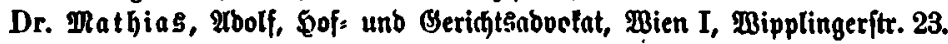

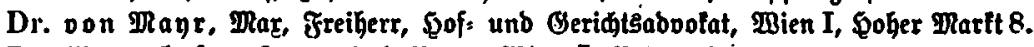

Dr. MRayerদ̆ ofer, Franz, t. t. Motar, Wien I, Betersplał 7.

Dr. Menger, Mlax, \$ofs unb Geridtsabootat, Wien VIII, Ruerspergitr. 19.

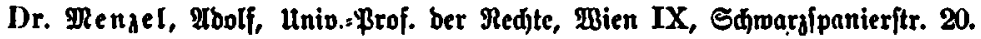

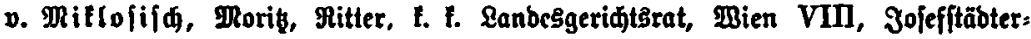
ftrafie 11.

Dr. PRorgenftern, פugo, פof= unib Beridtsaboofat, Wien VI, Mariahilferftr. 127.

Dr. Diüller, Milfhelm, Ranbestrat, Rlagenfurth i. Saernthen, Lanbhaus.

Dr. Nedjansty, Ruguft, Sof: unb Oeridtsabuotat, Wien I, Stop im Simmel 3.

Dr. Nitter von Neumann:Ettenteid, Mobert, Mat bes t. f. Berwaltungs: geriatșhofes, æBien III, ungargafie 16.

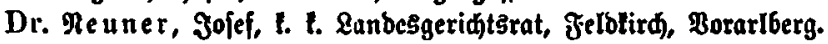

3. U. Dr. Meußser, Sugo, Medttanmalt, Troppau.

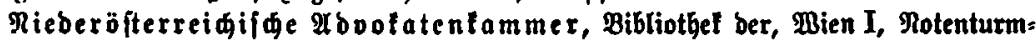
ftrafe 15.

Dr. Dberfogn, friebrid, §of. unb Gerid)tsabvotat, Mien I, ffeifdmarlt 18.

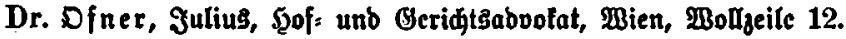

Dueftinghel, Cổar, t. t. Bejirfäridgter, Sillian, Tirol. 


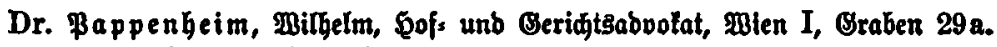
Batrnn, Rubrig, LanbeSregierungšrat, Troppau.

Dr. Peer, Jofef, Irooolat, Zelbtixh, Borarlberg.

Dr. PerImann, Walter, Yrbootat, Brag, Braben 10.

Dr. \$faff, \&eopolb, ‡. z. Univerfttätsprofefior, खien III, Erbbergftr. 9.

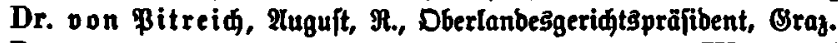

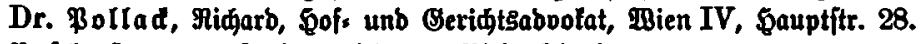

Bofd, Sermann, Eanbeggeridjtgrat, Nieb, Tirol.

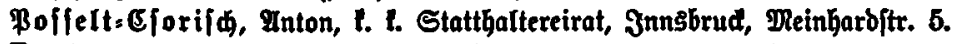

Dr. Breminger, $\mathfrak{M}$., t. t. Beridjtsabjuntt, Storosnnebs, Bulowina.

Dr. Pibram, Rarl, Ilovotaturstanbibat, \$rag. Oraben 10.

Brobinger, Guftav, t. t. Beridtsabjuntt, Begau, Boralberg.

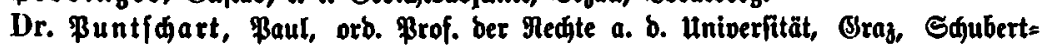
ftrafe $19 \mathrm{II}$.

Dr. Buß̨er, Enil, Mlovolat, Brünn.

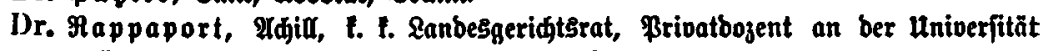
Gjernowif i. Butowina, Zrangensgaffe 39.

Dr. Mebenfaft, פbolf, \$of: und Beridtşabvofat, \$ien I, Sdüler[tr. 22.

Dr. Mebliq. 3ojef, Brofefior, Mien IX, Irrmbrujtergafie 15.

Dr. Meblid, நarry, Mien IX, Beregringaffe 2.

Dr. Meid, Max, 1. t. Motar, Seoben i. Stcicrmarl.

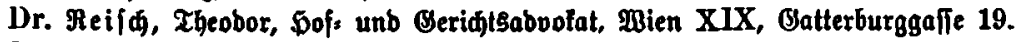

Dr. Neitmaner, Ilfreb, Beriats:2ustultant, Sufftein.

Dr. Meyer, Emil, em. Ilovolat, Trieft, via Vienna 1.

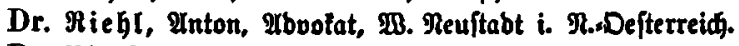

Dr. Riet\{d, Rarl, t. t. Rotar, Reuern i. Böbmen.

Dr. Mintelen, Inton, Brofeffor an ber beutífen univerfität \$rag.

Dr. von Fotitansti, Carl, Freiherr, t. t. Beriatsabjuntt, 2luffee (Steiermart).

Dr. Folfer, 3ulius, t. t. Lanbgeridtsrat, \$ohenelbe i. Pöbmen.

Dr. Mofenbader, Mrnholb, 2lbootat, Brag, Beltnergaffe 2.

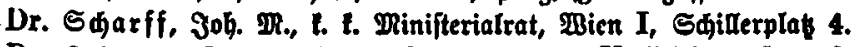

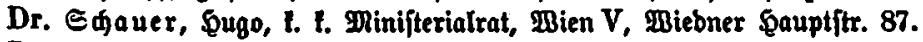

Dr. Saellanber, Franj, 2rbootat, Trieit, Sorjo 35.

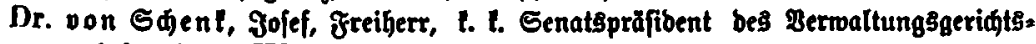
Gofes, פBien III, Sauptgaffe 75.

Dr. oon Sdey, 3ojef, Baron, Brofeffor, Wien IV, Iherefianumgafie $2 a$.

Dr. Sqiffner, \$rofeffor, Đofrat, Jnngbrud.

Dr. SdIoffer, Mlois, Bräfibent ber Qbootatentammex, Sraj.

Dr. Sđueeberger, \$ilbelm, 5ofs und Beridtsabvolat, \$ien I, Maria Therefien= ftrabe 10.

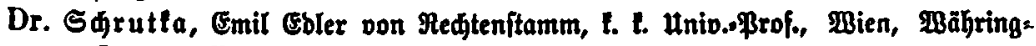
Eottagegaffe 53.

Sduler, Ferbinand, Bejirtsinidter, Nanbers, Tirol.

Dr. Eduloff, Theodor, §of: und Beridtsaboolat, Mien J, Shellinggaffe 6.

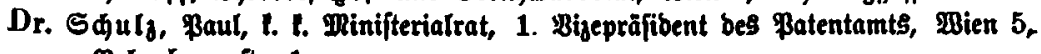
Babenbergeritr. 1.

von Sqwarjenau, Erwin, Freiberr, t. 1. Stattbalter, Erzelleng, 3nnsbrud, PurgDr. von Sdyard, Georg Ebler von, Brag II, f̧ügnerplał 5. 


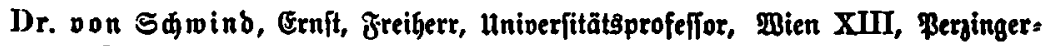
itrabe 66.

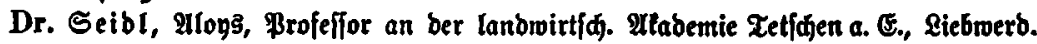

Dr. Seibler, Suftav, f. t. Unio.= Brofeffor, Bien IX, Safenaueritr. 53.

Dr. Spiegel, \&ubmig, univ.=\$rofeffor, Brag II, Znregaffe 7.

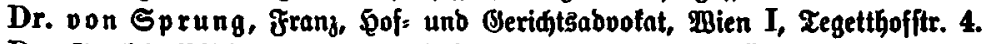

Dr. Stahl, \$hilipp Mitter v., t. t. Settionsrat, Wien IV, Rarlsgaffe 13.

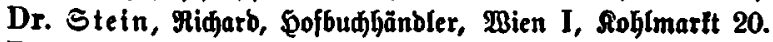

Dr. Ste inbad, Gujtav, Mebatteur ber Meuen freien \$reffe, Mien I, gidtegaffe 11.

Dr. Stcinleđner, \$aul, t. t. \$ofrat, o. ̈. Univerfitätsprofeffor, \&raz, \&lacis= jtrafe 57.

Dr. Sternberg, Dtorik, Berteibiger in Straffadjen, Mien I, Stubenring 14.

Dr. von Stourab, Alois Ebler, t. t. Gofrat, Bizepräjibent bes t. t. Streisgeridts. Rornenburg bei Wien, Sdaumanngafie 12.

Dr. Strangly, פeinrid, ఏof: und Beridtsabvolat, \$ien I, Gonzagagaffe 12.

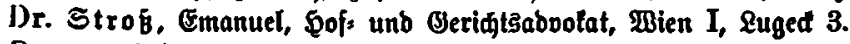

Dr. von Tárnoçsy Sprinjenberg, Ehriftian, Mitter, t. 1. Iusfultant, Eras, Burggaffe 11.

Dr. Teffabri, Pius, Oeridtsabjuntt, Trient, Lanbesgeriđt.

Dr. Thanner, Friebrid, Univerfitätşprofeffor, Oras, Baalsftr. 8.

Dr. von Thayenthal, Mac, Setretäritelloertreter ber Biener Sanbels: unb Bes werbelammer, Wien I, Mipplingerftr. 34.

Theumer, \&eo, t. t. emer. Rotar, Elbogen.

Theumer, Emil, t. t. Motar, Elbogen.

Dr. Thumim, Nathaniel, £ofs und Geriđtsaboolat, Wien I, Subenplał 8.

Dr. Torggler, Sans, t. t. Finanzrat, Jnngbrud.

Dr. Traunfte iner, 3obann, Mboolat, אiłbübel, Tiror.

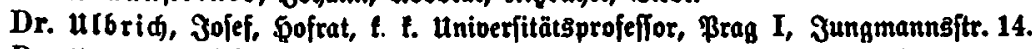

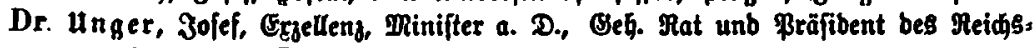
geridts, wien I, Santgaffe 3.

Dr. Hranitjø, Emil, Ilboolat, Graj, Raijerfelbgaffe 21.

3. u. Dr. Bod, Frany, t. t. Motar, Raibad i. §rain.

Dr. von Boltelini, ૬ans, o. ö. Univerfitätకprofeffor, Эnnsbrud, Inidiftr. 18.

Dr. Bagner, Sarl, t. t. Potar, Wien I, Tiefer Graben $\theta$.

Dr. Balfer, Gujtav, t. t. Geriats\}etretär und Đrofeffor an ber Univerfität Jnns: brud, Dharia Therefienftr. 36.

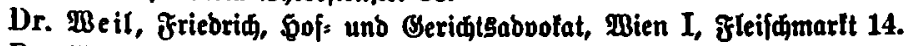

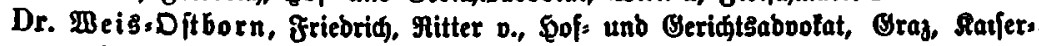
felbgafie 22.

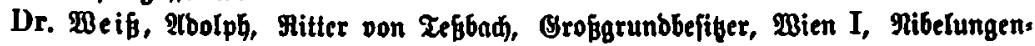
gaffe 1.

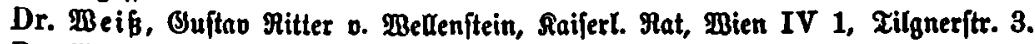

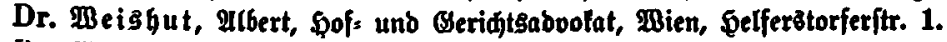

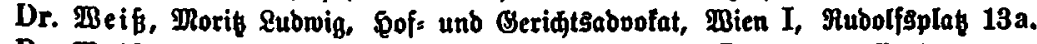

Dr. Beifel, Edmunb, \$of= und Seridtsabofat, \$ien I, Straudgafle 1.

Dr. Wellfpader, Morik, univerfitätsprofeffor, Wien IX, Majagafje 50.

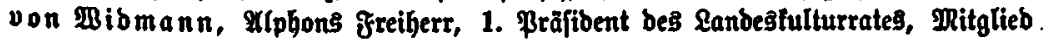

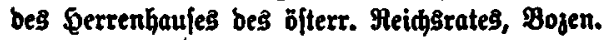


Dr. Mien, frand, Mboolat, Rrag, \$engelsplał 7.

Dr. von Winiwarter, Frang Mitter, Gof; und Geridtabootat, Wien I, Dom= galie 6.

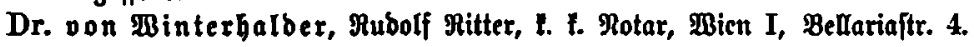

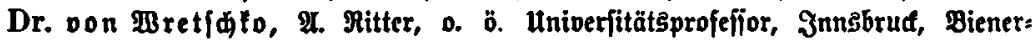
ftraje 27.

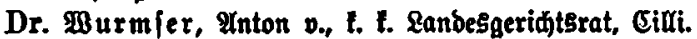

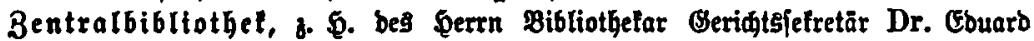
filder:Colbrie, \$ien, Jujtizpalajt.

Dr. 3oblow, Midael, Privatbojent und Seridjtefetretär, Sofia. 